Key Words: Apatite, Zero-Valent Iron, Stabilization, Sediment, Contaminants, Zone-of-Influence

Retention: Permanent

\title{
Phosphate Mineral Source Evaluation and Zone-of-Influence Estimates for Sediment Contaminant Amendments at the TNX Outfall Delta Operable Unit (U)
}

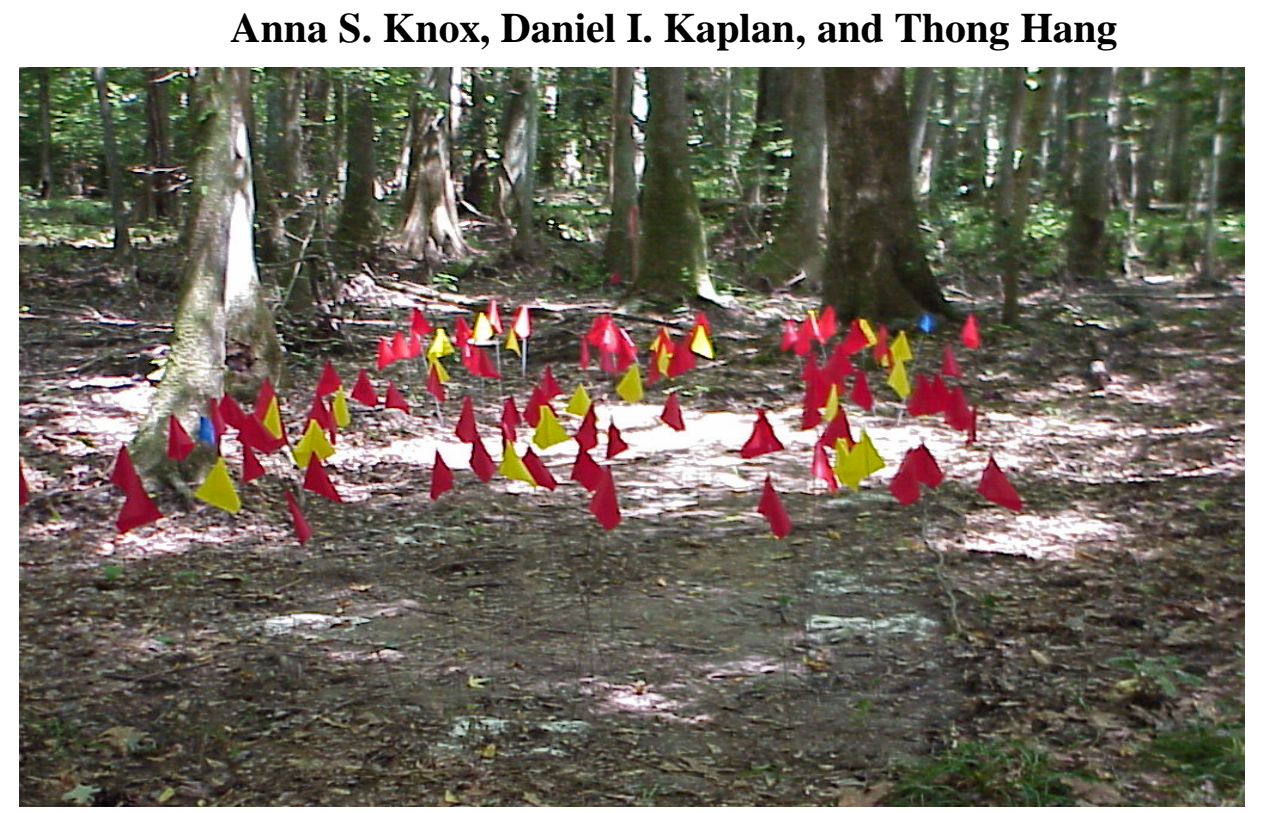

December 29, 2003

Westinghouse Savannah River Company

Savannah River Site

Aiken, SC 29808

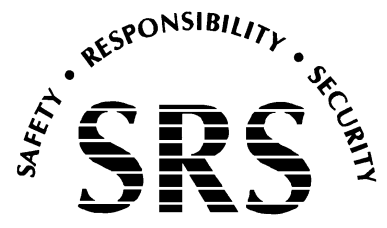

SAVANNAH RIVER SITE

Prepared for the U.S. Department of Energy Under

Contract Number DE-AC09-96SR18500 
This document was prepared in conjunction with work accomplished under Contract No. DE-AC09-96SR18500 with the U. S. Department of Energy.

\section{DISCLAIMER}

This report was prepared as an account of work sponsored by an agency of the United States Government. Neither the United States Government nor any agency thereof, nor any of their employees, makes any warranty, express or implied, or assumes any legal liability or responsibility for the accuracy, completeness, or usefulness of any information, apparatus, product or process disclosed, or represents that its use would not infringe privately owned rights. Reference herein to any specific commercial product, process or service by trade name, trademark, manufacturer, or otherwise does not necessarily constitute or imply its endorsement, recommendation, or favoring by the United States Government or any agency thereof. The views and opinions of authors expressed herein do not necessarily state or reflect those of the United States Government or any agency thereof.

This report has been reproduced directly from the best available copy.

Available for sale to the public, in paper, from: U.S. Department of Commerce, National Technical Information Service, 5285 Port Royal Road, Springfield, VA 22161, phone: (800) 553-6847, fax: (703) 605-6900

email: orders@ntis.fedworld.gov

online ordering: http://www.ntis.gov/help/index.asp

Available electronically at http://www.osti.gov/bridge

Available for a processing fee to U.S. Department of Energy and its contractors, in paper, from: U.S. Department of Energy, Office of Scientific and Technical Information, P.O. Box 62, Oak Ridge, TN 37831-0062,

phone: (865)576-8401,

fax: (865)576-5728

email: $\underline{\text { reports@ adonis.osti.gov }}$ 


\title{
Phosphate Mineral Source Evaluation and Zone-of-Influence Estimates for Sediment Contaminant Amendments at the TNX Outfall Delta Operable Unit (U)
}

\author{
Anna S. Knox, Daniel I. Kaplan, and Thong Hang
}

December 29, 2003

Westinghouse Savannah River Company

Savannah River Site

Aiken, SC 29808

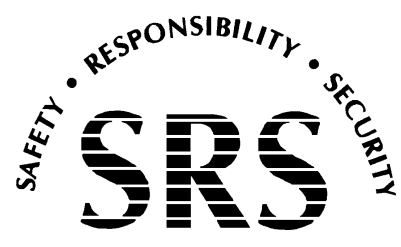

SAVANNAH RIVER SITE

Prepared for the U.S. Department of Energy Under

Contract Number DE-AC09-96SR18500 
This page was intentionally left blank 


\section{TABLE OF CONTENTS}

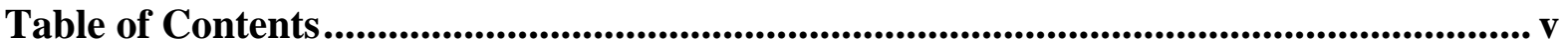

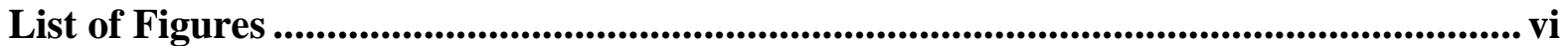

List of Tables.................................................................................................................. vi

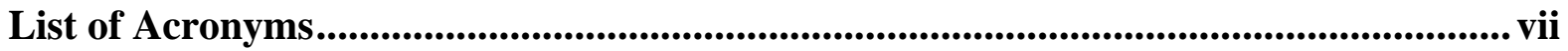

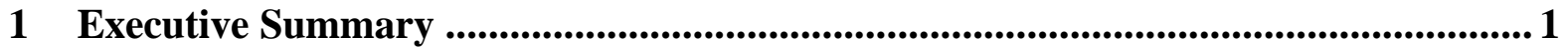

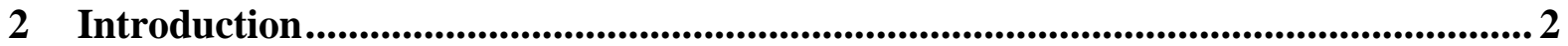

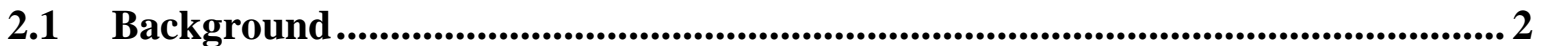

2.2 Immobilization of Contaminants by Phosphate and $\mathrm{Fe}(0) \ldots \ldots \ldots \ldots \ldots \ldots \ldots \ldots \ldots \ldots . . . . . . . .22$

2.3 Phosphate Rock - Phosphorous Sources........................................................... 3

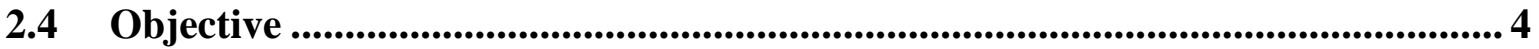

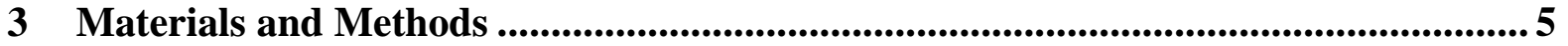

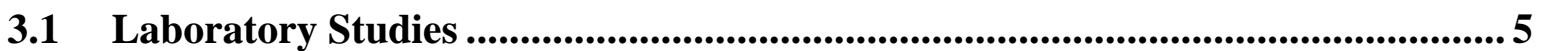

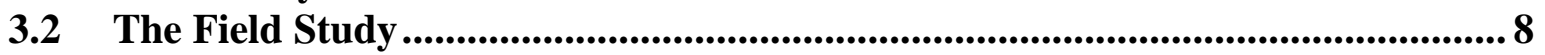

3.3 Sample Analysis and Quality Assurance......................................................... 11

4 Results and Disccusion ................................................................................. 11

4.1 Phosphate Material Characterization ............................................................ 11

4.2 Effective Diffusion Coefficients Calculated from Kd Values ............................... 20

4.3 Zone-Of-Influence (ZOI) of Amendments Under Laboratory Conditions......... 22

4.4 Zone-Of-Influence (ZOI) of Amendments Under Field Conditions................... 25

4.5 Phosphorus and Iron Diffusion Modeling...................................................... 29

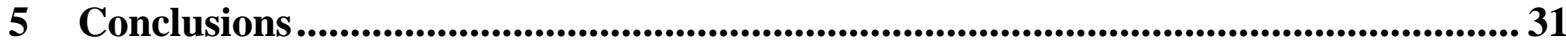

6 Acknowledgements .......................................................................................... 31

7 References............................................................................................................ 32

Appendix A: Additional Data from the Laboratory Studies..............................35

Appendix B: Work Instructions for the Laboratory Studies...............................40

Appendix C: Work Instruction and Additional Results from the Field Study..............52 
WSRC-TR-2003-00579, REV. 0

\section{LIST OF FIGURES}

Figure 1. Experimental Design of the Field Study of Zone-of-Influence (ZOI) at the TNX OD

Figure 2. The Distribution Coefficients $\left(\mathrm{K}_{\mathrm{d}}\right)$ for $\mathrm{P}$ and Fe for the Sediment (B5) from the TNX OD 20

Figure 3. Profile of P Diffusion in the B5 Sediment from the TNX OD after 3 Months of

Contact with Biological P (BP) and North Carolina (NCA): Laboratory Study 24

Figure 4. Profile of Fe Diffusion in the B5 Sediment from the TNX OD after 3 Months of Contact with $\mathrm{Fe}(0)$ : Laboratory Study.... 25

Figure 5. Transport of P and Fe in the Horizontal Treatments; the Total Evaluated Depth from the Element Source was $5 \mathrm{~cm}$. 27

Figure 6. Transport of $\mathrm{P}$ and Fe in the Vertical Treatments; the Evaluated Distance for Element was $3 \mathrm{~cm}$ 28

\section{LIST OF TABLES}

Table 1. Commercially Available Phosphate Materials Tested for Impurities ........................ 7

Table 2. Particle Size Distribution of Phosphate Materials ................................................ 12

Table 3. Total Concentration* ( $\mathrm{mg} / \mathrm{kg}$ ) of Impurities in Phosphate Materials and TNX OD

Background Sediment ........................................................................................ 14

Table 4. Concentration (mg/kg) of Impurities in Tested Phosphate Sources ....................... 15

Table 5. Total Concentration* (mg/kg) of Impurities in Tested Phosphate Origin ............... 16

Table 6. Concentration of Elements* (mg/kg) in TCLP Extract of Phosphate Sources......... 17

Table 7. Concentration of Elements* (mg/kg) in TCLP Extract of Phosphate Origin........... 18

Table 8. Solubility of Phosphate Materials in Comparison with Some Phosphate Mineral Phases; the Solubility was Calculated by Following Davies Equation (Sposito, 1986) .. 19

Table 9. Concentration of Elements* (mg/kg) in Water Soluble Fraction of Phosphate

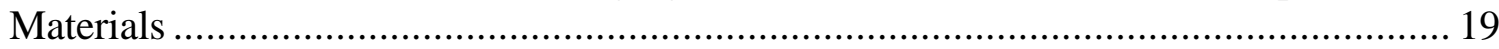

Table 10. Effective Diffusion Coefficients $\left(\mathrm{cm}^{2} / \mathrm{s}\right)$ Calculated from Field Data, Laboratory Data, and $K_{d}$ Values

Table 11. Diffusion of P or Fe in Treatments with Phosphate Source or Fe(0); Laboratory Experiment, Average Values Based on Two Replicates ............................................... 23

Table 12. Calculated P and Fe Effective Diffusion Coefficients (Deff) Based on Laboratory and Field study. Calculated Radius of Sediment Amendment Zone-of-Influence Based on Field Study 
WSRC-TR-2003-00579, REV. 0

\section{LIST OF ACRONYMS}

Bdl

BP

CEC

$D_{\text {eff }}$

ICP-AES

$\mathrm{K}_{\mathrm{d}}$

na

NCA

Proc.

RCRA

RP

SRS

TNX OD

ZOI below detection limit

Biological Phosphate

Cation Exchange Capacity

Effective Diffusion Coefficient

Inductively Coupled Plasma - Atomic Emission Spectroscopy

Distribution Coefficient

not analyzed

North Carolina Apatite

processed

Resource Conservation and Recovery Act

rock phosphate

Savannah River Site

TNX Outfall Delta

Zone-of-Influence 
WSRC-TR-2003-00579, REV. 0

\section{EXECUTIVE SUMMARY}

The TNX pilot-scale research facility released processed waste, containing elevated concentrations of several metals and radionuclides into an unlined seepage basin between 1958 and 1980. The contents of this basin have entered the nearby swamp, the TNX Outfall Delta (TNX OD), by subsurface and overland flow. Studies were conducted to evaluate whether sediment amendments could be used to reduce contaminant mobility and bioavailability. Previous studies showed that the addition of a phosphate mineral, apatite, and zero-valent iron, $\mathrm{Fe}(0)$, were effective at immobilizing a broad range of contaminants at the site (Kaplan et al., 2002). It is anticipated that the sediment amendments will be broadcast on the ground surface and backfilled into drilled $2 \mathrm{~cm}$ diameter x $15 \mathrm{~cm}$ deep holes spaced across the contaminated area. The amendments' zone-of-influence of these two application methods was conducted to permit treatment design. The objective of this study was to determine 1) which source of phosphate mineral is most suitable for sedimentcontaminant stabilization, and 2) what is the extent of the zone-of-influence of applied apatite and $\mathrm{Fe}(0)$.

Nine commercially available phosphate minerals, representing $>95 \%$ of the phosphate production in the country, were evaluated for solubility, concentration of trace metals, and leachability of trace metals. Processed and mined rock phosphate contain high total concentrations of arsenic (As), cobalt (Co), chromium (Cr), copper $(\mathrm{Cu})$, and strontium $(\mathrm{Sr})$; however, they did not exceed the Resource Conservation and Recovery Act (RCRA) toxicity characteristic leaching procedure (TCLP) limits. The use of stronger extractants indicated that these elements were very strongly bound by most apatite material; therefore, if they were applied to contaminated sediment at a rate sufficient for remediation, they would not pose an environmental risk. The biogenic apatite (fish bone) contained significantly lower metal impurities than processed and mined rock phosphate and was appreciably more soluble, i.e., it had a $\log \mathrm{K}_{\text {sp }}$ of -45.2 compared to -57.0 for the mined rock phosphate samples.

There are many phosphate sources that can safely and effectively be applied to the TNX OD. By combining biogenic and mined phosphate it will be possible to obtain a wide range of phosphate solubility, permitting rapid immobilization of contaminants, while at the same time providing a slow release of phosphate for continued sediment treatment. Addition of $\mathrm{Fe}(0)$ is known to be very effective at removing $\mathrm{As}, \mathrm{Cr}$, Co and other elements from the aqueous phase. Therefore, its application at the TNX OD could significantly immobilize these contaminants.

The ZOI of surface applied amendments (horizontally applied), and amendments backfilled into holes (vertically applied) were evaluated under field conditions. Additionally, vertical application of ZOI was tested in the lab. For the surface applied amendments in the13-month field study, P from the biological apatite and from the mined apatite moved $\sim 3 \mathrm{~cm}$; and $\mathrm{Fe}$ from the $\mathrm{Fe}(0)$ treatment moved $\sim 5 \mathrm{~cm}$. Retardation factors were calculated and used to estimate the zone-of-influence at various intervals. To provide a measure of lateral movement of the $\mathrm{P}$ and $\mathrm{Fe}$ in the vertical treatments, field and laboratory data were collected and applied to diffusion equations (Fick's second law) to provide effective diffusion coefficients. Both sets of data were reasonably consistent, indicating that $\mathrm{P}$ from the 
biological and mined apatite and $\mathrm{Fe}$ from $\mathrm{Fe}(0)$ would be expected to diffuse more than $5 \mathrm{~cm}$ after 10 years.

It will not be possible to apply $\mathrm{Fe}(0)$ with apatite to the contaminated sediment at the TNX OD because an iron-phosphate phase precipitates, greatly reducing the mobility, and therefore the zone-of-influence of iron and phosphate. If both amendments are deemed necessary, it would be best to apply them at different times.

\section{INTRODUCTION}

\subsection{BACKGROUND}

The TNX pilot-scale research facility released process waste into an unlined seepage basin between 1958 and 1980. The basin, referred to as the Old TNX Seepage Basin, was designed to contain wastewater until it could seep into the underlying sediments. It was anticipated that the sediment would then impede contaminant migration. The waste discharged to the Old TNX Seepage Basin included chromium, mercury, sodium, uranium and thorium. The basin contents have entered the nearby inner swamp by subsurface and overland flow; the overland flow is the result of draining operations and overflow of the basin during closure in 1981.

\subsection{IMMOBILIZATION OF CONTAMINANTS BY PHOSPHATE AND FE(0)}

Researchers have reported that apatite, a calcium-phosphate mineral, immobilizes lead $(\mathrm{Pb})$ and other ions such as manganese $(\mathrm{Mn})$, cobalt $(\mathrm{Co})$, copper $(\mathrm{Cu})$, cadmium $(\mathrm{Cd})$, zinc $(\mathrm{Zn})$, magnesium $(\mathrm{Mg})$, barium $(\mathrm{Ba})$, uranium $(\mathrm{U})$, or thorium $(\mathrm{Th})$ in contaminated media (Ma et al., 1995; Ryan et al., 2001; Seaman et al., 2001; Suzuki, et al., 1981; Suzuki, et al., 1982). Immobilization of these elements occurs due to precipitation, adsorption and isomorphic substitution. Wright et al. (1995) reported that the immobilization of Pb by apatite was primarily through a process of apatite dissolution followed by precipitation of various pyromorphite-type minerals under acidic conditions, or the precipitation of hydrocerussite $\left[\mathrm{Pb}_{3}\left(\mathrm{CO}_{3}\right)_{2}(\mathrm{OH})_{2}\right.$ or $\left.\mathrm{Pb}(\mathrm{OH})_{2}\right)$ and lead oxide fluoride $\left(\mathrm{Pb}_{2} \mathrm{OF}_{2}\right)$ under alkaline conditions. Otavite $\left(\mathrm{CdCO}_{3}\right)$, cadmium hydroxide $\left[\mathrm{Cd}(\mathrm{OH})_{2}\right]$, and zincite $(\mathrm{ZnO})$ were formed in the $\mathrm{Cd}$ or Zn systems, respectively, especially under alkaline conditions. Phosphate-metal precipitates are typically very stable.

Suzuki et al. (1981 and 1982) concluded that the removal of $\mathrm{Pb}, \mathrm{Cu}, \mathrm{Mn}, \mathrm{Co}, \mathrm{Cd}, \mathrm{Zn}, \mathrm{Ni}$, and Ba from contaminated medium was due not only to adsorption but also to an ion-exchange reaction between the cations in solution and the $\mathrm{Ca}^{2+}$ ions of the apatite. Suzuki et al. (1982) reported that the order of the ions according to the amount exchanged was as follows: $\mathrm{Pb}^{2+}>\mathrm{Cu}^{2+}>\mathrm{Mn}^{2+}=\mathrm{Co}^{2+}$. In another paper, Suzuki et al. (1981) reported that the ranking of the ions according to amount exchanged was as follows: $\mathrm{Cd}^{2+}, \mathrm{Zn}^{2+}>\mathrm{Ni}^{2+}>\mathrm{Ba}^{2+}, \mathrm{Mg}^{2+}$. Data presented by Knox et al., (2003) are consistent with those of Suzuki et al. (1981, 1982), in that the apatite was more effective at reducing aqueous $\mathrm{Pb}$ than aqueous $\mathrm{Co}$ or $\mathrm{Ba}$ concentrations. 
Isomorphic substitution is a process in which the contaminant substitutes for $\mathrm{Ca}$, phosphate, or hydroxide in the apatite structure. This removal mechanism is highly desirable from the standpoint of contaminant stabilization because the contaminant becomes incorporated into the structure of the apatite. Isomorphic substitution is common in apatite because its crystalline structure is very flexible. Several different elements can substitute into its structure. Calcium exists in apatite in 7-fold and 9-fold coordination. The 7-fold coordinated $\mathrm{Ca}$ ions are about $10 \%$ smaller than the 9 -fold coordinated ions. This difference in ionic radius permits a large range of contaminants to substitute for $\mathrm{Ca}$, including the following constituents: $\mathrm{Sr}, \mathrm{Ra}, \mathrm{Pb}, \mathrm{Cs}, \mathrm{Th}, \mathrm{U}(\mathrm{VI})$, and $\mathrm{Cr}(\mathrm{VI})$ (Deer et al., 1975). Elements that can substitute for phosphate $(0.254 \mathrm{~nm}$ radius $)$ include $\mathrm{TcO}_{4}{ }^{-}(0.268 \mathrm{~nm})$ and $\mathrm{CO}_{3}{ }^{2-}(0.211 \mathrm{~nm})$. Finally, the hydroxyl anion $(0.153 \mathrm{~nm})$ can be substituted with $\mathrm{Br}^{-}(0.196 \mathrm{~nm}), \mathrm{Cl}^{-}(0.181$ $\mathrm{nm})$ and $\mathrm{F}^{-}(0.136 \mathrm{~nm})$.

The other sediment amendment evaluated in this study was $\mathrm{Fe}(0)$. $\mathrm{Fe}(0)$ removes metals from the aqueous phase by three primary processes: reductive precipitation (by $\mathrm{Fe}(0)$ or $\mathrm{Fe}(\mathrm{II})$ ), coprecipitation with $\mathrm{Fe}(\mathrm{II} / \mathrm{III})$, and metal sorption by $\mathrm{Fe}(0)$-originating, $\mathrm{Fe}(\mathrm{II} / \mathrm{III})$ oxyhydroxides. Reductive precipitation involves the transfer of electrons from $\mathrm{Fe}(0)$ to a constituant that is less soluble in the reduced than in the oxidized form. Common contaminants that can be removed from the aqueous phase in this manner are $\mathrm{Cr}, \mathrm{Hg}, \mathrm{Mo}, \mathrm{Tc}$, and $\mathrm{U}$. For example, the reductive precipitation of $\mathrm{Cr}(\mathrm{VI})$ can be described by Equation 1:

$$
\mathrm{CrO}_{4}{ }^{2-}+1.5 \mathrm{Fe}^{0}+5 \mathrm{H}^{+}=\mathrm{Cr}(\mathrm{OH})_{3}+3 \mathrm{H}_{2} \mathrm{O}+1.5 \mathrm{Fe}^{2+} .
$$

In this example, $\mathrm{CrO}_{4}{ }^{2-}$ is both more mobile and toxic than $\mathrm{Cr}(\mathrm{OH})_{3}$, which is predominantly in the solid phase.

As Equation 1 shows, $\mathrm{Fe}^{2+}$ ions are released and acidity is consumed during the reaction. Both of these changes are conducive to the formation of Fe(II/III)-oxyhydroxides. If other metals are present, they may coprecipitate with the Fe(II/III)-oxyhydroxide. Chrome removal by $\mathrm{Fe}(0)$ is believed to occur primarily through this reaction, whereby $\mathrm{Cr}, \mathrm{Fe}(\mathrm{OH})_{3}$ solid solutions are formed (Eary and Rai, 1987; Sass and Rai, 1987).

$\mathrm{Fe}(0)$ can remove aqueous contaminants by first oxidizing to form Fe(II/III)-oxyhydroxides, which then can act as a sorbent. The Fe(II/III)-oxyhydroxides increase the sorption capacity of the system. Contaminant removal in this manner is the least desirable of the three removal mechanisms due to the weak nature of the bond between the contaminant and the $\mathrm{Fe}(\mathrm{II} / \mathrm{III})$ oxyhydroxide.

\subsection{PHOSPHATE ROCK - PHOSPHOROUS SOURCES}

Phosphate rock minerals are the only significant global resources of phosphorus. The United States (U.S.) is the world's leading producer and consumer of phosphate rock. The southern states (Florida and North Carolina) and western phosphate fields (mostly Idaho and Utah) are the major mining areas in the U.S. Phosphate rock is produced by 11 companies at 18 mines; 12 mines in Florida and one in North Carolina accounts for $86 \%$ of domestic production. 
WSRC-TR-2003-00579, REV. 0

Southern phosphate-rich deposits are of marine origin from Miocene and Pliocene ages, i.e., approximately 5-10 million years old. Biological and chemical changes transformed the phosphate that existed in the sea into the phosphate sediment that we mine today. There are many theories about how the Florida phosphate deposit was formed. One of the most common theories is that during the Miocene era, deep seawater currents flowed up onto topographically high areas. These upwelling currents caused nutrient and phosphate rich water to rise to the surface of the sea that covered Florida at the time. The phosphate precipitated from the seawater to form phosphate-rich sediment that solidified into nodules (Kohn et al., 2002). As time passed, sea levels dropped and phosphate and limestone were exposed as land. During the Pleistocene era, the marine phosphate deposits were geologically reworked and re-deposited in a concentrated form. Florida's phosphate deposit contains the teeth, bones and waste excrement from marine life and also the bones of terrestrial pre-historic animals such as mastodons, saber-tooth tigers, bears, camels, threetoed horses and other ancient animals.

The western phosphate deposits, Permian Phosphoria Formation, are older deposits that formed off the western shores of the Pangean super continent in what is today southeastern Idaho and surrounding states (Montana, Wyoming, Colorado, Utah, and Nevada). The Phosphoria Formation is considered a "super giant" among phosphate deposits, covering about $340,000 \mathrm{~km}^{2}$ and containing five to six times more phosphorous than the total phosphorous budget in today's oceans (Knudsen and Gunter, 2002).

\subsection{OBJECTIVE}

The main objectives of this study were:

1) to determine which natural source of phosphate would be most suitable for remediating contaminated sediments (nine phosphate materials were tested),

2) to determine the Zone-of-Influence (ZOI) of two phosphate materials and $\mathrm{Fe}(0)$ in the TNX OD sediment under laboratory conditions, and

3 ) to determine the $\mathrm{ZOI}$ of two phosphate materials, $\mathrm{Fe}(0)$, and mixture of each phosphate material with $\mathrm{Fe}(0)$, applied horizontally or vertically, in the field (TNX OD).

Three types of phosphate sources were initially evaluated: mined rock phosphate (Florida, Idaho, North Carolina, and Tennessee), processed rock phosphate (North Pacific AG Products; Montana Natural Granulated Rock Phosphate, Spring Phosphate Powdered, and Volcanaphos) and a biogenic apatite (fish bones). Collected samples were analyzed for solubility, toxicity characteristic leaching procedure (TCLP), extractable metals, total elemental content and selective extraction metal content.

In the ZOI laboratory and field studies, two types of phosphate materials were tested: mined apatite from North Carolina and biological apatite (ground bones, reviewed at www.pimsnw.com), and zero-valent iron, $\mathrm{Fe}(0)$. Apatite is a common subsurface and surface sediment amendment for metal immobilization, whereas $\mathrm{Fe}(0)$ is not as commonly used for surface sediment applications as it is used for subsurface permeable reactive barriers. 
WSRC-TR-2003-00579, REV. 0

\section{MATERIALS AND METHODS}

A detailed description of the laboratory and field procedure used in this study is presented in Appendix B: Work Instruction for the Laboratory Study and Appendix C: Work Instruction and Additional Results for the Field Study. The following is a brief synopsis.

\subsection{LABORATORY STUDIES}

\subsubsection{Characteristics of Phosphate Sources}

Nine phosphate materials (Table 1) were tested to determine which natural source of phosphate would be most suitable for remediation of contaminated sediments. The tested materials were collected from three sources of phosphate, mined rock phosphate (Florida, Idaho, North Carolina, and Tennessee), processed rock phosphate (North Pacific AG Products; Montana Natural Granulated Rock Phosphate, Spring Phosphate Powdered and Volcanaphos) and a biogenic apatite (fish bones) (Table 1). The collected samples were analyzed for $\mathrm{pH}$, particle size distribution, organic matter content, solubility, toxicity characteristic leaching procedure (TCLP) extractable metals, and total elemental content. The phosphate material $\mathrm{pH}$ was determined from a 1:1 mineral/water equilibration solution (Thomas, 1996). Percent organic carbon was estimated by loss-on-ignition at a temperature of $375^{\circ} \mathrm{C}$. Particle size distribution (percent sand, silt, and clay) was determined by the micro-pipette method (Miller and Miller, 1987). The solubility test was run in $50 \mathrm{~mL}$ centrifuge tubes with $1 \mathrm{~g}$ of well-mixed apatite sample and $10 \mathrm{~mL}$ of DI water. The samples were on the shaker for 12 days. The samples were then centrifuged and the solution was decanted. The decanted solution was analyzed for $\mathrm{pH}$ and then acidified and analyzed for $\mathrm{Al}$, As, $\mathrm{Ba}, \mathrm{Cd}, \mathrm{Ce}, \mathrm{Co}, \mathrm{Cr}, \mathrm{Cu}, \mathrm{Fe}, \mathrm{K}, \mathrm{Mg}, \mathrm{Mn}, \mathrm{Mo}, \mathrm{Na}, \mathrm{Ni}, \mathrm{Pb}, \mathrm{P}$, and $\mathrm{Sr}$ by ICP-AES. A detailed description of the laboratory procedure used in this study is presented in Appendix B: Work Instruction for the Laboratory Study. All phosphate materials were extracted with the USEPA standardized Toxicity Characteristic Leaching Procedure (TCLP; USEPA, 1992). The TCLP leaching solution is comprised of $0.1 M$ glacial acetic acid and $0.0643 M \mathrm{NaOH}$, with a final $\mathrm{pH}$ of 4.93 . Forty milliliters of leaching solution were added to $2 \mathrm{~g}$ of phosphate material; the mixture was agitated on a shaker for 18 hours at $25^{\circ} \mathrm{C}$, and then centrifuged. After centrifugation, the supernatants were filtered through $0.22 \mu \mathrm{m}$ pore-size polycarbonate filters, acidified to $1 \% \mathrm{HNO}_{3}$, and analyzed for metals by ICP-AES.

\subsubsection{Laboratory Measurements of Zone-of-Influence}

To determine the zone-of-influence (ZOI) of sequestering materials the following experiments were conducted: phosphate sorption isotherm, ferrous iron sorption isotherm and laboratory experiment of zone-of-influence for vertical application of $\mathrm{Fe}(0)$ and two types of apatite.

\subsubsection{Sorption Isotherm Experiments}


The objective of the phosphate and ferrous iron sorption isotherm experiments was to measure the phosphate or iron distribution coefficient, $\mathrm{K}_{\mathrm{d}}$, in the TNX OD sediment (from coordinate B5) to permit calculation of the effective diffusion coefficient, $\mathrm{D}_{\text {eff, }}$ using Fick's second law (equations 1 and 2) and equation 3 (Dragun, 1998):

$$
\begin{aligned}
& \qquad \delta \mathrm{c} / \delta \mathrm{t}=\left(\mathrm{D}_{\mathrm{eff}} / \mathrm{R}_{\mathrm{F}}\right)\left(\delta^{2} \mathrm{c} / \delta \mathrm{x}^{2}\right) \\
& \mathrm{c} \quad=\text { concentration of a solute }\left(\mathrm{mg} / \mathrm{cm}^{3}\right) \\
& \mathrm{t}=\text { time }(\mathrm{s}) \\
& \mathrm{D}_{\mathrm{eff}}=\text { diffusion coefficient }\left(\mathrm{cm}^{2} / \mathrm{s}\right) \\
& \mathrm{R}_{\mathrm{F}}=\text { retardation factor }(\text { calculated from Equation } 2) \\
& \mathrm{x} \quad=\text { direction of diffusion }(\mathrm{cm}) \\
& \qquad \mathrm{R}_{\mathrm{F}}=1+\left(\rho_{\mathrm{b}} / \phi_{\mathrm{e}}\right) \mathrm{K}_{\mathrm{d}}
\end{aligned}
$$

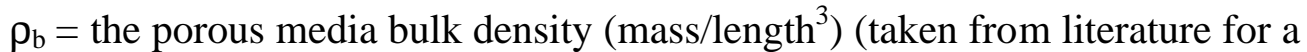
sediment with the texture of sediment B5, loamy sand, $1.55 \mathrm{~g} / \mathrm{cm}^{3}$ ) (Dragun, 1998)

$\phi_{\mathrm{e}}=$ the effective porosity at saturation of media (taken from literature for a sediment with the texture of sediment B5, loamy sand, 0.47) (Hillel, 1980)

$$
\mathrm{D}_{\mathrm{eff}}=\mathrm{D}_{\mathrm{o}} / \mathrm{R}_{\mathrm{F}}
$$

$\mathrm{D}_{\text {eff }}=$ diffusion coefficient,

$\mathrm{D}_{\mathrm{o}}=$ the aqueous diffusion coefficient (taken from literature),

$\mathrm{R}_{\mathrm{F}}=$ retardation factor (calculated from equation 2)

The experiment was conducted in $50 \mathrm{ml}$ centrifuge tubes, where $2 \mathrm{~g}$ of well-mixed B5 sediment from the TNX OD was pre-equilibrated with non-contaminated TNX OD swamp water. Then the sediment was shaken for 4 days with a solution made of the TNX OD swamp water and an appropriate volume of $\mathrm{PO}_{4}{ }^{3-}$ (from $\mathrm{NaH}_{2} \mathrm{PO}_{4}-\mathrm{H}_{2} \mathrm{O}$ ) or $\mathrm{Fe}^{2+}$ (from $\mathrm{FeCl}_{2}$ ) solution (Tables $1 \mathrm{~B}$ and $2 \mathrm{~B}$, respectively). The samples were then centrifuged and the $\mathrm{pH}, \mathrm{P}$ (modified ascorbic acid method; Kuo, 1996) and $\mathrm{Fe}^{2+} / \mathrm{Fe}^{3+}(1,10$ - phenanthroline method; Sparks, 1996) were measured. 
Table 1. Commercially Available Phosphate Materials Tested for Impurities

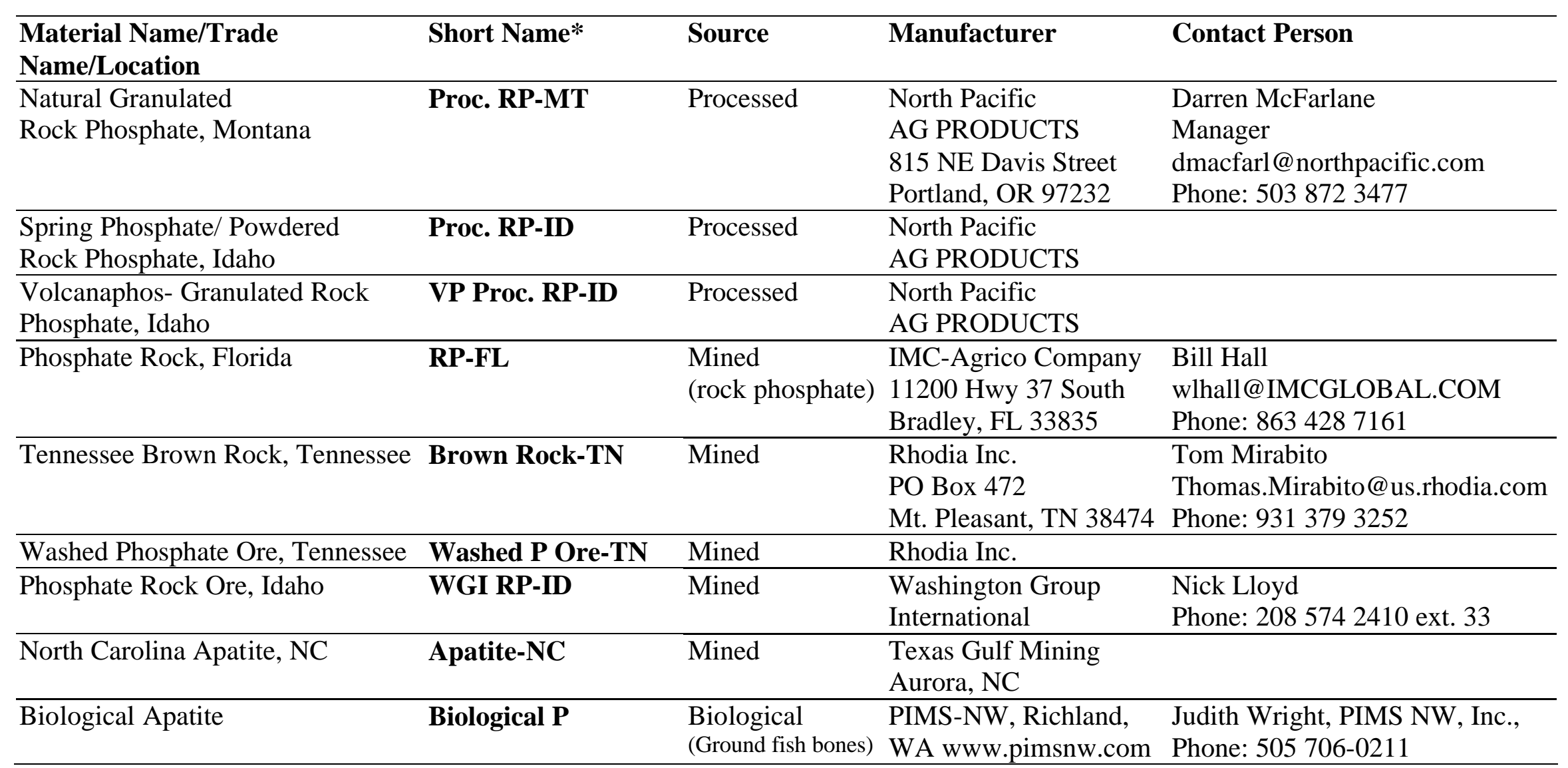

* The short name will be used throughout document 
WSRC-TR-2003-00579, REV. 0

\subsubsection{Zone-of-Influence for Vertical Application of $\mathrm{Fe}(0)$ and Apatite}

The objective of this study was to determine horizontal (lateral) movement of $\mathrm{P}$ and $\mathrm{Fe}$ in sediment with vertical application of amendments. Two types of phosphate materials, mined apatite from North Carolina and biological apatite (ground fish bones, reviewed at www.pimsnw.com), and zero-valent iron, $\mathrm{Fe}(0)$ - granular Fe(0) (Peerless Supply, Columbus, $\mathrm{OH})$ were tested. The sediment used in this study came from coordinate B5 in the TNX OD study site. This sediment was used because it had relatively high concentrations of constituents of interest, yet, based on analytical results, was not classified as either hazardous or radioactive. The sediment sample was collected from the top $15 \mathrm{~cm}$, but did not include the surface organic mat, i.e., the $\mathrm{O}$ sediment horizon.

Four $\mathrm{cm}$ of saturated sediment (B5, TNX OD) and $1.1 \mathrm{~cm}$ of amendment (apatite of $\mathrm{Fe}(0)$ ) was placed in $60 \mathrm{~mL}$ syringes. The syringes with the saturated sediment were covered with aluminum foil to exclude light, sealed, and placed vertically on the lab bench for a period of 3 months. On August 12, 2003, the bottoms of the syringes were cut off and the samples were gently extrude out and sliced into 0.3 or $0.5 \mathrm{~cm}$ thick slices. Each slice was collected as an individual sample. Each collected sample was digested with $\mathrm{HNO}_{3} / \mathrm{H}_{2} \mathrm{SO}_{4} / \mathrm{HCl}$, and the digested extracts were analyzed for total content of P, Fe and other elements by ICP-AES.

\subsection{THE FIELD STUDY}

\subsubsection{Zone-of-Influence for Vertical and Horizontal application $\mathrm{Fe}(0)$ and Apatite}

\subsubsection{Experimental Design and Sample Collection}

The objective of the field study was to determine the zone-of-influence for amendments under field conditions in the TNX OD wetland sediments. There were two application methods (horizontal and vertical), six amendment (Control - no amendment, NC Apatite, Bio-apatite, $\mathrm{Fe}(0)$, $\mathrm{NC}$ Apatite $+\mathrm{Fe}(0)$, and Bio-apatite $+\mathrm{Fe}(0))$ and three replicates for a total of thirty six plots (Table 1C and Figure 1). Vertical treatments were performed by augering $2 \mathrm{~cm}$ diameter $\mathrm{x} 15 \mathrm{~cm}$ deep holes into the ground and that backfilled with the amendment. Horizontal treatments were applied by spreading amendments over the surface of the sediment in a $30 \mathrm{~cm}$ by $30 \mathrm{~cm}$ plot. Approximately $163 \mathrm{~g}$ of each amendment was applied to the $30 \mathrm{~cm}$ by $30 \mathrm{~cm}$ plot; at a rate equal to $18,111 \mathrm{~kg} / \mathrm{ha}$.

Sediment samples from the horizontal and vertical treatments were collected before and 13 months after amendment application. Prior to the application of amendments, samples from the horizontal and vertical treatment areas were collected with a sediment auger into plastic sleeves $(2 \mathrm{~cm}$ diameter $\mathrm{x} 13$ to $25 \mathrm{~cm})$. The sediment samples were collected from the center of each plot. Table 5C shows the scheme in which the samples were collected after 13 months of amendment application, labeled, and split. The sediment samples from the horizontal treatment areas were collected with a sediment sampler into plastic sleeves. Sediment samples were collected from the center of each plot (next to the hole after the first sediment sampling). Collected sediment cores were sliced into $1 \mathrm{~cm}$ increments and each 
subsample was analyzed. The sediment samples from vertical treatments were collected from the center of each plot (next to the hole after the first sediment sampling) using a sediment profiler (18cm deep x $10 \mathrm{~cm}$ wide and $2 \mathrm{~cm}$ thick). The collected sediment profile was divided vertically into $1 \mathrm{~cm}$-wide strips.

\subsubsection{Methods}

A homogenized sample of all collected sediment samples from vertical, horizontal and control treatments before amendment application was sent to Savannah River Technology Center, Analytical Development Section for TCLP extraction.

The second set of sediment samples, collected almost 13 months after amendment application, was analyzed for total concentration of elements with a focus on $\mathrm{P}$ or $\mathrm{Fe}$ in extracts from a total digestion with $\mathrm{HNO}_{3} / \mathrm{H}_{2} \mathrm{SO}_{4} / \mathrm{HCl}$, performed on microwave system STAR 6. From the horizontal plots, each slice from two replicates of each treatment was analyzed. From the vertical plots, each slice of one replicate of each treatment was analyzed. For more details please see Appendix C, Table 6C. All extracts from the total digestion were analyzed by ICP-AES. 
WSRC-TR-2003-00579, REV. 0

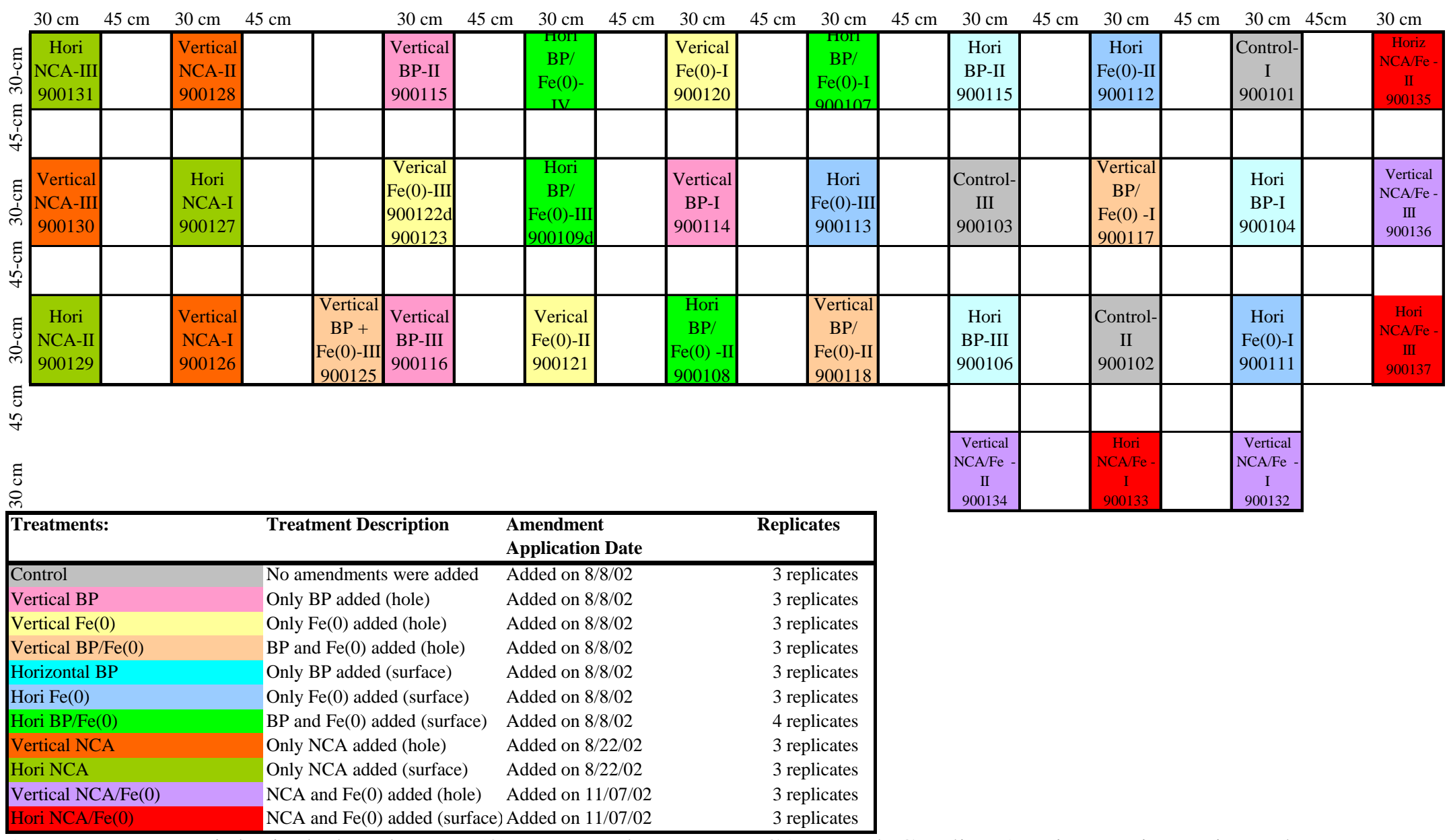

Acronyms: BP - Biological Phosphate, Fe(0) - Zero Valent Iron , NCA - North Carolina Apatite, Hori - Horizontal I, II, III, IV - replicates

Figure 1. Experimental Design of the Field Study of Zone-of-Influence (ZOI) at the TNX OD 
WSRC-TR-2003-00579, REV. 0

\subsection{SAMPLE ANALYSIS AND QUALITY ASSURANCE}

All work conducted in these studies followed Standard QA practices described in the WSRC Manual 1Q. The $\mathrm{pH}$, total concentration of $\mathrm{P}$ and $\mathrm{Fe}$ and all sediment characterization measurements were made by SRTC personnel following standard procedures described in detail by Sparks (1996). Blanks and spikes were included whenever it made technical sense to do so (e.g., there is no blank control for $\mathrm{pH}$ measurement). Duplicates were conducted approximately every 15 analyses. The ICP-AES analyses were conducted by the Environmental Protection Department, Environmental Monitoring Section Laboratory, Westinghouse Savannah River Company. This SC DHEC-certified lab included and provided the results of laboratory blank and spike controls. Data that were outside of the certificate guidelines were not reported in this report. There were limited problems with the Th and $\mathrm{U}$ data; none of these compromised data are included in the report.

The lab notebook used for this study is WSRC-NB-2002-00142.

\section{RESULTS AND DISCCUSION}

\subsection{PHOSPHATE MATERIAL CHARACTERIZATION}

The nine phosphate materials (see Table 1) were evaluated for remediation of contaminated sediments. The tested materials were collected from three sources of phosphate: mined rock phosphate (Florida, Idaho, North Carolina, and Tennessee), processed rock phosphate (North Pacific AG products, Montana Natural Granulated Rock Phosphate, Spring Phosphate Powdered and Volcanaphos) and a biogenic apatite (ground fish bones - www.pimsnw.com; PIMS-NW, Richland, WA). These phosphate materials had a pH range of 5.84 to 7.37, with the lowest $\mathrm{pH}$ values for processed phosphate materials $(\mathrm{pH} 5.84)$ and the highest for mined phosphate (pH 7.37) (Table 2). Loss-on-ignition is an approximation of organic carbon content. Organic carbon content was very high for the biological, $34.2 \%$ and only $4.3 \%$ for mined phosphate materials (Table 2). Particle size analysis showed that the texture of the phosphate materials varied substantially (Table 2$)$. The clay fraction $(<2 \mu \mathrm{m})$ was the least abundant and ranged from $0.2 \%$ (Biological P) to $1.2 \%$ (Brown Rock-TN). The silt fraction, $2-50 \mu \mathrm{m}$, was substantially more common than the clay fraction. The highest silt content was obtained from processed phosphate materials with a mean of $42.8 \%$. The sand fraction, $50 \mu \mathrm{m}-1000 \mu \mathrm{m}$, was the most abundant with means of $52.4 \%, 60.1 \%$, and $75.1 \%$ for processed, mined, and biological phosphate, respectively. The biological and mined phosphate materials had the highest gravel fraction (Table 2). 
WSRC-TR-2003-00579, REV. 0

Table 2. Particle Size Distribution of Phosphate Materials

\begin{tabular}{|c|c|c|c|c|c|c|c|}
\hline Phosphate & Source & $\mathrm{pH}$ & $\begin{array}{l}\text { Organic } \\
\text { Carbon }\end{array}$ & Gravel & Sand & Silt & Clay \\
\hline Materials $^{(a)}$ & & & $(\%, w t)$ & $(\%, w t)$ & $(\%, w t)$ & $(\%, \mathrm{wt})$ & $(\%, \mathrm{wt})$ \\
\hline Proc. RP-MT & Processed & & & 0.0 & 60.8 & 38.6 & 0.7 \\
\hline Proc. RP-ID & & & & 10.9 & 36.6 & 51.6 & 0.9 \\
\hline VC Proc.-ID & & & & 0.8 & 59.9 & 38.2 & 1.1 \\
\hline Avg & & 5.84 & na & 3.9 & 52.4 & 42.8 & 0.9 \\
\hline (stdev) & & 5.68 & & 6.1 & 13.7 & 7.7 & 0.2 \\
\hline PR-FL & Mined & & & 0.0 & 39.0 & 60.4 & 0.7 \\
\hline Brown Rock-TN & & & & 8.3 & 65.4 & 25.2 & 1.2 \\
\hline Washed P Ore-TN & & & & 4.1 & 69.4 & 25.5 & 1.1 \\
\hline WGI-RP-ID & & & & 39.9 & 29.4 & 30.0 & 0.7 \\
\hline Apatite-NC & & & & 0.0 & 97.6 & 2.4 & 0.0 \\
\hline Avg & & 7.37 & 4.3 & 10.5 & 60.1 & 28.7 & 0.7 \\
\hline (stdev) & & 7.74 & & 16.8 & 27.0 & 20.7 & 0.5 \\
\hline Biological P & Biological $^{(b)}$ & 7.10 & 34.2 & 15.5 & 75.1 & 9.1 & 0.2 \\
\hline
\end{tabular}

(a) Abbreviations for minerals are defined in Table 1.

(b) Only one sample analyzed.

na - not analyzed.

High concentrations of some elements in the tested phosphate materials were expected because it is well known that these elements become concentrated into the structure of the apatite minerals. Metal concentration in apatite is the very mechanism on which this technology (in-situ immobilization) attempts to capitalize. Total concentrations of impurities in nine tested phosphate materials in comparison to the background values for surface sediment of the TNX OD (Kaplan et al., 2002) are presented in Table 3. The concentrations of several tested elements e.g., $\mathrm{Cr}, \mathrm{Cu}$, and $\mathrm{Sr}$ were higher in all phosphate sources relative to the background values (Table 3). The Sr concentrations reported in Table 3 are from the stable isotope, not the radioactive isotope; thus, its high concentration, e.g., $815 \mathrm{mg} / \mathrm{kg}$ in the Biological-P, is not, in itself, of great concern. Also, As concentration was high for almost all tested materials; only As content in Biological P $(<0.7)$ was lower than the background value $(1.5 \mathrm{mg} / \mathrm{kg})$.

Arsenic, $\mathrm{Co}$, and $\mathrm{Cu}$ concentrations were among the most common impurities in the processed phosphate material (Table 4). The data presented in Table 4 show very high standard deviations, likely resulting from the varying geological origins of these materials. Therefore, the data in Table 5 are presented by the origin of phosphate. The standard deviations associated with these means do not vary nearly as much as the deviations in Table 4. The phosphate materials from Montana and Idaho generally had higher total concentrations of all tested elements because these deposits belong to the western phosphate deposition (Permian Phosphoria Formation), which is an older deposition than the Florida phosphate deposits and, therefore, they have a higher concentration of impurities. The older 
phosphate deposits have a longer contact time with surrounding rock and thus have had more time to scavenge elements into their structure (Wright, 1995).

Biological P had the lowest total concentrations for almost all elements. Low concentrations in biological phosphate could be due to a short accumulation time (Wright 1995). After deposition in sediments, biogenic apatite incorporates trace elements at concentration levels that are enriched by one to many orders of magnitude over the concentration levels in the surrounding aqueous solutions (Wright 1990). This comparison of biogenic apatite in vivo to biogenic apatite exposed to natural water illustrates that apatite can act to greatly concentrate trace elements.

The toxicity characteristic leaching procedure (TCLP) is a regulatory test widely used to classify materials as hazardous or nonhazardous (U.S. EPA, 1990). Comparing the results of the extract analysis of phosphate materials to the regulatory levels indicated that only $\mathrm{Cd}$ concentrations in the processed phosphate materials exceeded $(1.3 \mathrm{mg} / \mathrm{kg})$ regulated TCLP limits $(1.0 \mathrm{mg} / \mathrm{kg})($ Table 6$)$. However, the difference was not statistically significant. In Table 7, the results are presented by the phosphate origin. The materials from older phosphate deposits e.g., Montana, Idaho, and Tennessee generally had higher concentrations of all tested elements in the TCLP extract; however, these concentrations were still lower than regulated TCLP limits (Table 7).

The solubility test showed that Biological $\mathrm{P}$ was appreciably more soluble than mined rock phosphate, i.e., Biological P had a $\log (\mathrm{Ksp})$ of -45.2 compared to -57.0 for the mined rock phosphate (Table 8). Also the biological apatite had the highest $\mathrm{P}$ concentrations in the water soluble fraction, $505 \mathrm{mg} / \mathrm{kg}$ (Table 9). The total concentration of $\mathrm{Sr}$ in biological apatite was one of the highest; however, Sr concentration in the water soluble fraction was low, even lower than for processed phosphate materials, i.e., $2.56 \mathrm{mg} / \mathrm{kg}$ and $4.52 \mathrm{mg} / \mathrm{kg}$, respectively, for Biological $\mathrm{P}$ and processed phosphate materials. 
Table 3. Total Concentration* (mg/kg) of Impurities in Phosphate Materials and TNX OD Background Sediment

\begin{tabular}{|c|c|c|c|c|c|c|c|c|c|c|}
\hline & Background $^{(\mathrm{a})}$ & $\begin{array}{c}\text { Proc.RP- } \\
\text { MT }\end{array}$ & $\begin{array}{l}\text { Proc. RP- } \\
\text { ID }\end{array}$ & $\begin{array}{l}\text { VP Proc. RP- } \\
\text { ID }\end{array}$ & PR-FL & $\begin{array}{c}\text { Brown Rock- } \\
\text { TN }\end{array}$ & $\begin{array}{c}\text { Washed P Ore- } \\
\text { TN }\end{array}$ & $\begin{array}{l}\text { WGI-PR- } \\
\text { ID }\end{array}$ & $\begin{array}{l}\text { Apatite- } \\
\text { NC }\end{array}$ & $\begin{array}{c}\text { Biological- } \\
\mathrm{P}\end{array}$ \\
\hline As & 1.5 & 11.1 & 10.2 & 10.0 & 3.7 & 4.8 & 2.3 & 10.5 & 3.8 & $<0.7$ \\
\hline $\mathrm{Ba}$ & 22 & 18.6 & 14.0 & 32.9 & 27.4 & 26.1 & 29.1 & 14.9 & 12.7 & 7.6 \\
\hline $\mathrm{Cd}$ & 24.8 & 0.4 & 24.0 & 1.8 & 1.7 & 0.2 & 0.2 & 43.0 & 17.9 & $<0.03$ \\
\hline Co & 0.7 & 0.4 & 0.9 & 67.6 & 2.1 & 2.8 & 3.9 & 1.0 & 0.3 & $<0.07$ \\
\hline $\mathrm{Cr}$ & 2.8 & 27.6 & 117.0 & 7.5 & 20.1 & 5.4 & 7.2 & 191.6 & 52.5 & 4.6 \\
\hline $\mathrm{Cu}$ & 2.2 & 2.7 & 34.3 & 89.4 & 3.4 & 5.0 & 4.7 & 27.5 & 3.9 & 3.3 \\
\hline $\mathrm{Fe}$ & 889 & 932.6 & 3338 & 6147 & 2410.4 & 5788 & 7973 & 1990 & 1555.5 & 41 \\
\hline M & 84 & 21.7 & 37.0 & 69.9 & 71.9 & 562.4 & 615.9 & 28.3 & 15.5 & 11.4 \\
\hline $\begin{array}{l}\mathrm{n} \\
\mathrm{M}\end{array}$ & $n a^{(b)}$ & 0.9 & 9.7 & 0.1 & 3.4 & 0.8 & 0.5 & 8.0 & 3.8 & 0.8 \\
\hline o & & & & & & & & & & \\
\hline $\mathrm{Ni}$ & na & 7.0 & 67.9 & 17.6 & 11.4 & 10.0 & 12.8 & 76.7 & 10.8 & $<0.7$ \\
\hline $\mathrm{Pb}$ & 12.2 & 11.5 & 5.1 & 21.0 & 6.3 & 4.2 & 5.7 & 2.9 & 3.1 & 1.3 \\
\hline $\mathrm{Sr}$ & na & 95.9 & 175.0 & 442.5 & 285.7 & 146.6 & 187.4 & 150.6 & 544.8 & 815.0 \\
\hline
\end{tabular}

Each phosphate sample was analyzed in two replicates

(a) Kaplan et al. (2002), sediment background values are for surface sediment collected just north of the operable unit.

(b) Not analyzed 
WSRC-TR-2003-00579, REV. 0

Table 4. Concentration $(\mathrm{mg} / \mathrm{kg}$ ) of Impurities in Tested Phosphate Sources

\begin{tabular}{lcccccc}
\hline & \multicolumn{2}{c}{ Processed } & \multicolumn{2}{c}{ Mined } & \multicolumn{2}{c}{ Biological } \\
\cline { 2 - 6 } & Avg & Stdev & Avg & Stdev & Avg & Stdev \\
\hline $\mathrm{As}$ & 10.4 & 1 & 5.1 & 0.3 & $<0.7$ & $\mathrm{NA}$ \\
$\mathrm{Ba}$ & 21.8 & 9 & 22.0 & 7.0 & 7.6 & 0.1 \\
$\mathrm{Cd}$ & 8.7 & 12 & 12.6 & 19.0 & $<0.03$ & $\mathrm{NA}$ \\
$\mathrm{Co}$ & 23.0 & 35 & 2.0 & 1.0 & $<0.07$ & $\mathrm{NA}$ \\
$\mathrm{Cr}$ & 50.7 & 54 & 55.4 & 80.0 & 4.6 & 0.0 \\
$\mathrm{Cu}$ & 42.1 & 39 & 8.9 & 10.0 & 3.3 & 1.5 \\
$\mathrm{Fe}$ & 3472.7 & 2346 & 3943.0 & 2672.0 & 41.0 & 0.2 \\
$\mathrm{Mn}$ & 42.9 & 22 & 258.8 & 290.0 & 11.4 & 0.2 \\
$\mathrm{Mo}$ & 3.6 & 5 & 3.3 & 3.0 & 0.8 & 0.1 \\
$\mathrm{Ni}$ & 30.8 & 29 & 24.3 & 30.0 & $<0.7$ & $\mathrm{NA}$ \\
$\mathrm{Pb}$ & 12.5 & 7 & 4.4 & 2.0 & 1.3 & 0.1 \\
$\mathrm{Sr}$ & 237.8 & 170 & 263.0 & 132.0 & 815.0 & 136.0 \\
\hline (a) & The number of observations for each mean varies; processed=6, mined=12, biological=2 \\
$\mathrm{NA}-$ not applicable & \multicolumn{5}{l}{}
\end{tabular}


Table 5. Total Concentration* ( $\mathrm{mg} / \mathrm{kg}$ ) of Impurities in Tested Phosphate Origin

\begin{tabular}{|c|c|c|c|c|c|c|c|c|c|c|c|c|}
\hline & \multicolumn{2}{|c|}{ Montana } & \multicolumn{2}{|c|}{ Idaho $^{\text {(a) }}$} & \multicolumn{2}{|c|}{ Tennessee $^{(b)}$} & \multicolumn{2}{|c|}{ North Carolina } & \multicolumn{2}{|c|}{ Florida } & \multicolumn{2}{|c|}{ Biological } \\
\hline & Avg & Stdev & Avg & Stdev & Avg & Stdev & Avg & Stdev & Avg & Stdev & Avg & Stdev \\
\hline$\overline{\text { As }}$ & 11.1 & 0.7 & 10.3 & 0.6 & 3.6 & 1.7 & 3.8 & NA & 3.7 & 0.6 & $<0.7$ & NA \\
\hline $\mathrm{Ba}$ & 18.6 & 0.0 & 20.6 & 9.7 & 27.6 & 2.7 & 12.7 & NA & 27.4 & 0.2 & 7.6 & 0.1 \\
\hline $\mathrm{Cd}$ & 0.4 & 0.0 & 22.9 & 18.8 & 0.2 & 0.0 & 17.9 & $\mathrm{NA}$ & 1.7 & 0.1 & $<0.03$ & NA \\
\hline $\mathrm{Co}$ & 0.4 & 0.0 & 23.2 & 34.5 & 3.4 & 0.9 & 0.3 & NA & 2.1 & 0.2 & $<0.07$ & NA \\
\hline $\mathrm{Cr}$ & 27.6 & 3.4 & 105.4 & 86.5 & 6.3 & 1.1 & 52.5 & NA & 20.1 & 0.1 & 4.6 & 0.0 \\
\hline $\mathrm{Cu}$ & 2.7 & 1.7 & 50.4 & 30.5 & 4.9 & 0.7 & 3.9 & NA & 3.4 & 0.8 & 3.3 & 1.5 \\
\hline $\mathrm{Fe}$ & 932.6 & 87.7 & 3824.9 & 1918.8 & 6880.4 & 1298.5 & 1555.5 & NA & 2410.4 & 109.9 & 40.9 & 0.2 \\
\hline $\mathrm{Mn}$ & 21.7 & 4.0 & 45.1 & 20.5 & 589.2 & 56.6 & 15.5 & NA & 71.9 & 0.8 & 11.4 & 0.2 \\
\hline Mo & 0.9 & 0.1 & 5.9 & 4.7 & 0.7 & 0.3 & 3.8 & NA & 3.4 & 0.3 & 0.8 & 0.1 \\
\hline $\mathrm{Ni}$ & 7.0 & 0.8 & 54.1 & 29.7 & 11.4 & 1.8 & 10.8 & NA & 11.4 & 0.4 & $<0.7$ & NA \\
\hline $\mathrm{Pb}$ & 11.5 & 1.8 & 9.7 & 8.9 & 5.0 & 1.1 & 3.1 & NA & 6.3 & 0.3 & 1.3 & 0.1 \\
\hline
\end{tabular}

* Each material was analyzed in two replicates

(a) Idaho = 3 materials: Proc. RP-ID, VP Proc. RP-ID, and WGI RP-ID

(b) Tennessee = Brown Rock-TN and Washed P Ore-TN

NA - not applicable 
WSRC-TR-2003-00579, REV. 0

Table 6. Concentration of Elements* $(\mathrm{mg} / \mathrm{kg})$ in TCLP Extract of Phosphate Sources

\begin{tabular}{lccccccc}
\hline Elements & $\begin{array}{c}\text { Regulatory } \\
\text { Level }^{(\mathrm{a})} \\
{[\mathrm{mg} / \mathrm{L}]}\end{array}$ & Avg & Stdev & Avg & Stdev & Avg & Stdev \\
\hline $\mathrm{As}$ & 5.0 & 1.1 & 0.9 & 1.0 & 0.7 & 0.8 & 0.0 \\
$\mathrm{Ba}$ & 100.0 & 2.1 & 1.7 & 1.0 & 0.5 & 0.3 & 0.0 \\
$\mathrm{Ca}$ & $\mathrm{NL}$ & 13333.4 & 7692.1 & 3708.6 & 2047.1 & 7620.0 & 145.2 \\
$\mathrm{Cd}$ & 1.0 & 1.3 & 1.0 & 0.9 & 2.0 & $<0.003$ & 0.0 \\
$\mathrm{Co}$ & $\mathrm{NL}$ & 19.5 & 33.5 & 0.1 & 0.2 & $<0.02$ & 0.0 \\
$\mathrm{Cr}$ & 5.0 & 0.5 & 0.5 & 0.1 & 0.0 & 0.1 & 0.0 \\
$\mathrm{Cu}$ & $\mathrm{NL}$ & 13.6 & 21.6 & 0.1 & 0.0 & 0.2 & 0.1 \\
$\mathrm{Fe}$ & $\mathrm{NL}$ & 58.0 & 94.5 & $<1.6$ & 0.0 & $<1.6$ & 0.0 \\
$\mathrm{Mg}$ & $\mathrm{NL}$ & 689.7 & 70.6 & 326.6 & 279.2 & 1592.5 & 48.8 \\
$\mathrm{Mn}$ & $\mathrm{NL}$ & 50.4 & 61.5 & 15.1 & 18.6 & 0.1 & 0.0 \\
$\mathrm{Mo}$ & $\mathrm{NL}$ & 0.5 & 0.4 & 9.4 & 20.7 & 0.1 & 0.0 \\
$\mathrm{Ni}$ & 70 & 8.1 & 11.2 & 2.1 & 3.2 & $<0.16$ & 0.0 \\
$\mathrm{~Pb}$ & 5.0 & 0.2 & 0.1 & $<0.12$ & 0.0 & $<0.12$ & 0.0 \\
$\mathrm{P}$ & $\mathrm{NL}$ & 1072.9 & 1752.5 & 168.0 & 139.4 & 3234.9 & 51.8 \\
$\mathrm{Sr}$ & $\mathrm{NL}$ & 22.3 & 13.5 & 21.7 & 31.8 & 63.2 & 1.5 \\
\hline
\end{tabular}

Each material was tested in two replicates.

(a) Maximum concentration of contaminants for toxicity characteristics (40 CFR 261.24, U.S. EPA, 1999)

(b) The number of observations for each mean varies; processed $=6$, mined $=12$, biological $=2$ NL - no RCRA limit 
WSRC-TR-2003-00579, REV. 0

Table 7. Concentration of Elements* $(\mathrm{mg} / \mathrm{kg})$ in TCLP Extract of Phosphate Origin

\begin{tabular}{|c|c|c|c|c|c|c|c|c|c|c|c|c|c|}
\hline \multirow[t]{2}{*}{ Elements } & \multirow{2}{*}{$\begin{array}{c}\text { Regulatory } \\
\text { Level } \\
{[\mathrm{mg} / \mathrm{L}]}\end{array}$} & \multicolumn{2}{|c|}{ Montana } & \multicolumn{2}{|c|}{ Idaho $^{\text {(a) }}$} & \multicolumn{2}{|c|}{ Tennessee $^{(b)}$} & \multicolumn{2}{|c|}{ North Carolina } & \multicolumn{2}{|c|}{ Florida } & \multicolumn{2}{|c|}{ Biological } \\
\hline & & Avg & Stdev & Avg & Stdev & Avg & Stdev & Avg & Stdev & Avg & Stdev & Avg & Stdev \\
\hline$\overline{\text { As }}$ & 5.0 & 1.20 & 0.19 & 1.21 & 0.96 & 1.60 & 0.00 & $<0.2$ & 0.00 & 0.25 & 0.08 & 0.84 & 0.03 \\
\hline $\mathrm{Ba}$ & 100.0 & 3.97 & 0.19 & 1.33 & 0.7 & 0.95 & 0.55 & 0.61 & 0.02 & 0.65 & 0.02 & 0.33 & 0.01 \\
\hline $\mathrm{Ca}$ & NL & 21041 & 548 & 7166 & 5538 & 2728 & 1608 & 7004 & 272 & 3544 & 431 & 7620 & 145 \\
\hline $\mathrm{Cd}$ & 1.0 & 0.15 & 0.01 & 2.73 & 1.6 & $<0.003$ & 0.00 & $<0.003$ & 0.00 & 0.02 & 0.00 & $<0.003$ & 0.00 \\
\hline $\mathrm{Co}$ & NL & 0.36 & 0.01 & 19.40 & 33.6 & $<0.02$ & 0.01 & $<0.02$ & 0.00 & 0.51 & 0.06 & $<0.02$ & 0.00 \\
\hline $\mathrm{Cr}$ & 5.0 & 1.04 & 0.05 & 0.22 & 0.2 & $<0.04$ & 0.00 & 0.05 & 0.00 & 0.05 & 0.00 & 0.13 & 0.00 \\
\hline $\mathrm{Cu}$ & NL & 2.23 & 0.15 & 12.92 & 22.2 & 0.14 & 0.03 & 0.08 & 0.00 & 0.13 & 0.00 & 0.17 & 0.05 \\
\hline $\mathrm{Fe}$ & NL & 167.11 & 7.73 & 2.83 & 2.1 & $<1.6$ & 0.00 & $<1.6$ & 0.00 & 0.81 & 0.74 & $<1.6$ & 0.00 \\
\hline $\mathrm{Mg}$ & NL & 655.88 & 3.07 & 679.40 & 79.6 & 51.18 & 70.12 & 321.75 & 19.44 & 583.79 & 68.01 & 1592.51 & 48.80 \\
\hline $\mathrm{Mn}$ & NL & 26.85 & 0.78 & 41.86 & 67.8 & 34.08 & 13.10 & 1.17 & 0.01 & 4.68 & 0.43 & 0.12 & 0.00 \\
\hline Mo & NL & 0.59 & 0.04 & 0.35 & 0.5 & 23.26 & 32.81 & 0.05 & 0.00 & 0.12 & 0.01 & 0.06 & 0.01 \\
\hline $\mathrm{Ni}$ & 70 & 0.82 & 0.08 & 10.36 & 9.6 & 0.18 & 0.03 & 0.71 & 0.06 & 1.81 & 0.23 & $<0.16$ & 0.00 \\
\hline $\mathrm{Pb}$ & 5.0 & 0.32 & 0.05 & 0.15 & 0.1 & $<0.12$ & 0.00 & $<0.12$ & 0.00 & $<0.12$ & 0.00 & $<0.12$ & 0.00 \\
\hline $\mathrm{P}$ & NL & 90.32 & 1.03 & 1081.56 & 1745.3 & 100.81 & 56.55 & 110.30 & 5.81 & 411.83 & 29.04 & 3234.94 & 51.79 \\
\hline $\mathrm{Sr}$ & NL & 31.11 & 0.16 & 14.76 & 12.4 & 5.28 & 3.44 & 78.32 & 3.45 & 11.22 & 1.36 & 63.15 & 1.48 \\
\hline
\end{tabular}

Each material was tested in two replicates.

(a) Idaho = 3 materials: Proc. RP-ID, VP Proc. RP-ID, \& WGI RP-ID; 2 replicates of each material

(b) Tennessee $=$ Brown Rock-TN and Washed P Ore-TN; 2 replicates of each material

$\mathrm{NL}$ - no RCRA limit 
WSRC-TR-2003-00579, REV. 0

Table 8. Solubility of Phosphate Materials in Comparison with Some Phosphate Mineral Phases; the Solubility was Calculated by Following Davies Equation (Sposito, 1986)

\begin{tabular}{|lcclr|}
\hline $\begin{array}{l}\text { Phosphate } \\
\text { Materials }\end{array}$ & $\begin{array}{c}\text { pH } \\
\text { Processed }\end{array}$ & Avg $\log (\mathrm{Ksp})$ & Mineral Phase & \multicolumn{1}{c|}{$\begin{array}{c}\text { Log } \\
(\mathrm{Ksp})\end{array}$} \\
Mined & 7.40 & -48.0 & $\mathrm{~Pb} 5(\mathrm{PO} 4) 3(\mathrm{OH}, \mathrm{Cl})$ & -76.5 \\
Biological & 7.10 & -57.0 & $\mathrm{Sr} 5(\mathrm{PO} 4) 3(\mathrm{OH})$ & -51.3 \\
& & -45.2 & $\mathrm{Zn3}(\mathrm{PO} 4) 3$ & -35.3 \\
& & $\mathrm{Cd} 3(\mathrm{PO} 4) 3$ & -32.6 \\
& & Pu(PO4) & -24.4 \\
& & & Quartz $(\mathrm{SiO} 2)$ & -4 \\
\hline Davies Eq.: & & & \\
$\log (\mathrm{Ksp})=10 \log (\mathrm{Ca})+6 \log \left(\mathrm{PO}_{4}{ }^{3-}\right)+2 \log \left(\mathrm{OH}^{-}\right)$ & & \\
\hline
\end{tabular}

Table 9. Concentration of Elements* $(\mathrm{mg} / \mathrm{kg})$ in Water Soluble Fraction of Phosphate Materials

\begin{tabular}{lcccccc}
\hline Elements & \multicolumn{2}{c}{ Processed $^{(\mathrm{a})}$} & \multicolumn{2}{c}{ Mined } & \multicolumn{2}{c}{ Biological } \\
$(\mathrm{mg} / \mathrm{kg})$ & $\mathrm{Avg}$ & Stdev & Avg & Stdev & Avg & Stdev \\
\hline $\mathrm{As}$ & 1.02 & 0.98 & 0.06 & 0.04 & 0.32 & 0.01 \\
$\mathrm{Ba}$ & 0.34 & 0.27 & 0.10 & 0.12 & 0.01 & 0.00 \\
$\mathrm{Ca}$ & 1593.18 & 1111.11 & 185.31 & 137.15 & 278.70 & 38.76 \\
$\mathrm{Cd}$ & 0.25 & 0.20 & 0.08 & 0.17 & 0.00 & 0.00 \\
$\mathrm{Co}$ & 9.50 & 14.58 & 0.01 & 0.01 & 0.00 & 0.00 \\
$\mathrm{Cr}$ & 1.03 & 1.05 & 0.55 & 1.18 & 0.04 & 0.02 \\
$\mathrm{Cu}$ & 6.41 & 9.63 & 0.06 & 0.11 & 0.02 & 0.00 \\
$\mathrm{Fe}$ & 57.99 & 72.17 & 6.67 & 8.09 & 0.38 & 0.04 \\
$\mathrm{Mg}$ & 196.19 & 136.71 & 29.88 & 32.38 & 200.52 & 14.34 \\
$\mathrm{Mn}$ & 16.13 & 24.11 & 0.53 & 0.73 & 0.01 & 0.00 \\
$\mathrm{Mo}$ & 0.19 & 0.14 & 0.15 & 0.18 & 0.04 & 0.01 \\
$\mathrm{Ni}$ & 3.78 & 4.60 & 0.45 & 0.86 & 0.05 & 0.01 \\
$\mathrm{~Pb}$ & 0.10 & 0.05 & 0.03 & 0.01 & 0.03 & 0.00 \\
$\mathrm{Sr}$ & 4.52 & 3.54 & 0.93 & 1.37 & 2.56 & 0.35 \\
$\mathrm{P}$ & 307.31 & 458.27 & 20.20 & 26.41 & 504.81 & 77.09 \\
\hline * Each material was tested in three replicates. \\
(a) The number of observations for each mean varies; processed=9, mined=18, biological=3
\end{tabular}


WSRC-TR-2003-00579, REV. 0

\subsection{EFFECTIVE DIFFUSION COEFFICIENTS CALCULATED FROM KD VALUES}

The objective of the phosphate and ferrous iron sorption isotherm experiments was to measure the phosphate or iron distribution coefficient, $\mathrm{K}_{\mathrm{d}}$, in the TNX OD sediment (B5) (Figure 2) to permit calculation of an effective diffusion coefficient, $D_{\text {eff, }}$ using equations 1 , 2 , and 3. These values are presented in Table 10. The $K_{d}$ based $D_{\text {eff }}$ values were in the order of $10^{-8} \mathrm{~cm}^{2} / \mathrm{s}$ and provide a theoretical estimate based on a number of chemical assumptions (no precipitation, reversible adsorption/desorption, linear isotherm behavior, etc...) (Table $10)$.
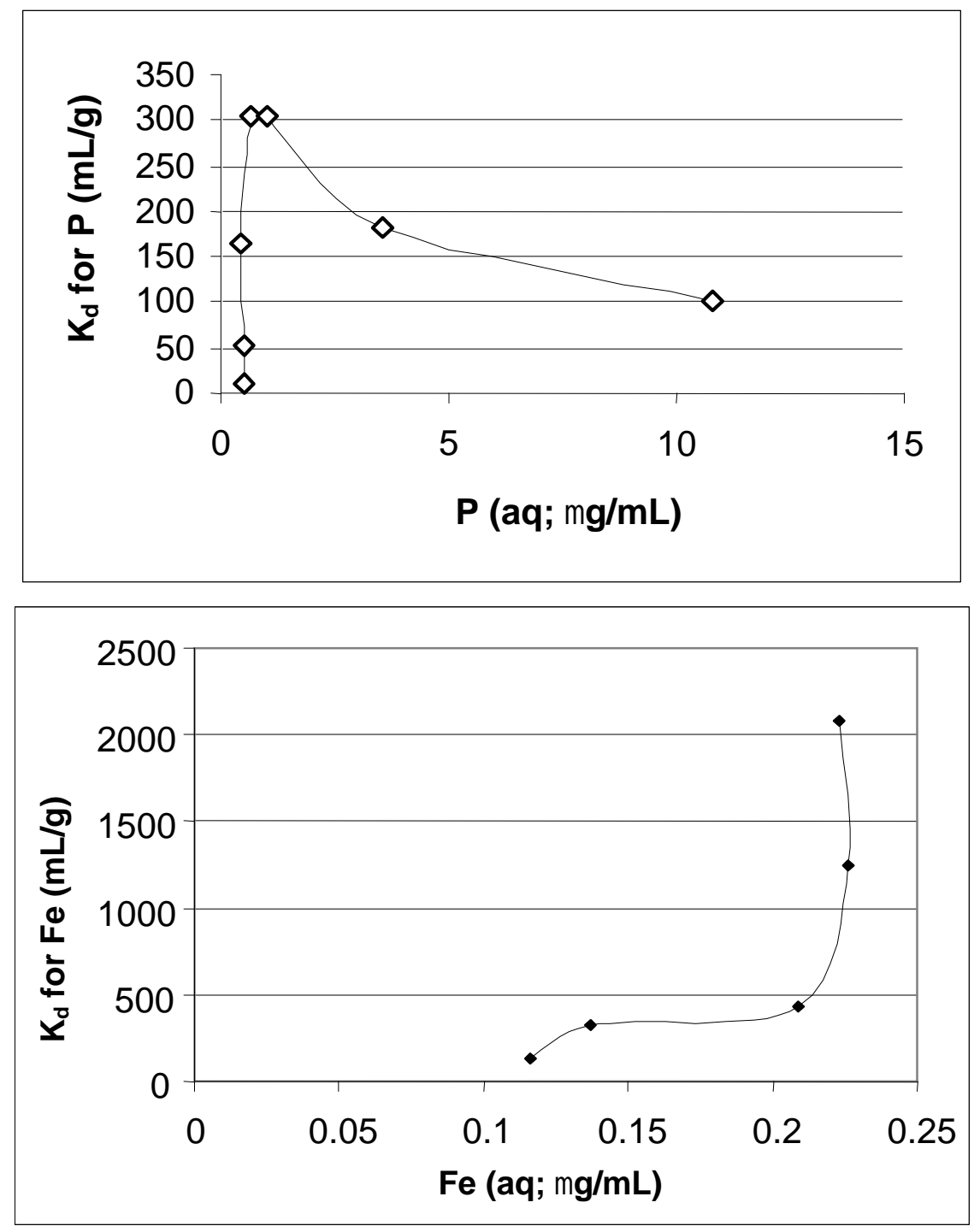

Figure 2. The Distribution Coefficients $\left(\mathrm{K}_{\mathrm{d}}\right)$ for $\mathrm{P}$ and $\mathrm{Fe}$ for the Sediment (B5) from the TNX OD 
WSRC-TR-2003-00579, REV. 0

Table 10. Effective Diffusion Coefficients $\left(\mathrm{cm}^{2} / \mathrm{s}\right)$ Calculated from Field Data, Laboratory Data, and $\mathrm{K}_{\mathrm{d}}$ Values

\begin{tabular}{|c|c|c|c|}
\hline & Field $^{(a)}$ & Laboratory $^{(a)}$ & Calculated from $\mathrm{K}_{\mathrm{d}}^{(\mathrm{b})}$ \\
\hline Biological Apatite & NA & $e-6$ & e-8 \\
\hline $\mathrm{NCA}$ & 6.71 & & \\
\hline $\mathrm{Fe}(0)$ & $1.78 \mathrm{e}$ & & \\
\hline \multicolumn{4}{|c|}{$\begin{array}{l}\text { (a) Calculated from equation } 1 \text { and } 2 . \\
\text { (b) Calculated from equations 3: assuming Fe } \mathrm{Kd}=200 \mathrm{~mL} / \mathrm{g} \text { and P Kd }=125 \mathrm{~mL} / \mathrm{g} \text { from } \\
\text { Figure 2, porosity }=0.4 \text {, particle density }=1.65 \mathrm{~g} / \mathrm{cm}^{3}, \mathrm{Fe} \& \mathrm{P} \text { diffusion in water }=1 \mathrm{e}-5 \mathrm{~cm}^{2} / \mathrm{s} \\
\text { from Dragun, } 1998 \text { and Hillel, } 1980 . \\
\text { NA - not analyzed }\end{array}$} \\
\hline
\end{tabular}

Figure 2 presents $K_{d}$ values as a function of aqueous $P$ and Fe concentrations. These types of graphs are referred to as sorption isotherms and can provide indirect evidence regarding how a solute, in this case $\mathrm{P}$ or $\mathrm{Fe}$, sorb to the solid phase. At low aqueous $\mathrm{P}$ concentrations, there is a steep increase in $K_{d}$ values, followed by a gradual decline. This is a common isotherm pattern for solutes that sorb strongly to a sediment, but once the "high energy" sorption sites are filled, the proportion of solid to aqueous phase $\mathrm{P}$ concentrations gradually decrease generally in a linear manner with respect to aqueous $\mathrm{P}$ concentrations.

The sorption isotherm for Fe was quite different from that for $\mathrm{P}$ (Figure 2). At low concentrations, the $\mathrm{Fe}$ gradually sorbed onto the sediment, but after a critical point, $\sim 0.21$ $\mu \mathrm{g} / \mathrm{mL}$, the $\mathrm{K}_{\mathrm{d}}$ value increases sharply. This isotherm pattern is a classic example of a moderately strong sorbing solute precipitating out of solution once solubility is exceeded.

This type of data underscores the importance of using isotherm data, as compared to single sorption values, such as a $K_{d}$ value, to represent solute geochemical behavior in sediments. However, in the case of $\mathrm{Fe}$, solubility maintains the aqueous $\mathrm{Fe}$ concentrations to $<0.2$ $\mu \mathrm{g} / \mathrm{mL}$, thereby greatly limiting the range of possible $\mathrm{K}_{\mathrm{d}}$ values. Thus, a combination of both solubility controls and $\mathrm{K}_{\mathrm{d}}$ values may be best for describing Fe geochemistry in this system. In the case of $\mathrm{P}$, solubility does not appear to have been exceeded and the apparent $\mathrm{K}_{\mathrm{d}}$ values varied greatly over the range of aqueous $\mathrm{P}$ concentrations evaluated. Sediment porewater $\mathrm{P}$ concentrations at TNX OD are $<1 \mathrm{mg} / \mathrm{L}$, and as such, are expected to sorb to the sediment in greatly varying degrees, depending on slight changes in the aqueous concentrations. 
WSRC-TR-2003-00579, REV. 0

\subsection{ZONE-OF-INFLUENCE (ZOI) OF AMENDMENTS UNDER LABORATORY CONDITIONS}

The laboratory experiment was conducted under controlled conditions in a $60 \mathrm{~mL}$ syringe at $21^{\circ} \mathrm{C}$. As such, they isolate the diffusion process from other water transport processes that may occur under natural field conditions, such as advection, colloidal transport, and macropore flow. In the laboratory study, diffusion of $\mathrm{P}$ and $\mathrm{Fe}$ was determined by the measurement of these element concentrations in sediment B5, which was in contact with North Carolina Apatite, biological $\mathrm{P}$ or zero-valent iron, $[\mathrm{Fe}(0)]$ for three months. The data for the biological $\mathrm{P}$ and North Carolina apatite are presented in Figure 3. These concentrations were then used to calculate effective diffusion coefficients $\left(\mathrm{D}_{\text {eff }}\right)\left(\right.$ Table 10). The estimated Fe and $P D_{\text {eff }}$ values were similar, in the order of $10^{-6} \mathrm{~cm}^{2} / \mathrm{s}$ (Table 10).

Diffusion is a spontaneous process that results in the movement of a solute. Diffusion is caused by the random thermal motion of a solute in solution and is driven by concentration gradients. Solutes move from high concentration areas to low concentration areas. To provide a measure of lateral movement of the $\mathrm{P}$ and $\mathrm{Fe}$ in the vertical treatments, laboratory data were collected and applied to diffusion equations (Fick's second law and equation 3) to provide effective diffusion coefficients.

The background P concentration in B5 sediment was $318 \mathrm{mg} / \mathrm{kg}$ (Table 11). In the treatment with biological apatite, the $\mathrm{P}$ concentration was the highest in the first slice of the sediment (a distance from apatite of 0 to $0.4 \mathrm{~cm}$ ), $5484 \mathrm{mg} / \mathrm{kg}$ (Table 11). The P concentration in the second slice was drastically lower than in the fist slice but still higher than the $\mathrm{P}$ background concentration (Figure 3). In remaining three further slices, i.e., the distance from $0.9 \mathrm{~cm}$ to $2.9 \mathrm{~cm}, \mathrm{P}$ concentrations remained higher than the $\mathrm{P}$ background concentration for this sediment. In the treatment with $\mathrm{NC}$ apatite, the $\mathrm{P}$ concentration was the highest in the first two slices of the sediment; however, it was considerably lower than in the treatment with biological apatite (Figure 4 and Table 11). In the treatments with metallic iron, in a period of three months, Fe traveled the distance of less than $1.0 \mathrm{~cm}$, and the highest concentration of Fe was observed in the first slice, $15095 \mathrm{mg} / \mathrm{kg}$ (Figure 4 and Table 11). 
WSRC-TR-2003-00579, REV. 0

Table 11. Diffusion of $\mathrm{P}$ or Fe in Treatments with Phosphate Source or Fe(0); Laboratory Experiment, Average Values Based on Two Replicates

\begin{tabular}{lrrrrr}
\hline \multicolumn{5}{c}{ Diffusion of P (P concentration in mg/kg) } \\
\cline { 2 - 6 } Treatments & 0.5 & 1 & 1.5 & 2 & 2.5 \\
Background (B5) avg & 318 & 318 & 318 & 318 & 318 \\
Background (B5) stdev & 7.6 & 7.6 & 7.6 & 7.6 & 7.6 \\
Biological-P avg & 5484 & 1386 & 1054 & 849 & 640 \\
Biological-P stdev & 2183 & 167 & 30 & 30 & 11 \\
Ncapatite avg & 440 & 460 & 388 & 372 & 386 \\
NCapatite stdev & 47 & 174 & 88 & 2 & 40 \\
\hline \multicolumn{5}{c}{ Diffusion of Fe (Fe concentration in mg/kg) } \\
\hline \multicolumn{5}{c}{ Distance (cm) from Fe source } \\
Treatments & 0.5 & 1 & 1.5 & 2 & 2.5 \\
Background (B5) avg & 12052 & 12052 & 12052 & 12052 & 12052 \\
Background (B5) stdev & 1248 & 1248 & 1248 & 1248 & 1248 \\
Fe(0) avg & 15095 & 11402 & 11241.5 & 11770.5 & 12229 \\
Fe(0) stdev & 3855 & 2427 & 313 & 455 & 434 \\
\hline
\end{tabular}


WSRC-TR-2003-00579, REV. 0
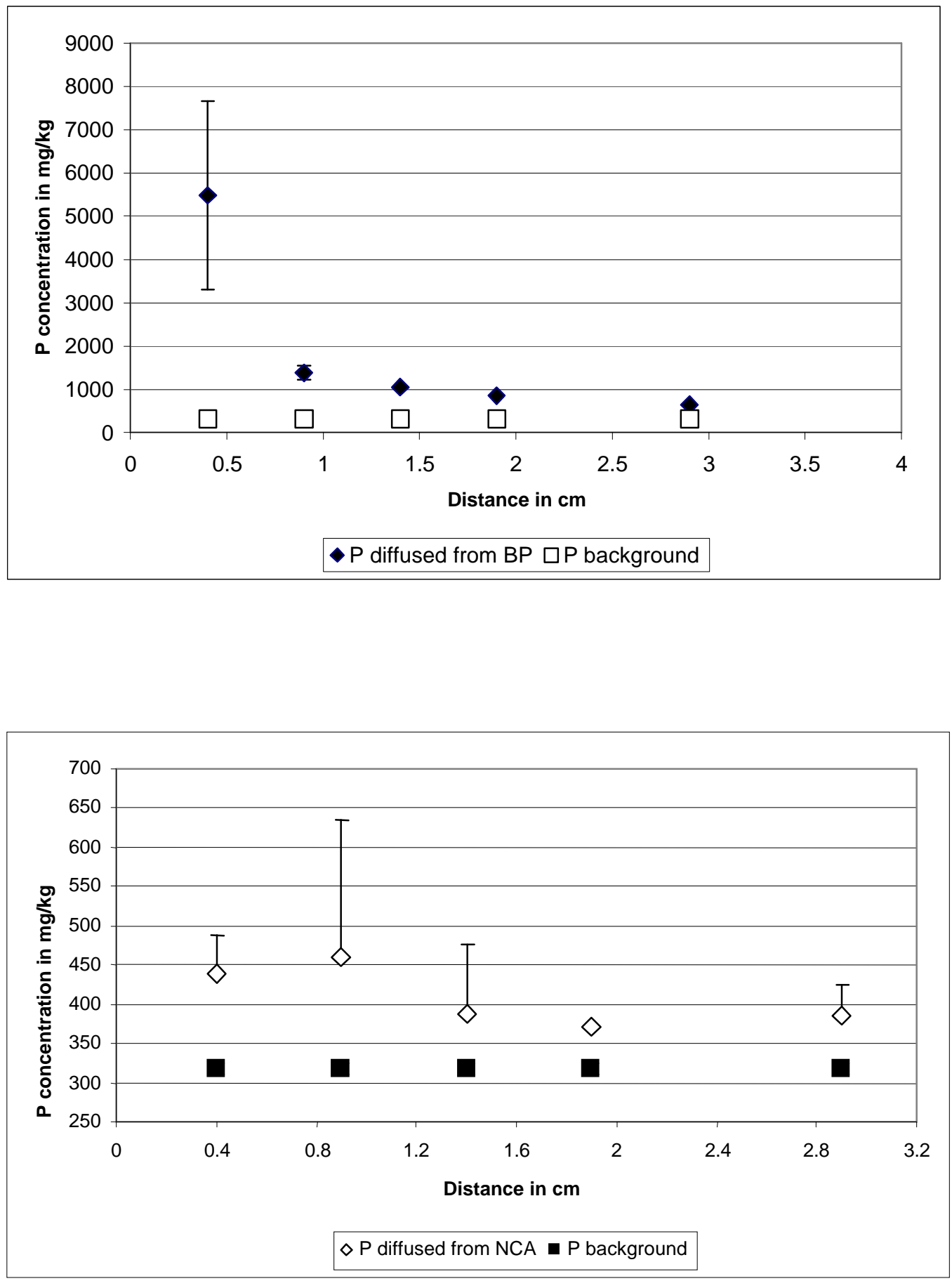

Figure 3. Profile of P Diffusion in the B5 Sediment from the TNX OD after 3 Months of Contact with Biological P (BP) and North Carolina (NCA): Laboratory Study 
WSRC-TR-2003-00579, REV. 0

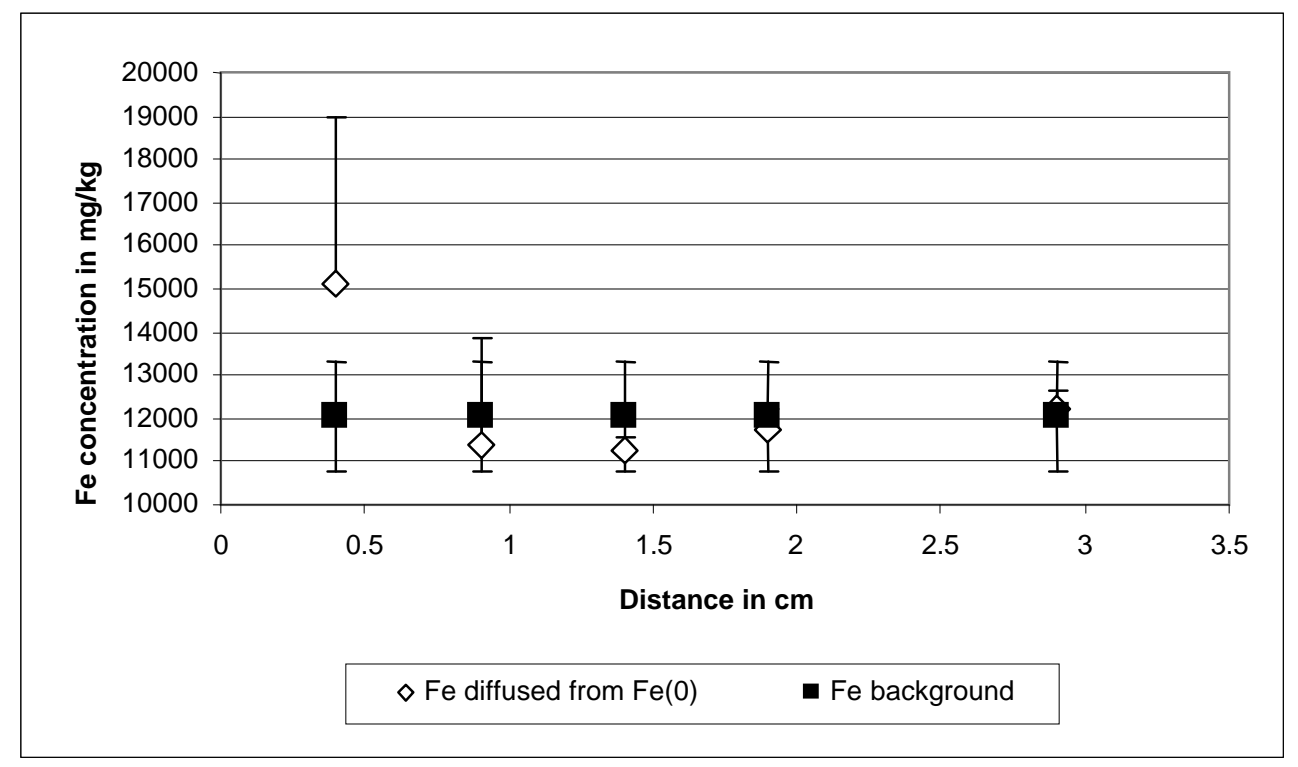

Figure 4. Profile of Fe Diffusion in the B5 Sediment from the TNX OD after 3 Months of Contact with $\mathrm{Fe}(0)$ : Laboratory Study

\subsection{ZONE-OF-INFLUENCE (ZOI) OF AMENDMENTS UNDER FIELD CONDITIONS}

In the horizontal treatments five amendments (North Carolina Apatite, biological apatite, Fe $(0)$, and $\mathrm{F}(0)$ mixed with North Carolina Apatite and $\mathrm{Fe}(0)$ mixed with biological apatite) were spread on the surface of the sediment. For the treatments with P source, i.e., apatite minerals or apatite mixed with $\mathrm{Fe}(0)$, the transport of $\mathrm{P}$ as a function of depth was evaluated. The transport of $\mathrm{P}$ in these treatments occurred very likely by saturated flow, involving diffusion and advection. The background average concentration of $\mathrm{P}$ in the sediment top layer (zero distance from $\mathrm{PO}_{4}{ }^{3-}$ source) was $1057 \mathrm{mg} / \mathrm{kg}$ and it decreased to less than 900 $\mathrm{mg} / \mathrm{kg}$ in the last two bottom layers tested, i.e., depth from 3 to $5 \mathrm{~cm}$ (Figure 5 and Table 8C). Addition of North Carolina Apatite and biological apatite significantly increased the $P$ concentrations in the two first layers (i.e., depth from 0 to $2 \mathrm{~cm}$ ) up to 7030 and $7634 \mathrm{mg} / \mathrm{kg}$, respectively, in plots with North Carolina Apatite and biological apatite (Figure 5). In depths from $2 \mathrm{~cm}$ to $5 \mathrm{~cm}$, $\mathrm{P}$ concentrations in plots with both types of apatite remained similar to the background $\mathrm{P}$ concentrations.

Concurrent applications of apatite (North Carolina or biological) and $\mathrm{Fe}(0)$ did not induce $\mathrm{P}$ or Fe transport through the sediment profile. The $\mathrm{P}$ and Fe concentration distribution in the sediment in these treatments was similar to the $\mathrm{P}$ and Fe distribution in the background sediment (Figure 5). Phosphate has been reported to be chemically associated with $\mathrm{Fe}$ in soils or sediments (Wang et al., 1991). Very likely in the presence of high content of $\mathrm{PO}_{4}{ }^{3-}$ and $\mathrm{Fe}$, under reducing conditions ferrous phosphates, e.g., vivianite $\left[\mathrm{Fe}_{3}\left(\mathrm{PO}_{4}\right)_{2}{ }^{\cdot} 8 \mathrm{H}_{2} \mathrm{O}\right]$ precipitated. In the treatment where only $\mathrm{Fe}(0)$ was added, Fe traveled to the depth of $5 \mathrm{~cm}$; however, the highest $\mathrm{Fe}$ concentrations were observed in the first three layers, i.e., to the depth of $3 \mathrm{~cm}$ (Figure 5). 
In the vertical treatments (lateral distance) where the amendments were added to a hole 15 $\mathrm{cm}$ deep, the transport of elements of interest, i.e., $\mathrm{P}$ and Fe, was based only on saturated diffusion. The analyzed distance from the source of $P$ or Fe was $3 \mathrm{~cm}$. The distance of $3 \mathrm{~cm}$ was divided into three strips $(1 \mathrm{~cm}$ wide) and data for each strip are presented in Figure 6.

The diffusion of $\mathrm{P}$ from both sources of $\mathrm{P}$, i.e., North Carolina and biological apatite, were almost identical (Figure 6). Phosphorous in these two treatments traveled 2 or $2.5 \mathrm{~cm}$. The background Fe concentration in the tested site sediment was $6101 \mathrm{mg} / \mathrm{kg}$, applied $\mathrm{Fe}(0)$ increased $\mathrm{Fe}$ concentrations in all tested strips of the sediment and data showed that $\mathrm{Fe}$ diffused though the longer distance than P (Figure 6).

Effective diffusion coefficients based on the field data were in the order of $10^{-6} \mathrm{~cm}^{2} / \mathrm{s}$, values very similar to those derived from the laboratory study (Table 10). The two order of magnitude greater rate than the theoretical value based on $K_{d}$ values likely indicates that the underlying assumptions based on the theoretical values are inappropriate. 
WSRC-TR-2003-00579, REV. 0
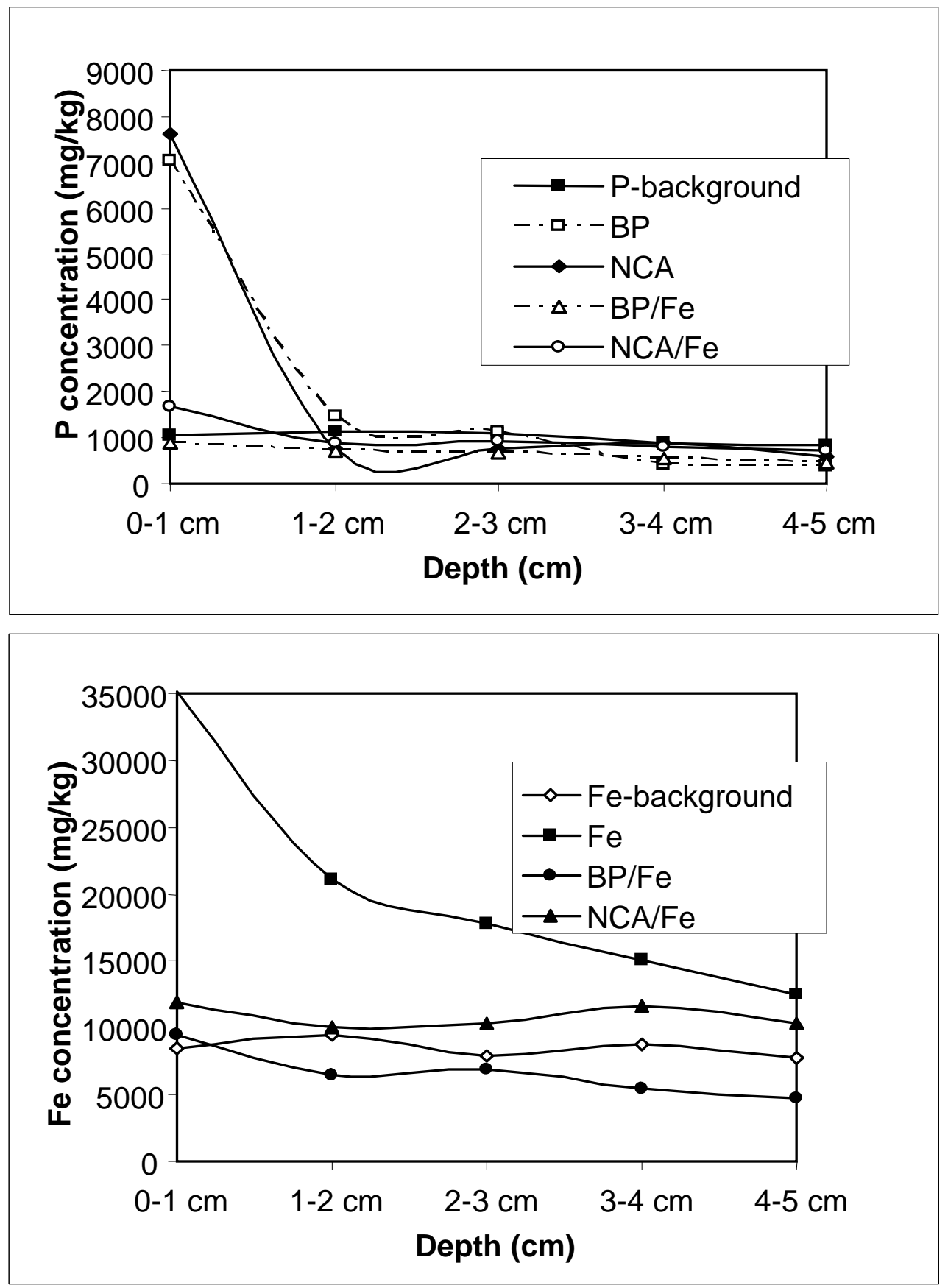

Figure 5. Transport of $\mathrm{P}$ and $\mathrm{Fe}$ in the Horizontal Treatments; the Total Evaluated Depth from the Element Source was $5 \mathrm{~cm}$ 
WSRC-TR-2003-00579, REV. 0
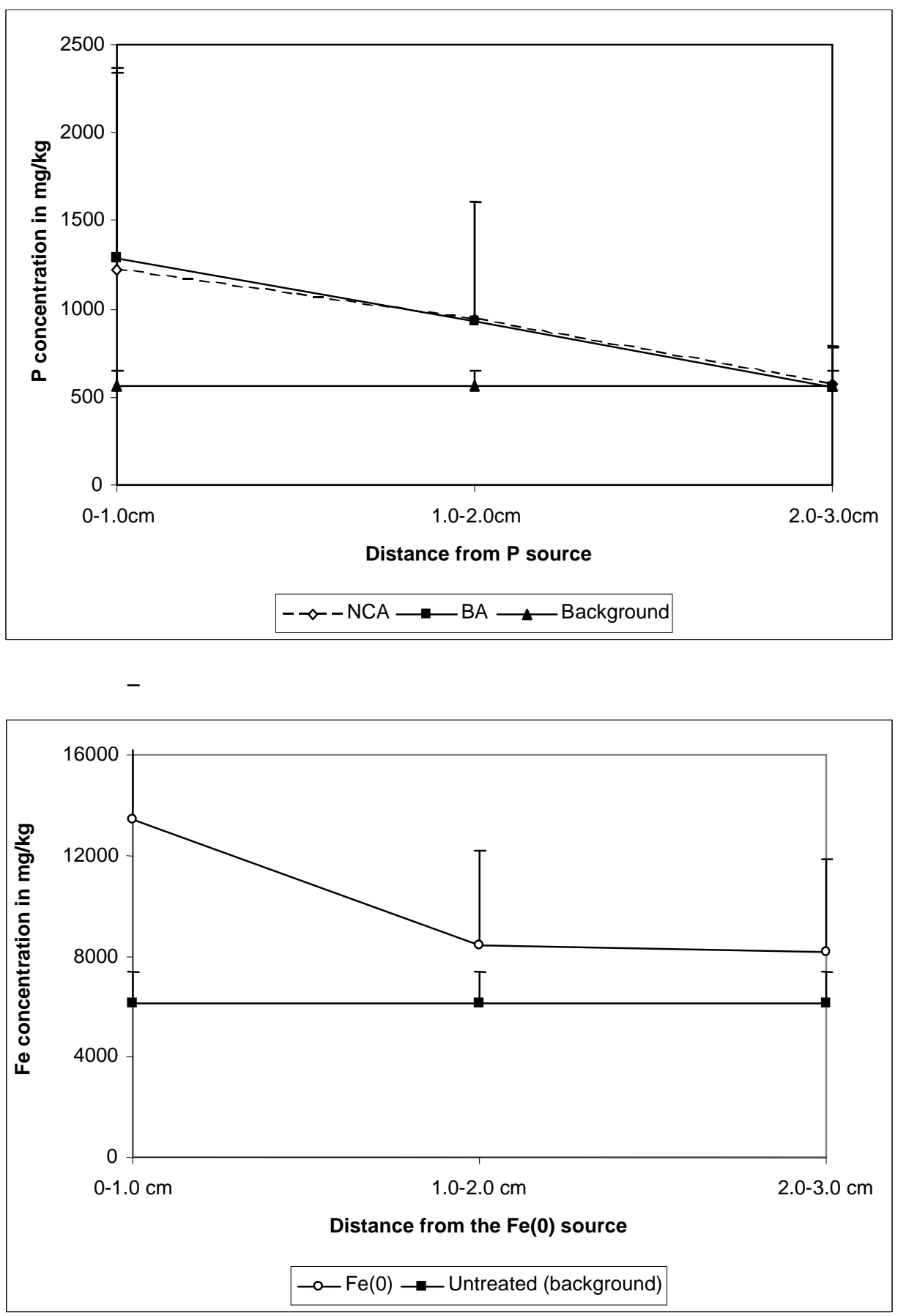

Figure 6. Transport of P and Fe in the Vertical Treatments; the Evaluated Distance for Element was $3 \mathrm{~cm}$ 
WSRC-TR-2003-00579, REV. 0

\subsection{PHOSPHORUS AND IRON DIFFUSION MODELING}

Sediment $\mathrm{P}$ and $\mathrm{Fe}$ concentration data were obtained from the laboratory and field study for calculations of $\mathrm{P}$ and $\mathrm{Fe}(0)$ diffusion. Metallic iron, biological $\mathrm{P}$ and North Carolina apatite were used in those tests. Utilizing PORFLOWTM software, the diffusion coefficients for $\mathrm{P}$ and $\mathrm{F}(0)$ were estimated based on the test data.

PORFLOWTM is developed and marketed by Analytic \& Computational Research, Inc. to solve problems involving transient and steady-state fluid flow, heat and mass transport in multi-phase, variably saturated, porous or fractured media with dynamic phase change. The porous/fractured media may be anisotropic, arbitrary heterogeneous sources (injection or pumping wells) may be present and, chemical reactions or radioactive decay may take place. PORFLOWTM has been widely used in the DOE complex to address major issues related to groundwater and nuclear waste management. PC-based PORFLOWTM Version 4.0 (dated 14 September 2000) was used in this work.

To calculate $\mathrm{P}$ and $\mathrm{Fe}(0)$ diffusion, PORFLOWTM models were set up to reflect the initial test conditions. The models were run to simulate both the laboratory and field tests. Note that the concentration data were obtained from the 91-day ( 3 months) laboratory experiments and from the 395-day (13 months) field study. Effective diffusion coefficients are optimized so that the modeling results would closely agree with the test data.

Table 12 lists the optimal effective diffusion coefficients of $\mathrm{Fe}(0)$, biological $\mathrm{P}$, and $\mathrm{NC}$ apatite for the laboratory and field studies. Overall, except for biological P, the diffusion coefficients for both studies are in agreement within one order of magnitude. The biological P data strongly suggests that precipitation occurred, a process that was not simulated in our PORFLOWTM models.

Using the optimal effective diffusion coefficients obtained from the field study, the radius distance that $\mathrm{Fe}$ and $\mathrm{P}$ diffused away from an infinite source term was calculated as a function of time. The calculated aqueous $\mathrm{P}$ or Fe concentration used to set the outer limit of diffusion was $1 \mathrm{E}^{-7} \mathrm{M}$ above background levels (note: the computer calculates concentrations $<1 \mathrm{E}^{-21} \mathrm{M}$, such concentrations would have no impact on sequestration, the phenomena of interest). For biological and NC apatite, an effective diffusion coefficient of $1.865 \mathrm{E}^{-6} \mathrm{~cm}^{2} / \mathrm{s}$ (i.e., average of the two estimated diffusion coefficients) was used. The results are summarized in Table 12. Similar distance calculations were not conducted with the laboratory-derived diffusion coefficients because of their similarity with the field-derived values. 
WSRC-TR-2003-00579, REV. 0

Table 12. Calculated P and Fe Effective Diffusion Coefficients (Deff) Based on Laboratory and Field study. Calculated Radius of Sediment Amendment Zone-of-Influence Based on Field Study

\begin{tabular}{|c|c|c|c|c|c|c|}
\hline \multirow[b]{3}{*}{ Amendment } & \multirow{3}{*}{$\begin{array}{l}\text { Laboratory } \\
\qquad \begin{array}{c}D_{\text {eff }} \\
\left(\mathrm{cm}^{2} / \mathrm{s}\right)\end{array}\end{array}$} & \multicolumn{5}{|c|}{ Field } \\
\hline & & \multirow{2}{*}{$\begin{array}{c}D_{\text {eff }} \\
\left(\mathrm{cm}^{2} / \mathrm{s}\right)\end{array}$} & \multicolumn{4}{|c|}{$\begin{array}{c}\text { Radius Distance P or Fe Travels in T-Area Sediment } \\
(\mathrm{cm})\end{array}$} \\
\hline & & & 13 months & $5 \mathrm{yr}$ & $7 \mathrm{yr}$ & $10 \mathrm{yr}$ \\
\hline $\mathrm{Fe}(0)$ & $1.77 \mathrm{E}^{-6}$ & $2.9 \mathrm{E}^{-6}$ & 2.1 & 4.5 & 5.2 & 6.3 \\
\hline Biological P & $3.0 \mathrm{E}^{-7}$ & $1.76 \mathrm{E}^{-6}$ & 2.3 & 4.9 & 5.8 & 6.9 \\
\hline NC Apatite & $6.7 \mathrm{E}^{-6}$ & $1.97 \mathrm{E}^{-6}$ & 1.8 & 3.9 & 4.6 & 5.5 \\
\hline
\end{tabular}

${ }^{\dagger}$ : Determined at concentration of $1 \mathrm{E}^{-7} \mathrm{~mol} / \mathrm{L}$. For Biological $\mathrm{P}$ and NC Apatite, average $\mathrm{D}_{\text {eff }}$ of $1.865 \mathrm{E}^{-6} \mathrm{~cm}^{2} / \mathrm{s}$ was used. 


\section{CONCLUSIONS}

Nine commercially available phosphate minerals were evaluated for solubility, concentration of trace metals, and leachability of trace metals. Processed and mined rock phosphate contain high total concentrations of $\mathrm{As}, \mathrm{Co}, \mathrm{Cr}, \mathrm{Cu}$, and $\mathrm{Sr}$, however, they did not exceed the RCRA TCLP limits. The use of stronger extractants indicated that these elements were very strongly bound by most apatite material; therefore, if they were applied to contaminated sediment at a rate sufficient for remediation, they would not pose an environmental risk. The biogenic apatite (fish bone) contained significantly lower metal impurities than processed and mined rock phosphate and was appreciably more soluble, i.e., it had a $\log \mathrm{K}_{\mathrm{sp}}$ of -45.2 compared to -57.0 for the mined rock phosphate samples.

The ZOI of surface applied amendments (horizontally applied), and amendments backfilled into holes (vertically applied) were evaluated under field conditions. Additionally, vertical application of ZOI was tested in the lab. For the surface applied amendments in the13-month field study, P from the biological apatite and from the mined apatite moved $\sim 3 \mathrm{~cm}$; and Fe from the $\mathrm{Fe}(0)$ treatment moved $\sim 5 \mathrm{~cm}$. Retardation factors were calculated and used to estimate the zone-of-influence at various intervals. To provide a measure of lateral movement of the $\mathrm{P}$ and Fe in the vertical treatments, field and laboratory data were collected and applied to diffusion equations (Fick's second law) to provide effective diffusion coefficients. Both sets of data were reasonably consistent, indicating that $\mathrm{P}$ from the biological and mined apatite and $\mathrm{Fe}$ from $\mathrm{Fe}(0)$ would be expected to diffuse more than $5 \mathrm{~cm}$ after 10 years. Both downward and lateral movement of $\mathrm{P}$ and $\mathrm{Fe}$ were greatly reduced when $\mathrm{Fe}(0)$ and apatite were applied concurrently. Based on thermodynamic calculations, an iron phosphate phase precipitated from solution, reducing the migration of each mineral, thereby greatly reducing the zone-of-influence.

In summary, there are several sources of phosphate that may be safely and effectively applied to the site. By mixing biogenic with mined phosphate, it will be possible to obtain a wide range of phosphate solubility, permitting the rapid immobilization of contaminants, while at the same time providing a slow release of phosphate for continued sediment treatment. Both materials are inexpensive and locally available: biological phosphate from Florida (perhaps South Carolina) and mined phosphate from North Carolina. The zone-of-influence for these amendments will increase relatively slowly. It will not be possible to apply $\mathrm{Fe}(0)$ along with apatite to the site and create a large zone-of-influence because an iron-phosphate phase precipitates, greatly reducing the mobility, and therefore the zone-of-influence of iron and phosphate. If both amendments are deemed necessary, it would be best to apply them at different times.

\section{ACKNOWLEDGEMENTS}

We appreciate the technical assistance provided by Cathy Coffey (WSRC). 
WSRC-TR-2003-00579, REV. 0

\section{REFERENCES}

Dragun, J. 1998. The Sediment Chemistry of Hazardous Materials, $2^{\text {nd }}$ edition. Amherst Scientific Publisher, Amherst, MA.

Eary, L. E., and D. Rai. 1987. Kinetics of Chromium(III) Oxidation to Chromium(VI) by Reaction with Manganese Dioxide. 21:1187-1193.

Hillel, D. 1980. Fundamentals of Sediment Physics. Academic Press. New York.

Kaplan, D., A.S. Knox, and C. Coffey. 2002. Reduction of Contaminant Mobility at the TNX OD Outfall Delta Through the use of Apatite and Zero-Valent Iron as Sediment Amendments (U). WSRC-TR-2002-00370, Rev. 0 Westinghouse Savannah River Company, Aiken, SC.

Knox, A.S., D.I. Kaplan, D.C. Adriano, T.G. Hinton, and M.D. Wilson. 2003. Apatite and Phillipsite as Sequestering Agents for Metals and Radionuclides. J. Environ. Qual. 32: 515-525.

Knudsen, A. C., and M. E. Gunter. 2002. Sedimentary Phosphorites-An Example: Phophoria Formation, Southeastern Idaho, USA. In: M.J. Kohn, J. Rakovan and J.M. Hughes (Eds), Phosphates - Geochemical, Geobiological, and Materials Importance, Reviews in mineralogy and geochemistry, Vol. 48 Mineralogical Society of America, Washington, DC.

Kuo, S. 1996. Phosphorus. In: D. L. Sparks (ed), Methods of Sediment Analysis, Part 3 Chemical Methods. pp. 869 - 919. Sediment Science Society of America, Madison, WI. Ma, Q.Y., T.J. Logan, and S.J. Traina. 1995. Lead immobilization from aqueous solutions and contaminated sediments using phosphate rocks. Environ. Sci. Technol. 29: 1118-1126.

Melita, N. M. Conklin, and J. Ferrell. Electrochemical Study of Arsenate and Water Reduction on Iron Media Used for Arsenic Removal from Potable Water. Environ. Sci. Technol. 36(14):3188-3193.

Miller, W. P. and D. M. Miller. 1987. "A Micro-Pipette Method for Sediment Mechanical Analysis." Communications In Sediment Sci., Plant Anal. 18(1) 1-15.

Runchal, A. 2000. PORFLOW: A Model for Fluid Flow, Heat, and Mass Transport in Multifluid, Multiphase, Fractured, or Porous Media. User's manual - Version 4.0. Analytical and Computational Research, Inc (ACRI), Bel Air, CA.

Ryan, J.A., P. Zhang, D. Hesterberg, L. Chou, and D.E. Sayers. 2001. Formation of chloropyromorphite in a lead-contaminated sediment amended with hydroxyapatite. Environ. Sci. Technol. 35:3798-3803.

Sass, B. M., and D. Rai. 1987. Solubility of Amorphous Chromium(III)-Iron(III) Hydroxide Solid Solutions. Inorganic Chemistry. 26:2228-2232. 
WSRC-TR-2003-00579, REV. 0

Seaman, J.C., T. Meehan, and P.M. Bertsch. 2001. Immobilization of cesium-137 and uranium in contaminated sediments using sediment amendments. J. Environ. Qual. 30:1206-1213.

Sparks, D.O. 1996. Methods of Sediment Analysis, Part 3 - Chemical Methods. Sediment Science Society of America, Inc., Madison, Wisconsin.

Suzuki, T., T. Hatsushika, and M. Miyake. 1982. Synthetic Hydroxyapatite as inorganic cations exchangers. J. Chem. Soc., Faraday Trans. I. 78:3605-3611.

Suzuki, T., T. Hatsushika, and Y. Hayakawa. 1981. Synthetic Hydroxyapatite employed as inorganic cation-exchangers. J. Chem. Soc., Faraday Trans. I. 77:1059-1062.

Thomas, G. W. 1996. Sediment pH and Sediment Acidity. In: D. H. Sparks (ed.) Methods of Sediment Analysis, Part 3, Chemical Methods, Sediment Science Society of America and American Society of Agronomy, Madison, Wisconsin.

U.S. EPA. 1992. Toxicity Characteristic Leaching procedure. Method 1311, Rev.0. In SW-846: Test methods for evaluating solid waste, physical/chemical methods. Office of Solid Waste, Washington, DC.

U.S. EPA, 1999. Identification and Listing of Hazardous Waste, Toxicity Characteristics. 40 CFR 261.24, Federal Register, vol. 18, no. 261, pp. 55-56, July 1, 1999.

WSRC (Westinghouse Savannah River Company). 1990. RFI/RI with BRA for the TNX OD Outfall Delta, Lower Discharge Gully and swamp operable Unit. WSRC_RP-98-4158, Rev. 0. Westinghouse Savannah River Company, Aiken, SC.

Winer, B. J. 1971. Statistical Principles in Experimental Design. McGraw-Hill, Inc.

Wright, J. 1990. Condont Apatite: Structure and Geochemistry. In: J. Carter (Ed.), Metazoan Biomineralization:Patterns, Processes and Evalutionary Trends. Pp. 445-459, Van Nostrand Reinhold, New York.

Wright, J. V., L. M. Peurrung, T. E. Moody, J. L. Conca, X. Chen, P. P. Didzerekis, and E. Wyse. 1995. In Situ Immobilization of Heavy Metals: Apatite Mineral Formations. Technical Report to the Strategic Environmental Research and Development Program, Department of Defense, Pacific Northwest National Laboratory, Richland, WA p. 154. 
WSRC-TR-2003-00579, REV. 0

Appendix A: Additional Data from the Laboratory Studies 
Table 1A. Sorption of Fe on the TNX OD sediment

\begin{tabular}{|c|c|c|c|c|c|c|c|c|c|c|c|c|}
\hline & $\begin{array}{l}\text { Treatment } \\
\text { description }\end{array}$ & Rep. & $\begin{array}{c}\begin{array}{c}\text { Initial } \\
\text { conc. } \\
\text { of } \mathrm{Fe}^{\mathrm{a}} \\
{[\mathrm{mg} / \mathrm{kg}]}\end{array} \\
\end{array}$ & \begin{tabular}{|c|} 
Initial \\
$\mathrm{pH}$
\end{tabular} & $\begin{array}{c}\text { Initial } \\
\mathrm{Eh} \\
{[\mathrm{mV}]}\end{array}$ & \begin{tabular}{|c}
$\mathrm{pH}$ \\
after \\
4 days
\end{tabular} & \begin{tabular}{|c|} 
Eh \\
after \\
4 days \\
{$[\mathrm{mV}]$} \\
\end{tabular} & $\begin{array}{c}\text { Absorbance } \\
{[\mathrm{mm}]}\end{array}$ & $\begin{array}{l}\text { Final Fe } \\
\text { conc. }^{\circ} \\
{[\mathrm{mg} / \mathrm{kg}]}\end{array}$ & \begin{tabular}{|c|} 
Corrected \\
Final Fe \\
conc. $^{\mathrm{c}}$ \\
{$[\mathrm{mg} / \mathrm{kg}]$}
\end{tabular} & $\begin{array}{c}\text { Kd for } \\
\text { total Fe } \\
{[\mathrm{g} / \mathrm{L}]}\end{array}$ & $\begin{array}{c}\text { Fe } \\
\text { sorbed } \\
{[\mu \mathrm{g} / \mathrm{g}]}\end{array}$ \\
\hline $0-1$ & No sediment & 1 & 0.0 & 6.91 & 205 & 6.95 & 188.4 & 0.02 & -0.219 & & & \\
\hline $0-1$ & No sediment & 2 & 0.0 & 7.03 & 204.5 & 7.13 & 191.6 & 0.02 & -0.219 & & & \\
\hline $0-2$ & No sediment & 3 & 0.0 & 7.13 & 200.6 & 7.1 & 196.7 & 0.02 & -0.219 & & & \\
\hline $1-1$ & sediment & 1 & 0.0 & 6.69 & 203.3 & 6.25 & 217.5 & 0.0743 & 0.297 & & & \\
\hline $1-2$ & sediment & 2 & 0.0 & 6.65 & 204.4 & 6.36 & 219.5 & 0.0978 & 0.348 & & & \\
\hline $1-3$ & sediment & 3 & 0.0 & 6.75 & 204.3 & 6.33 & 223 & 0.0882 & 0.352 & & & \\
\hline $2-1$ & sediment & 1 & 0.9 & 6.62 & 106.8 & 6.23 & 234.9 & 0.1067 & 0.445 & 0.113 & 141 & 18 \\
\hline $2-2$ & sediment & 2 & 0.9 & 6.5 & 93.5 & 6.31 & 234 & 0.1087 & 0.458 & 0.126 & 124 & 18 \\
\hline $2-3$ & sediment & 3 & 0.9 & 6.49 & 107 & 6.17 & 240.5 & 0.106 & 0.440 & 0.108 & 148 & 18 \\
\hline 3-1 & sediment & 1 & 2.3 & 6.52 & 93 & 6.09 & 244.9 & 0.1024 & 0.451 & 0.119 & 363 & 45 \\
\hline $3-2$ & sediment & 2 & 2.3 & 6.49 & 86.3 & 6.13 & 249.5 & 0.1026 & 0.512 & 0.180 & 232 & 44 \\
\hline $3-3$ & sediment & 3 & 2.3 & 6.53 & 89.9 & 6.18 & 248.2 & 0.1012 & 0.443 & 0.111 & 393 & 46 \\
\hline \begin{tabular}{|l|}
$4-1$ \\
\end{tabular} & sediment & 1 & 4.6 & 6.38 & 86.7 & 6.08 & 268 & 0.1271 & 0.577 & 0.245 & 353 & 89 \\
\hline $4-2$ & sediment & 2 & 4.6 & 6.41 & 86.1 & 6.04 & 271.6 & 0.1143 & 0.494 & 0.162 & 546 & 91 \\
\hline $4-3$ & sediment & 3 & 4.6 & 6.41 & 85.7 & 6.01 & 267.1 & 0.1232 & 0.552 & 0.220 & 396 & 89 \\
\hline $5-1$ & sediment & 1 & 13.8 & 6.11 & 85.1 & 5.71 & 266.9 & 0.1053 & 0.534 & 0.202 & 1343 & 273 \\
\hline \begin{tabular}{|l|}
$5-2$ \\
\end{tabular} & sediment & 2 & 13.8 & 6.1 & 87.2 & 5.74 & 242.3 & 0.1453 & 0.624 & 0.292 & 919 & 270 \\
\hline $5-3$ & sediment & 3 & 13.8 & 6.15 & 84.2 & 5.71 & 259.3 & 0.103 & 0.515 & 0.183 & 1481 & 274 \\
\hline \begin{tabular}{|l|}
$6-1$ \\
\end{tabular} & sediment & 1 & 23.0 & 5.93 & 92.5 & 5.32 & 215.3 & 0.1238 & 0.601 & 0.269 & 1683 & 455 \\
\hline $6-2$ & sediment & 2 & 23.0 & 6.02 & 84.4 & 5.34 & 211.6 & 0.1139 & 0.532 & 0.200 & 2280 & 457 \\
\hline $6-3$ & sediment & 3 & 23.0 & 5.97 & 91.5 & 5.35 & 202 & 0.1139 & 0.532 & 0.200 & 2273 & 456 \\
\hline
\end{tabular}

a Initial concentration of $\mathrm{Fe}$ in each treatment was based on the spike solution Fe concentration $(368 \mathrm{mg} / \mathrm{kg})$

$\mathrm{b}$ The final Fe concentration was calculated from the calibration equation (Figure 1A)

c The final Fe concentration was corrected for a background concentration of Fe from the sediment B5 from the TNX OD and from the TNX OD water 
Table 2A. Sorption of P on the TNX OD sediment

\begin{tabular}{|c|c|c|c|c|c|c|c|c|}
\hline \begin{tabular}{|l|} 
Treat \\
ment
\end{tabular} & $\begin{array}{l}\text { Treatment } \\
\text { description }\end{array}$ & \begin{tabular}{|c|} 
Initial \\
con. \\
of added \\
$\mathrm{P}^{\mathrm{a}}$ \\
{$[\mathrm{mg} / \mathrm{kg}]$} \\
\end{tabular} & $\begin{array}{c}\text { Initial } \\
\mathrm{pH}\end{array}$ & Final $\mathrm{pH}$ & Absorbance & $\begin{array}{c}\text { Final } \mathrm{P} \\
\text { conc. }^{\mathrm{b}} \\
{[\mathrm{mg} / \mathrm{kg}]}\end{array}$ & $\begin{array}{c}\text { Kd for } P \\
{[\mathrm{~g} / \mathrm{L}]}\end{array}$ & \begin{tabular}{|c}
$\mathrm{P}$ \\
sorbed \\
{$[\mathrm{mg} / \mathrm{kg}]$}
\end{tabular} \\
\hline I & No sediment & & 6.98 & 7.03 & 0.0137 & 0.073 & & \\
\hline I & No sediment & & 6.99 & 7.06 & 0.0459 & 0.466 & & \\
\hline I & No sediment & & 7.02 & 7.08 & 0.0086 & 0.011 & & \\
\hline II & Sediment & 0.95 & 6.73 & 6.63 & 0.0625 & 0.852 & 1.9 & 1.6 \\
\hline II & Sediment & 0.95 & 6.69 & 6.3 & 0.0174 & 0.302 & 35.5 & 10.7 \\
\hline II & Sediment & 0.95 & 6.67 & 6.28 & 0.0303 & 0.459 & 17.7 & 8.1 \\
\hline III & Sediment & 1.72 & 6.68 & 6.3 & 0.0847 & 1.122 & 8.7 & 9.8 \\
\hline III & Sediment & 1.72 & 6.64 & 6.3 & 0.0111 & 0.225 & 109.6 & 24.6 \\
\hline III & Sediment & 1.72 & 6.59 & 6.31 & 0.0129 & 0.247 & 98.4 & 24.3 \\
\hline IV & Sediment & 4.02 & 6.59 & 6.28 & 0.0585 & 0.803 & 65.9 & 52.9 \\
\hline IV & Sediment & 4.02 & 6.6 & 6.32 & 0.012 & 0.236 & 263.9 & 62.2 \\
\hline IV & Sediment & 4.02 & 6.65 & 6.31 & 0.012 & 0.236 & 264.7 & 62.4 \\
\hline $\mathrm{V}$ & Sediment & 7.85 & 6.56 & 6.37 & 0.0228 & 0.367 & 336.0 & 123.5 \\
\hline $\mathrm{V}$ & Sediment & 7.85 & 6.57 & 6.35 & 0.0807 & 1.074 & 104.1 & 111.8 \\
\hline $\mathrm{V}$ & Sediment & 7.85 & 6.63 & 6.3 & 0.0294 & 0.448 & 272.1 & 121.9 \\
\hline VI & Sediment & 19.35 & 6.54 & 6.47 & 0.0649 & 0.881 & 345.6 & 304.4 \\
\hline VI & Sediment & 19.35 & 6.55 & 6.45 & 0.0741 & 0.993 & 304.2 & 302.1 \\
\hline VI & Sediment & 19.35 & 6.57 & 6.36 & 0.0871 & 1.152 & 260.2 & 299.7 \\
\hline VII & Sediment & 38.51 & 6.55 & 6.34 & 0.2735 & 3.425 & 169.0 & 578.7 \\
\hline VII & Sediment & 38.51 & 6.51 & 6.37 & 0.3317 & 4.135 & 137.5 & 568.4 \\
\hline VII & Sediment & 38.51 & 6.43 & 6.37 & 0.2391 & 3.005 & 194.8 & 585.3 \\
\hline VIII & Sediment & 76.84 & 6.41 & 6.37 & 0.8699 & 10.698 & 101.9 & 1090.4 \\
\hline VIII & Sediment & 76.84 & 6.38 & 6.35 & 0.8956 & 11.011 & 98.5 & 1084.7 \\
\hline VIII & Sediment & 76.84 & 6.35 & 6.32 & 0.8644 & 10.631 & 102.9 & 1094.2 \\
\hline
\end{tabular}

a Initial concentration of $\mathrm{P}$ in each treatment was based on the $\mathrm{P}$ spike solution concentration $(507 \mathrm{mg} / \mathrm{kg})$

$\mathrm{b}$ The final $\mathrm{P}$ concentration was calculated from the calibration equation (Figure 2) 
Figure 1A. The calibration equation for Fe absorbance

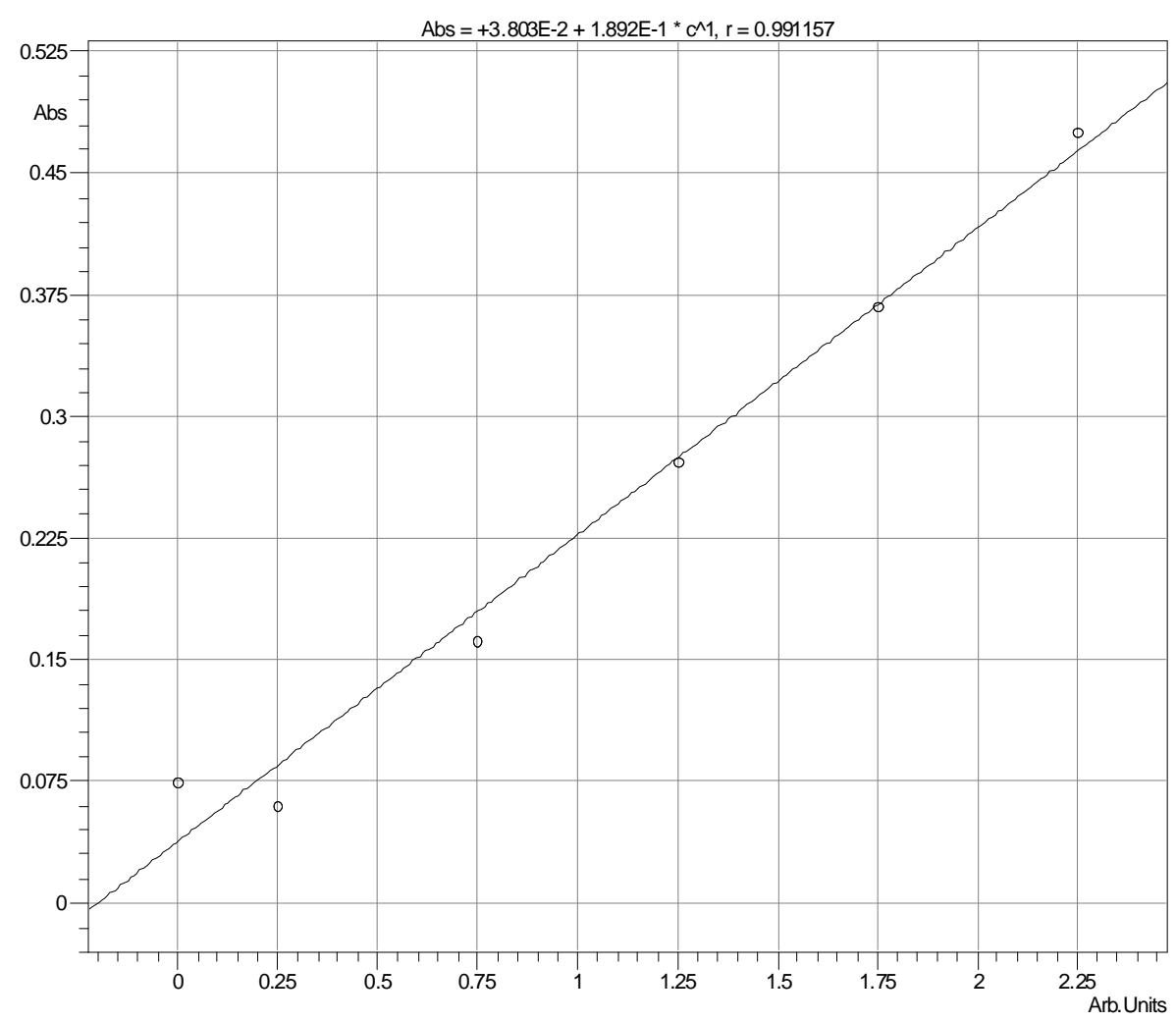

Concentration of $\mathrm{Fe}$ (total) in $\mathrm{mg} / \mathrm{kg}$.

No. Name Conc. Fact Value

or [Abs]

$\begin{array}{llccr} & & \mathrm{mg} / \mathrm{kg} & & \\ 1 & 1-1 \mathrm{~A} & 0 & 1 & 0.0743 \\ 2 & 250 \mathrm{MG} / \mathrm{KG} & 0.25 & 1 & 0.0598 \\ 3 & 750 \mathrm{MG} / \mathrm{KG} & 0.75 & 1 & 0.1615 \\ 4 & 1250 \mathrm{MG} / \mathrm{K} & 1.25 & 1 & 0.2721 \\ & \mathrm{G} & & & \\ 5 & 1750 \mathrm{MG} / \mathrm{K} & 1.75 & 1 & 0.3679 \\ & \mathrm{G} & & & \\ 6 & 2250 \mathrm{PPB} & 2.25 & 1 & 0.475\end{array}$


Figure 2 A. The calibration equation for $\mathrm{P}$ absorbance

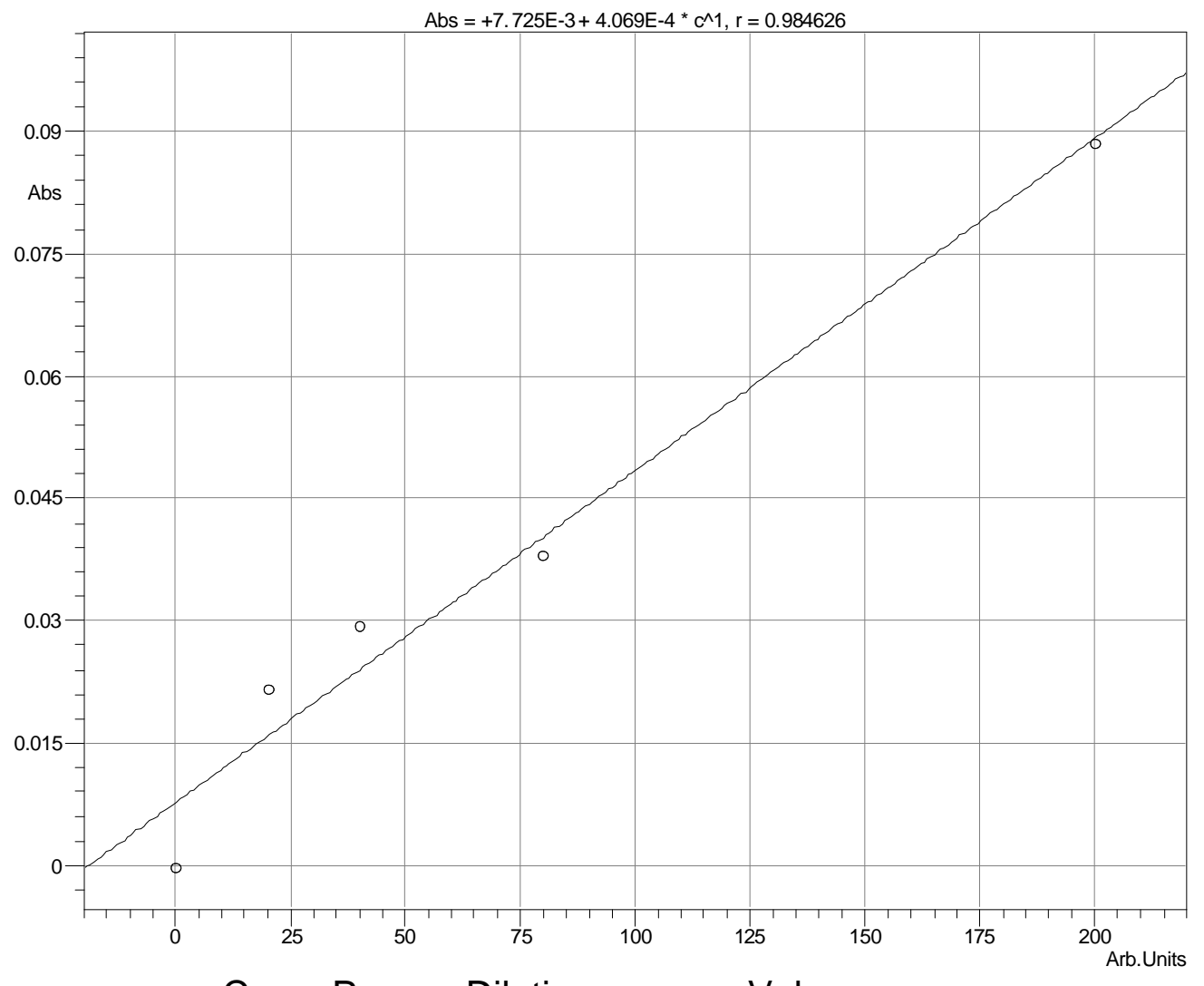

$\begin{array}{lrrr}\text { Standard } & \begin{array}{r}\text { Conc. P } \\ (\mathrm{ppb})\end{array} & \begin{array}{r}\text { Dilution } \\ \text { Factor }\end{array} & \begin{array}{r}\text { Value } \\ \text { (Arb.Units) }\end{array} \\ \text { BLANK } & 0 & 1 & -0.0003 \\ 20 \mathrm{PPB} & 20 & 1 & 0.0217 \\ 40 \mathrm{PPB} & 40 & 1 & 0.0293 \\ \text { 80 PPB } & 80 & 1 & 0.0378 \\ 120 \mathrm{PPB} & 120 & 1 & 0.0817 \\ 200 \text { PPB } & 200 & 1 & 0.0884\end{array}$


WSRC-TR-2003-00579, REV. 0

\section{Appendix B: Work Instructions for the Laboratory Studies}




\section{Phosphorous Sorption Isotherm}

Objective: The objective of the phosphate sorption isotherm experiments was to measure the phosphate distribution coefficient, $\mathrm{K}_{\mathrm{d}}$, in the TNX OD sediment (from coordinate B5) to permit calculation of the effective diffusion coefficient, $D_{\text {eff, was used the Fick's }}$ second law describing one-dimensional diffusion of solutes in soil as (Dragun, 1998):

$$
\begin{aligned}
& \delta \mathrm{c} / \delta \mathrm{t}=\left(\mathrm{D}_{\mathrm{eff}} / \mathrm{R}_{\mathrm{F}}\right)\left(\delta^{2} \mathrm{c} / \delta \mathrm{x}^{2}\right) \\
& \mathrm{c} \quad=\text { concentration of a solute }\left(\mathrm{mg} / \mathrm{cm}^{3}\right) \\
& \mathrm{t} \quad=\text { time (s) } \\
& D_{\text {eff }}=\text { measured diffusion coefficient }\left(\mathrm{cm}^{2} / \mathrm{s}\right) \text {, } \\
& \mathrm{R}_{\mathrm{F}}=\text { retardation factor (calculated from Equation 2) } \\
& \mathrm{X}=\text { direction of diffusion }(\mathrm{cm}) \\
& \mathrm{R}_{\mathrm{F}}=1+\left(\rho_{\mathrm{b}} / \phi_{\mathrm{e}}\right) \mathrm{K}_{\mathrm{d}} \\
& \left.\rho_{b}=\text { the porous media bulk density (mass/length }{ }^{3}\right)(\text { taken from literature for a } \\
& \text { sediment with the texture of sediment B5, loamy sand, } 1.55 \mathrm{~g} / \mathrm{cm}^{3} \text { ) (Dragun, } \\
& \text { 1998) } \\
& \phi_{\mathrm{e}}=\text { the effective porosity at saturation of media (taken from literature for a } \\
& \text { sediment with the texture of sediment B5, loamy sand, 0.47) (Hillel, 1980) } \\
& \mathrm{D}_{\mathrm{e}}=\mathrm{D}_{\mathrm{o}} / \mathrm{R}_{\mathrm{F}} \\
& \mathrm{D}_{\mathrm{e}}=\text { measured diffusion coefficient, } \\
& \mathrm{D}_{\mathrm{o}}=\text { the aqueous diffusion coefficient (taken from literature), } \\
& \mathrm{R}_{\mathrm{F}}=\text { retardation factor (calculated from Equation 2) }
\end{aligned}
$$

\section{Solutions:}

1. $0.01 M \mathrm{NaCl}=0.52 \mathrm{~g} / \mathrm{L}$

2. $400 \mathrm{mg} / \mathrm{kg}$ Stock P Solution $=0.585 \mathrm{~g} / \mathrm{L} \mathrm{NaH} \mathrm{PO}_{4}-\mathrm{H}_{2} \mathrm{O}$

\section{Method:}

1. Label 50-ml centrifuge tubes as shown in Table 1.

2. Add 2-g of well mixed B-5 sediment to each tube. Record tube tare weight and actual sediment weight.

3. Pre-equilibrate sediment with $23-\mathrm{hr}$ washes followed by a 1 overnight $40-\mathrm{mL}$ wash with $0.01 M \mathrm{NaCl}$ solution. Centrifuge between wash steps. Be sure that no sediment is loss between centrifuge steps, i.e., error on the side of leaving too much liquid in tube rather then losing any solids. Record final wet weight.

4. Add appropriate volumes of $\mathrm{NaCl}$ and Stock P Solution to tubes as shown in Table 1.

5. Measure initial $\mathrm{pH}$.

6. Leave samples on platform shaker for 4 days.

7. Measure final $\mathrm{pH}$.

8. Centrifuge. Then measure $\mathrm{P}$ concentration of aqueous phase using the Murphy-Riley method as modified by Kuo (1996). ${ }^{1}$

9. Calculate $K_{d}$ or Freundlich terms, whichever is appropriate.

\footnotetext{
${ }^{1}$ Kuo, S. 1996. Phosphorus. In: D. Sparks (ed.) Methods of Sediment Analysis, Part 3 - Chemical Methods. p 859-921. Sediment Science of America, Inc., Madison, WI.
} 
Table 1B. Sample identification.

\begin{tabular}{|c|c|c|c|c|c|c|c|c|c|}
\hline $\begin{array}{c}\text { Tube } \\
\text { ID\# }\end{array}$ & $\begin{array}{c}\text { Preat- } \\
\text { ment } \\
(\mathrm{mg} / \mathrm{L})\end{array}$ & Rep. & $\begin{array}{c}\text { Tube } \\
\text { Tare } \\
\text { Wt. }(\mathrm{g})\end{array}$ & $\begin{array}{c}\text { Sediment } \\
\text { Dry Wt. } \\
(\mathrm{g})\end{array}$ & $\begin{array}{c}\text { Sediment } \\
\text { Wet Wt. } \\
(\mathrm{g})\end{array}$ & $\begin{array}{c}0.013 \\
M \mathrm{NaCl} \\
\text { Added } \\
(\mathrm{mL})\end{array}$ & $\begin{array}{c}400- \\
\mathrm{mg} / \mathrm{kg} \\
\text { Stock } \\
\text { PSoln. } \\
\text { Added } \\
(\mathrm{mL})\end{array}$ & $\begin{array}{c}\text { Initial } \\
\mathrm{pH}\end{array}$ & $\begin{array}{c}\text { Final } \\
\mathrm{pH}\end{array}$ \\
\hline $0-1$ & 0 & 1 & & & & 40 & 0 & & \\
\hline $0-2$ & 0 & 2 & & & & 40 & 0 & & \\
\hline $0-3$ & 0 & 3 & & & & 40 & 0 & & \\
\hline $1-1$ & 1 & 1 & & & & 39.9 & 0.1 & & \\
\hline $1-2$ & 1 & 2 & & & & 39.9 & 0.1 & & \\
\hline $1-3$ & 1 & 3 & & & & 39.9 & 0.1 & & \\
\hline $2.5-1$ & 2.5 & 1 & & & & 39.75 & 0.25 & & \\
\hline $2.5-2$ & 2.5 & 2 & & & & 39.75 & 0.25 & & \\
\hline $2.5-3$ & 2.5 & 3 & & & & 39.75 & 0.25 & & \\
\hline $5-1$ & 5 & 1 & & & & 39.5 & 0.5 & & \\
\hline $5-2$ & 5 & 2 & & & & 39.5 & 0.5 & & \\
\hline $5-3$ & 5 & 3 & & & & 39.5 & 0.5 & & \\
\hline $12.5-1$ & 12.5 & 1 & & & & 38.75 & 1.25 & & \\
\hline $12.5-2$ & 12.5 & 2 & & & & 38.75 & 1.25 & & \\
\hline $12.5-3$ & 12.5 & 3 & & & & 38.75 & 1.25 & & \\
\hline $25-1$ & 25 & 1 & & & & 37.5 & 2.5 & & \\
\hline $25-2$ & 25 & 2 & & & & 37.5 & 2.5 & & \\
\hline $25-3$ & 25 & 3 & & & & 37.5 & 2.5 & & \\
\hline $50-1$ & 50 & 1 & & & & 35 & 5 & & \\
\hline $50-2$ & 50 & 2 & & & & 35 & 5 & & \\
\hline $50-3$ & 50 & 3 & & & & 35 & 5 & & \\
\hline
\end{tabular}


WSRC-TR-2003-00579, REV. 0

\section{Ferrous Iron Sorption Isotherm}

\section{Solutions:}

3. $400 \mathrm{mg} / \mathrm{kg} \mathrm{Stock} \mathrm{FeCl}_{2} \times 4 \mathrm{H}_{2} \mathrm{O}$ Solution $=0.7117 \mathrm{~g}$ of $\mathrm{FeCl}_{2} \times 4 \mathrm{H}_{2} \mathrm{O}$ in $500 \mathrm{~mL} \mathrm{H}_{2} \mathrm{O}$ Calculated as followed:

$\mathrm{FeCl}_{2} \times 4 \mathrm{H}_{2} \mathrm{O} \mathrm{FW}=198.746 \mathrm{~g} / \mathrm{mol}$

Ratio of $\mathrm{Fe} / \mathrm{FeCl}_{2} \times 4 \mathrm{H}_{2} \mathrm{O}=1 / 0.281=3.559$

We need $200 \mathrm{mg}$ of $\mathrm{Fe}^{2+}$ in $500 \mathrm{ml}$ of water to make $400 \mathrm{mg} / \mathrm{kg} \mathrm{Fe}{ }_{2+}$ solution:

$200 \mathrm{mg} \times 3.559=711.7 \mathrm{mg} \mathrm{FeCl}{ }_{2} \times \mathrm{H}_{2} \mathrm{O}$

\section{Method:}

10. Label 50-ml centrifuge tubes as shown in Table 1.

11. Add 2-g of well mixed B5 sediment to each tube. Record tube tare weight and actual sediment weight.

12. Pre-equilibrate sediment with 23 -hr washes followed by a 1 overnight $40-\mathrm{mL}$ wash with the TNX OD swamp water. Centrifuge between wash steps. Be sure that no sediment is loss between centrifuge steps, i.e., error on the side of leaving too much liquid in tube rather then losing any solids. Record final wet weight.

13. Add appropriate volumes of swamp water from the TNX OD and Stock $\mathrm{FeCl}_{2}$ Solution to tubes as shown in Table 1.

14. Measure initial $\mathrm{pH}$ and Eh.

15. Leave samples on platform shaker for 4 days.

16. Measure final $\mathrm{pH}$ and $\mathrm{Eh}$.

17. Centrifuge. Then measure $\mathrm{Fe}$ total and $\mathrm{Fe}^{2+}$ concentration of aqueous phase by calorimetric determination of ferrous iron and ferric iron by 1,10-phenanthroline method (page 659).

18. Calculate $\mathrm{K}_{\mathrm{d}}$ or Freundlich terms, whichever is appropriate.

\section{1,10 Phenanthroline method (or EPA methods: Fe ${ }^{2+}$ : EPA\#FM3500 and Total Fe: EPA\#200.7)}

\section{Special Apparatus:}

Visible or ultraviolet (UV)/visible spectrophotometer

\section{Reagents:}

1. Ammonium acetate $\left(\mathrm{NH}_{4} \mathrm{C}_{2} \mathrm{H}_{3} \mathrm{O}_{2}\right), 5 M$.

2. Hydroxylamine hydrochloride $\left(\mathrm{NH}_{2} \mathrm{OH}{ }^{\bullet} \mathrm{HCl}\right), 10 \%\left(10 \mathrm{~g} \mathrm{NH}_{2} \mathrm{OH}{ }^{\cdot} \mathrm{HCl}\right.$ dilute to $100 \mathrm{~mL}$ total volume with deionized water).

3. 1,10-phenanthroline reagent. Dissolve $0.30 \mathrm{~g}$ of 1,10-phenanthroline monohydrate in water by heating the mixture to $80^{\circ} \mathrm{C}$. Cool the solution and add water to a final volume of $100 \mathrm{~mL}$.

4. Hydrochloric acid, $6 M$.

5. Standard Fe solution, $100 \mathrm{mg} \mathrm{Fe} \mathrm{L}^{-1}$.

6. Standard Fe solutions, $5 \mathrm{mgL}^{-1}$. Add $10 \mathrm{~mL}$ of $18 \mathrm{M} \mathrm{H}_{2} \mathrm{SO}_{4}$ to $50.0 \mathrm{~mL}$ of standard $\mathrm{Fe}$ solution having $100 \mathrm{mg} \mathrm{L}^{-1}$ of $\mathrm{Fe}$, and dilute the solution to $1 \mathrm{~L}$ with deionized water. 
Table 2B. Sample identification, Ferrous Fe sorption Isotherm exp.

\begin{tabular}{|c|c|c|c|c|c|c|c|c|}
\hline $\begin{array}{c}\text { Treat } \\
\text { ment } \\
\text { ID\# }\end{array}$ & $\begin{array}{c}\text { Tube } \\
\text { ID }\end{array}$ & $\begin{array}{c}\mathrm{Fe}^{2+} \\
\text { Treat- } \\
\text { ment } \\
(\mathrm{mg} / \\
\mathrm{L})\end{array}$ & Rep. & & $\begin{array}{c}\text { Tube } \\
\text { Tare Wt. } \\
(\mathrm{g})\end{array}$ & $\begin{array}{c}\text { Sediment } \\
\text { Dry Wt. } \\
(\mathrm{g})\end{array}$ & $\begin{array}{c}\text { TNX } \\
\text { swamp } \\
\text { water } \\
\text { Added } \\
(\mathrm{mL})\end{array}$ & $\begin{array}{c}400- \\
\mathrm{mg} / \mathrm{kg} \\
\text { Stock } \\
\mathrm{Fe}^{2+} \\
\text { Soln. } \\
\text { Added } \\
(\mathrm{mL})\end{array}$ \\
\hline 0 & $0-1$ & 0 & 1 & $\begin{array}{c}\text { No } \\
\text { sediment }\end{array}$ & 11.6467 & - & 40 & 0 \\
\hline 0 & $0-1$ & 0 & 2 & $\begin{array}{c}\text { No } \\
\text { sediment }\end{array}$ & 11.6842 & - & 40 & 0 \\
\hline 0 & $0-2$ & 0 & 3 & $\begin{array}{c}\text { No } \\
\text { sediment }\end{array}$ & 11.7733 & - & 40 & 0 \\
\hline 1 & $1-1$ & 0 & 1 & Sediment & 11.7092 & 2.0088 & 40 & 0 \\
\hline 1 & $1-2$ & 0 & 2 & Sediment & 11.7147 & 2.0073 & 40 & 0 \\
\hline 1 & $1-3$ & 0 & 3 & Sediment & 11.6449 & 2.0074 & 40 & 0 \\
\hline 2 & $2-1$ & 1 & 1 & Sediment & 11.6970 & 2.0042 & 39.900 & 0.1 \\
\hline 2 & $2-2$ & 1 & 2 & Sediment & 11.7663 & 2.0022 & 39.900 & 0.1 \\
\hline 2 & $2-3$ & 1 & 3 & Sediment & 11.6417 & 2.0037 & 39.900 & 0.1 \\
\hline 3 & $3-1$ & 2.5 & 1 & Sediment & 11.6541 & 2.0092 & 39.75 & 0.25 \\
\hline 3 & $3-2$ & 2.5 & 2 & Sediment & 11.7088 & 2.0070 & 39.75 & 0.25 \\
\hline 3 & $3-3$ & 2.5 & 3 & Sediment & 11.6413 & 2.0059 & 39.75 & 0.25 \\
\hline 4 & $4-1$ & 5 & 1 & Sediment & 11.7677 & 2.0013 & 39.5 & 0.5 \\
\hline 4 & $4-2$ & 5 & 2 & Sediment & 11.7413 & 2.0001 & 39.5 & 0.5 \\
\hline 4 & $4-3$ & 5 & 3 & Sediment & 11.6786 & 2.0043 & 39.5 & 0.5 \\
\hline 5 & $5-1$ & 15 & 1 & Sediment & 11.6519 & 2.0061 & 38.5 & 1.5 \\
\hline 5 & $5-2$ & 15 & 2 & Sediment & 11.7342 & 2.0096 & 38.5 & 1.5 \\
\hline 5 & $5-3$ & 15 & 3 & Sediment & 11.6850 & 2.0032 & 38.5 & 1.5 \\
\hline 6 & $6-1$ & 25 & 1 & Sediment & 11.6630 & 2.0071 & 37.5 & 2.5 \\
\hline 6 & $6-2$ & 25 & 2 & Sediment & 11.7428 & 2.0031 & 37.5 & 2.5 \\
\hline 6 & $6-3$ & 25 & 3 & Sediment & 11.7442 & 2.0093 & 37.5 & 2.5 \\
\hline & & & & & & & & \\
\hline
\end{tabular}




\section{Solubility test of collected apatite samples}

March 10, 2003

\section{Method:}

1. Label 50-ml centrifuge tubes as shown in Table 1.

2. Add 1-g of well mixed apatite sample to tube following the experimental design (Table 1). Record tube tare weight and actual weight of each apatite sample.

3. Add $10 \mathrm{ml}$ of DI water to each tube.

4. Measure initial $\mathrm{pH}$. If needed adjust $\mathrm{pH}$ to $~ 7.5$.

5. Leave sample on platform shaker for 12 days.

6. Measure final $\mathrm{pH}$.

7. Centrifuge. Then measure $\mathrm{P}$ concentration of aqueous phase using the Marphy-Riley method as modified by Kuo (1993).

8. Sub-sample of aqueous phase will be analyzed for 30 elements by ICP-AES. 
Table 3B. Solubility test of the collected phosphate rocks from different locations.

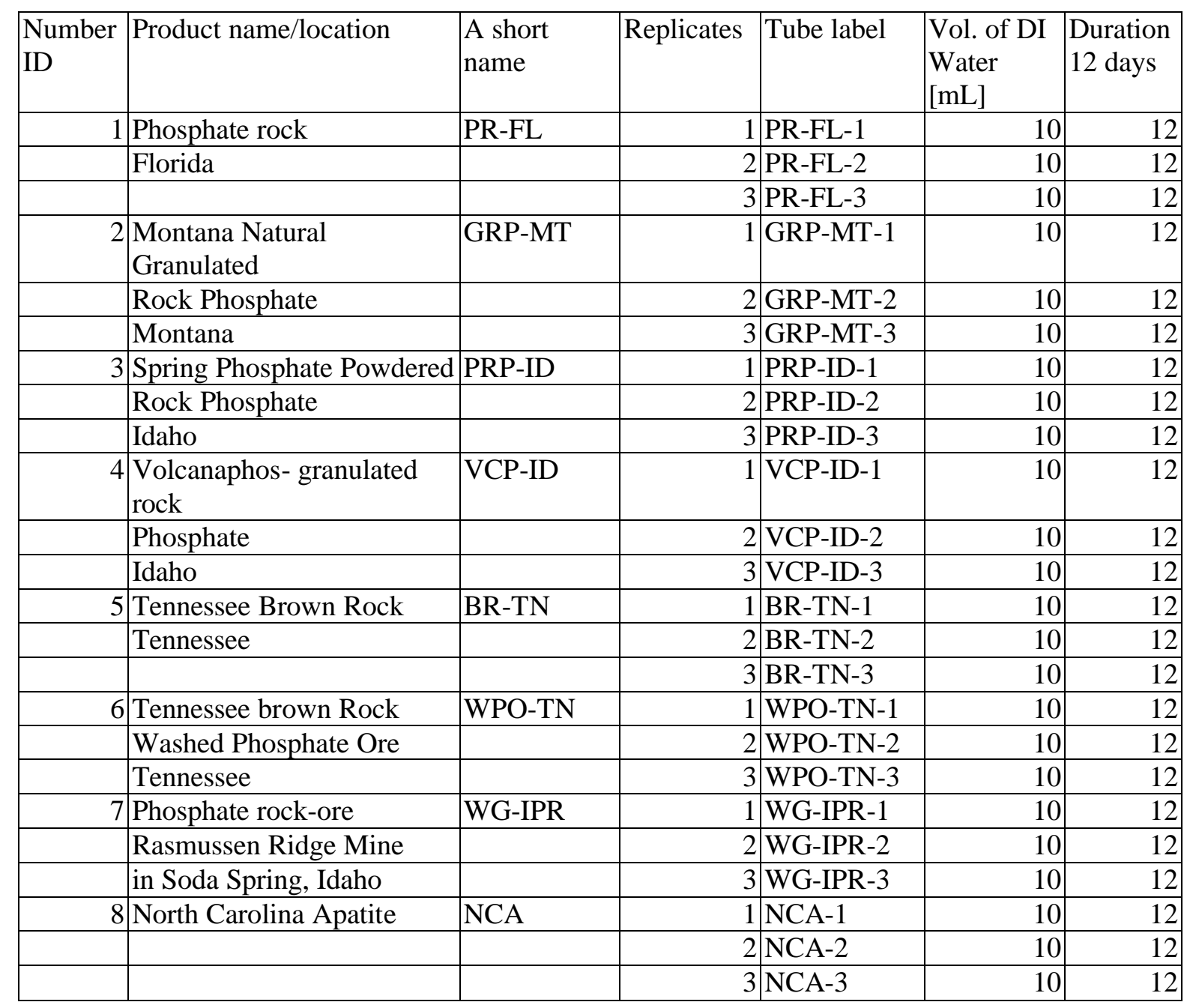


WSRC-TR-2003-00579, REV. 0

\section{A micro-pipette method $^{2}$ of apatite samples from different sources}

\section{Reagents:}

$5 \%\left(\mathrm{NaPO}_{3}\right)_{13}-50 \mathrm{ml}$ (needed only $10 \mathrm{ml}$ )

$2.5 \mathrm{~g}$ of $\%\left(\mathrm{NaPO}_{3}\right)_{13}$ and dilute to $50 \mathrm{ml}$ with DI water

$\mathrm{M} \mathrm{naOH}-50 \mathrm{ml}$ (needed only $10 \mathrm{ml}$ )

$2 \mathrm{~g}$ of naOH and dilute to $50 \mathrm{ml}$ with DI water

Dispersant Solution

$10 \mathrm{ml}$ of $5 \% \%\left(\mathrm{NaPO}_{3}\right)_{13}$ and $10 \mathrm{ml}$ of $1.0 \mathrm{M} \mathrm{NaOH}$ in $1 \mathrm{~L}$ of DI water

\section{Samples/Method}

$4 \mathrm{~g}$ sample weights were used with $40 \mathrm{ml}$ of dispersant solution. Samples were placed on the shaker platform and shaking overnight at $55 \mathrm{rev} / \mathrm{min}$.

After dispersing, the samples were removed from the shaker and caps removed after a final shaking. After a settling time of clay fraction ( $1 \mathrm{hr}$ and 50 minutes) the pipette tip $(5 \mathrm{~mL})$ was lowered into the suspension with the hand supported by an appropriately sized block of wood and the sample withdrawn slowly over a period of 5 seconds. The samples were dried in a tared aluminum dish at $105^{\circ} \mathrm{C}$, and weighed to the nearest $0.0001 \mathrm{~g}$. The sand fraction was sieved from the remaining sediment after clay sampling. For the sand fraction a 270 mesh $(53 \mu \mathrm{m})$ sieve was used. The sand was washed into tared $50 \mathrm{ml}$ beakers, dried, and weighed. Silt content was estimated as the difference between the sum of the sand and clay, and the initial sample weight.

\footnotetext{
${ }^{2}$ Miller, W.P. and M. Miller. 1987. "A Micro-Pipette Method for Sediment Mechanical Analysis." Communications in Sediment Sci., Plant Anal. 18(1) 1-15.
} 


\section{Toxicity Characteristic Leaching Procedure Extraction (TCLP Test)}

Extraction solution

$0.1 \mathrm{M}$ glacial acetic acid

$0.0643 \mathrm{M} \mathrm{NaOH}$

$\mathrm{pH} 4.93$

Add $600 \mathrm{ml}$ of DI water to a $1 \mathrm{~L}$ volumetric flask. Add stir bar and stir. Add $5.7 \mathrm{ml}$ of glacial acetic acid (or acetic acid), $\mathrm{CH}_{3} \mathrm{CO}_{2} \mathrm{H}$. Add $64.4 \mathrm{ml}$ of $1 \mathrm{M} \mathrm{NaOH}$. Remove stir bar and bring up to $1 \mathrm{~L}$ with DI water. Measure $\mathrm{pH}$. Method

1. Label the centrifuge tubes according to the label list in the Table 5B. Add 2 grams dried apatite material and add $40 \mathrm{~mL}$ of TCLP extraction solution.

2. Put the centrifuge tubes on the rotating shaker for $18 \mathrm{hrs}$.

3. Pass through a disposable $0.45 \mu \mathrm{m}$ polycarbonate filter.

4. Acidify to $2 \% \mathrm{HNO}_{3}$; add $740 \mu \mathrm{m}$ concentrated $\mathrm{HNO}_{3}$ (this assumes a final sample volume of $37 \mathrm{~mL}$ )

5. Send samples for ICP analysis for 22 elements. 
Table 5B. Toxicity Characteristic Leaching Procedure Extraction (TCLP Test)

\begin{tabular}{|l|l|l|l|l|l|l|l|}
\hline $\begin{array}{l}\text { Number } \\
\text { ID }\end{array}$ & Product name/location & $\begin{array}{l}\text { A short } \\
\text { Name }\end{array}$ & Replicates & $\begin{array}{l}\text { Lab } \\
\text { Label }\end{array}$ & $\begin{array}{l}\text { Tube wt } \\
\text { [g] }\end{array}$ & $\begin{array}{l}\text { Sample net } \\
\text { Weight } \\
\text { [g] }\end{array}$ & $\begin{array}{l}\text { Volume } \\
\text { of } \\
\text { extraction } \\
\text { sol. } \\
{[\mathrm{mL}]}\end{array}$ \\
\hline 1 & & & & & & \\
\hline & Phosphate rock & PR-FL & 1 & $1-1$ & 11.7566 & 2.0026 & 40 \\
\hline 2 & Florida & & 2 & $1-2$ & 11.7087 & 2.0048 & 40 \\
\hline & Montana Natural Granulated & GRP-MT & 1 & $2-1$ & 11.7017 & 2.0029 & 40 \\
\hline 3 & Rock Phosphate, Montana & & 2 & $2-2$ & 11.7197 & 2.0064 & 40 \\
\hline & Spring Phosphate Powdered & PRP-ID & 1 & $3-1$ & 11.7368 & 2.0013 & 40 \\
\hline 4 & Rock Phosphate, Idaho & & 2 & $3-2$ & 11.7711 & 2.0045 & 40 \\
\hline & Volcanaphos- granulated rock & VCP-ID & 1 & $4-1$ & 11.7562 & 2.0038 & 40 \\
\hline 5 & phosphate, Idaho & & 2 & $4-2$ & 11.7241 & 2.0086 & 40 \\
\hline & Tennessee Brown Rock & BR-TN & 1 & $5-1$ & 11.7128 & 2.0033 & 40 \\
\hline 6 & Tennessee & & 2 & $5-2$ & 11.8609 & 2.0047 & 40 \\
\hline & Tennessee brown Rock & WPO- & 1 & $6-1$ & 11.7062 & 2.005 & 40 \\
\hline 7 & washed Phosphate Ore, TN & & 2 & $6-2$ & 11.7274 & 2.0026 & 40 \\
\hline & Phosphate rock-ore & WG-IPR & 1 & $7-1$ & 11.8101 & 2.0069 & 40 \\
\hline & Rasmussen Ridge Mine & & 2 & $7-2$ & 11.7006 & 2.0013 & 40 \\
\hline 8 & in Soda Spring, Idaho & & & & & & \\
\hline & North Carolina Apatite & NCA & 1 & $8-1$ & 11.7136 & 2.0026 & 40 \\
\hline 9 & & & 2 & $8-2$ & 11.7335 & 2.0066 & 40 \\
\hline & Biological Apatite & BA & 1 & $9-1$ & 11.7587 & 2.0023 & 40 \\
\hline & Blank & & 2 & $9-2$ & 11.7196 & 2.0076 & 40 \\
\hline & (extraction solution) & & & $0-1$ & 11.7317 & & 40 \\
\hline
\end{tabular}


WSRC-TR-2003-00579, REV. 0

\section{Laboratory Measurement of Zone-of-Influence (ZOI)}

\section{Vertical application of $\mathrm{Fe}(\mathrm{0})$ and apatite}

Objective: To determine horizontal (lateral) movement of P/and Fe in sediment from the TNX OD (B5) with vertical application of North Carolina Apatite, Biological Apatite and $\mathrm{Fe}(0)$ application

The experiment was performed in $60 \mathrm{ml}$ syringes; $4 \mathrm{~cm}$ of saturated sediment (B5, the TNX OD) was placed into the syringe and on the top of the sediment was placed a $1.1 \mathrm{~cm}$ layer of saturated amendment (apatite or $\mathrm{Fe}$ ). The syringes were sealed and placed vertically on the lab bench for a period of 3 months. On August 12, 2003 (after 3 months), the bottom of the syringe with the sample was cut off and the sample was gently pushed out and each $0.5 \mathrm{~cm}$ of pushed sample was collected individually. Each collected slice of sediment and amendment was digested by $\mathrm{HNO}_{3}, \mathrm{H}_{2} \mathrm{SO}_{4} / \mathrm{HCl}$ and the digested extracts were analyzed for total content of P or Fe by ICP-AES.

The experimental design:

Four treatments:

4 controls: $\quad$ only sediment (B5) $-4 \mathrm{~cm}$, (label: C-1 B5) only biological phosphate (BP) $-1.1 \mathrm{~cm}$ (label: C-2 BA) only North Carolina Apatite (NCA) - $1.1 \mathrm{~cm}$ (label: C-3 NCA) only $\mathrm{Fe}(0)-1.1 \mathrm{~cm}$ (Label: C-4 Fe(0))

Sediment B5 $(4 \mathrm{~cm})$ and BP $(1.1 \mathrm{~cm}) \times 5$ (Labels: 1-1B5/BP.....1-5B5/BA)

Sediment B5 $(4 \mathrm{~cm})$ and NCA $(1.1 \mathrm{~cm}) \times 4$ (Labels: 2-1 B5/NCA....2-4B5/NCA)

Sediment B5 $(4 \mathrm{~cm})$ and $\mathrm{Fe}(0)(1.1 \mathrm{~cm}) \times 4$ (Labels: $3-1 \mathrm{~B} 5 / \mathrm{Fe}(0) \ldots .3-4 \mathrm{~B} 5 / \mathrm{Fe}(0))$ 
WSRC-TR-2003-00579, REV. 0

Table 6B. Laboratory measurement of Zone-of-Influence (ZOI)

\begin{tabular}{|c|c|c|c|c|c|c|c|c|c|}
\hline \multirow[t]{3}{*}{ Label } & \multirow[t]{3}{*}{ Description } & \multicolumn{8}{|c|}{ Description of sub-samples* } \\
\hline & & \multicolumn{8}{|c|}{ Length $(\mathrm{cm}) /$ weight $(\mathrm{g})$} \\
\hline & & $\mathrm{A}$ & $\mathrm{b}$ & $\mathrm{c}$ & $\mathrm{d}$ & $\mathrm{e}$ & $\mathrm{f}$ & g & $\mathrm{h}$ \\
\hline & & $1 \mathrm{~cm}$ & $0.2 \mathrm{~cm}$ & $0.3 \mathrm{~cm}$ & $0.5 \mathrm{~cm}$ & $0.5 \mathrm{~cm}$ & $0.5 \mathrm{~cm}$ & $1 \mathrm{~cm}$ & $1 \mathrm{~cm}$ \\
\hline $1-1 \mathrm{~B} 5 / \mathrm{BP}$ & Sediment B5/ & & & & & & & & \\
\hline $1-2 \mathrm{~B} 5 / \mathrm{BP}$ & Biological Phosphate & & & & & & & & \\
\hline $1-3 \mathrm{~B} 5 / \mathrm{BP}$ & & & & & & & & & \\
\hline 1-4 B5/BP & & & & & & & & & \\
\hline $1-5 \mathrm{~B} 5 / \mathrm{BP}$ & & & & & & & & & \\
\hline 2-1 B5/NCA & Sediment B5/ & & & & & & & & \\
\hline 2-2 B5/NCA & North Carolina Apatite & & & & & & & & \\
\hline 2-3 B5/NCA & & & & & & & & & \\
\hline 2-4 B5/NCA & & & & & & & & & \\
\hline 3-1 $\mathrm{B} 5 / \mathrm{Fe}(0)$ & $\mathrm{Fe}(0)$ & & & & & & & & \\
\hline $3-2 \mathrm{~B} 5 / \mathrm{Fe}(0)$ & & & & & & & & & \\
\hline 3-3 $\mathrm{B} 5 / \mathrm{Fe}(0)$ & & & & & & & & & \\
\hline $3-4 \mathrm{~B} 5 / \mathrm{Fe}(0)$ & & & & & & & & & \\
\hline C-1 B5 & $4 \mathrm{~cm}$ & & & & & & & & \\
\hline C-2 BA & $1.1 \mathrm{~cm}$ & & & & & & & & \\
\hline C-3 NCA & $1.1 \mathrm{~cm}$ & & & & & & & & \\
\hline $\mathrm{C}-4 \mathrm{Fe}(0)$ & $1.1 \mathrm{~cm}$ & & & & & & & & \\
\hline
\end{tabular}

* Each sample was divided as the following: $1 \mathrm{~cm}$ slice (amendment - slice a); $0.2 \mathrm{~cm}$ slice (interlayer amendment/sediment B5 - slice b); $0.3 \mathrm{~cm}$ slice of sediment (c); $0.5 \mathrm{~cm}$ slice of sediment (d, e, and f); $1.0 \mathrm{~cm}$ slice of sediment $(\mathrm{g}$ and $\mathrm{h})$. 
WSRC-TR-2003-00579, REV. 0

Appendix C: Work Insturctions and Additional Results for the Field Study 


\section{Work Instructions}

Sediment Amendment Study at TNX OD Outfall Delta

ERD-EN-2002-0099 Rev. 0

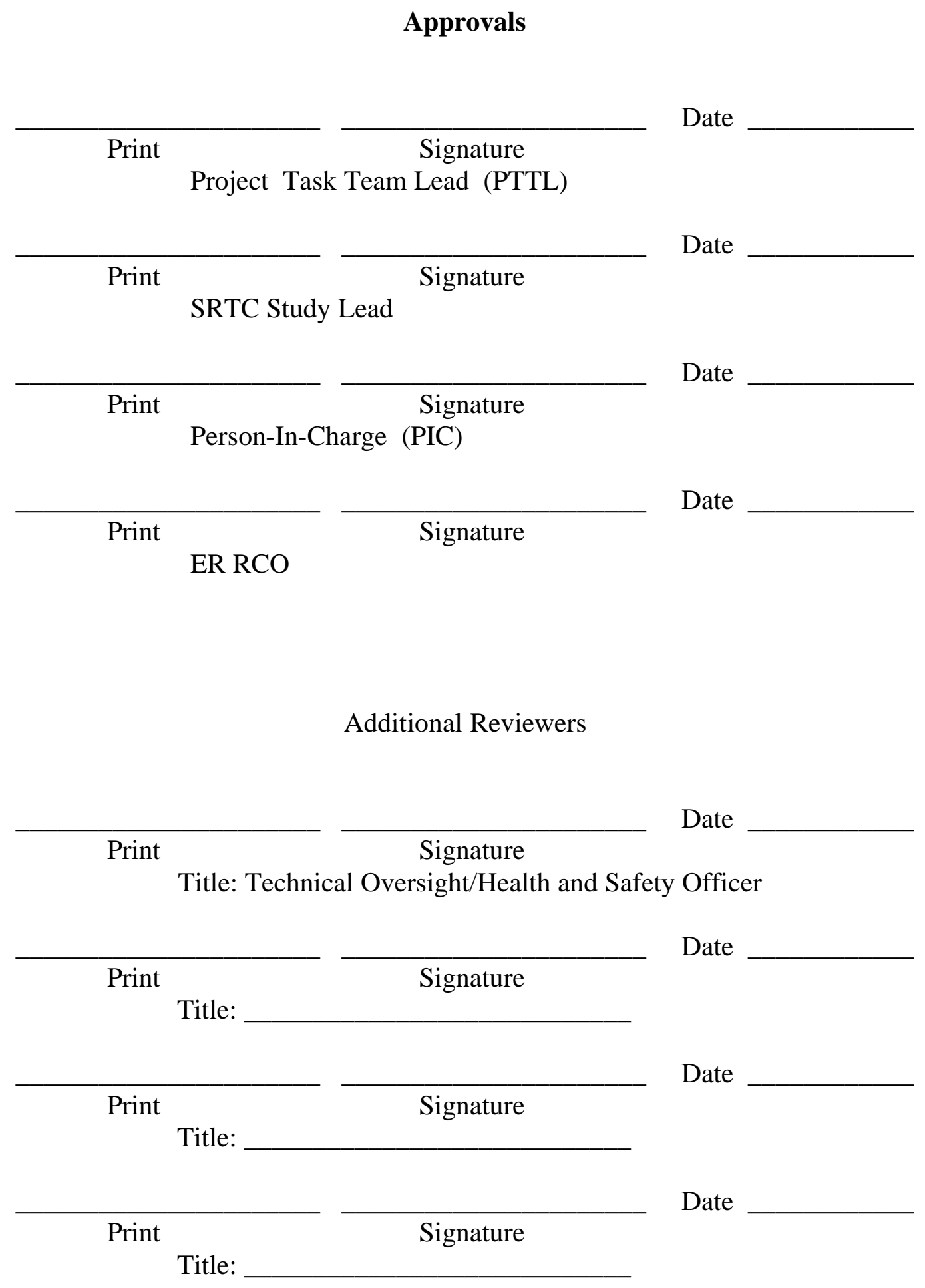




\section{PURPOSE}

These Work Instructions identify and describe Scope, Safety, Special Instructions, Prerequisites, Work Steps, Radiological Action Steps and clearance activities for performing the study as described in the "Technical Task Plan for Proof-of-principle Laboratory Study of Effects of Sediment Amendments on Contaminant Immobilization in TNX Outfall Delta Sediments and Zone of Influence Field Treatability Study of Sediment Amendments in TNX Outfall Delta Sediments."

\section{SCOPE}

Personnel will perform sediment sampling and introduce amendments to the sediment at depths not exceeding 12 inches in accordance with the "Technical Task Plan for Proof-ofprinciple Laboratory Study of Effects of Sediment Amendments on Contaminant Immobilization in TNX Outfall Delta Sediments and Zone of Influence Field Treatability Study of Sediment Amendments in TNX Outfall Delta Sediments." Radiological surveillance will be provided to ensure worker protective measures are adequate and to ensure radiological controls are heeded. The location of this study is at the TNX Outfall Delta the area is posted as a Sediment Contamination Area (SCA) and a Controlled Area.

\section{SAFETY}

- Personnel shall comply with all applicable safety standards, procedures and practices for the job and area.

- A pre-job briefing is required prior to performing any work. These work instructions will be used for the initial sampling and study set up and subsequent sampling for study results, a pre-job briefing will be required for each and every event.

- Worker notification should be via radio to KIJ5 or equivalent. Other equivalent means of communication may be employed to achieve emergency notification.

- Personnel will maintain awareness of uneven terrain, embankments, slopes, loose rocks and tripping hazards.

- Maintain awareness of poisonous plants, animals and insects.

- Additional safety precautions shall be taken as noted in the Work Package Documentation and Site Specific Health and Safety Plan (SSHASP) (Q-SHP-2002-00037).

- Workers shall be cognizant to the symptoms of heat stress and maintain awareness of workers and themselves for the symptoms of heat stress.

\section{PREREQUSITIES}

- Pre-Job Briefing.

- RCO is to be notified prior to entry to the SCA.

- Radiological Worker Training II for all workers donning radiological protective clothing.

- Radiological Work Permit (RWP) 02ER-001 for Radioactive Material Area (RMA) access (if needed) and Restricted Radiological Use Only (RRUO) vehicle operation

- Radiological Work Permit (RWP) 02ER-002 for tasks requiring donning of Radiological Use gloves, such as sediment sampling, withdrawing items from subsurface sediments (i.e., items that have penetrated the ground surface), and decontamination of tools. 
- An RMA will be established or designated if required. The RCO FM approval for a new RMA must be obtained prior to installation.

- Rad launderable Personal Protective Equipment (PPE) to include rubber gloves and rubber shoecovers or boots (for wet field conditions).

- PPE shall be staged near the work site in advance.

- Receptacles shall be established to receive radiological protective clothing. Sedimented/used Radiological Use PPE containers will be radiologically surveyed, labeled/tagged and removed from the area and transported to the ERD Laundry Station each day. No radiological laundry will be left unattended in the field.

\section{SPECIAL INSTRUCTIONS}

- Hazardous Material Transportation Representative (HMTR) approval for transport of samples field screened at $<2 \mathrm{nCi} / \mathrm{g}$ total alpha/beta/gamma activity has been obtained for transport of radiological materials on site roads and on and across Highway 125. HMTR must be contacted if total activity $>2 \mathrm{nCi} / \mathrm{g}$.

- Stage supplies and equipment outside the SCA. Do not bring unnecessary items into the SCA.

- Items, materials or samples designated or suspected as being radioactive material shall be stored or staged in an RMA or under continuous control by a qualified radiological worker.

- Continuous RCO radiological surveillance is required for sampling or withdrawing items from subsurface sediments (i.e., items that have penetrated the ground surface).

- RCO is to be notified prior to moving the RRUO vehicle outside a controlled area.

- Personnel shall be monitored by RCO after removing radiological PPE.

- If unexpected radiological conditions are encountered or radiological posting boundaries must be modified, RCO shall notify RCO First Line Manager and PIC of situation immediately, after a safe condition has been established.

- RCO shall perform radiological surveys of the equipment for the purpose of uncontrolled radiological release at conclusion of job evolution. Surfaces of equipment shall be dry, industrially clean and free of vegetation and sediment prior to radiological survey.

\section{CONTACTS}

\begin{tabular}{|l|l|ll|}
\hline Dennis Stapleton & Project Task Team Lead & $952-6652$ & Beeper 16014 \\
\hline Angela Daniel & Person In Charge (PIC) & $952-6534$ Beeper 19142 \\
\hline Dan Kaplan & SRTC Study Lead & $725-2363$ & \\
\hline Mark Mansfield & Safety \& Health Lead & $952-6484$ Beeper 11088 \\
\hline Darren Gillis & Waste Management Lead & $952-6504$ Beeper 15675 \\
\hline Greg Joyner & ER RCO First Line Manager & $952-7927$ Beeper 11895 \\
\hline Cathy Madore & ERD SIRIM Coordinator & $952-6656 \quad$ Beeper 19365 \\
\hline Laura Bagwell & Technical Oversight/Health \& Safety Officer & $952-6787$ Beeper 17370 \\
\hline Sallie Cooks & RRUO Vehicle Operator (sample delivery) & $952-6704$ Beeper 14728 \\
\hline
\end{tabular}


WSRC-TR-2003-00579, REV. 0

\section{WORK STEPS AND INSTRUCTIONS}

\begin{tabular}{|c|c|}
\hline Work Step & Work Instructions for Outfall Delta Sampling \\
\hline 1 & $\begin{array}{l}\text { If not previously performed, request approval from RCO Facility Manager for } \\
\text { RRUO designation of sample transport vehicle in accordance with 5Q1.1- } \\
522 \text {. Ensure vehicle RRUO tag is current. Establish laundry and waste (if } \\
\text { waste is generated) receptacles at the work site. }\end{array}$ \\
\hline \multirow[t]{2}{*}{2} & $\begin{array}{l}\text { Contact RCO for coverage prior to any intrusive work in the Outfall Delta } \\
\text { SCA. }\end{array}$ \\
\hline & $\begin{array}{l}\text { The statistical design of this experiment is a randomized complete block } \\
\text { (Figure 1). There are } 3 \text { replicates (blocks) and } 7 \text { treatments (control, } \\
\text { horizontal } \mathrm{Fe}(0) \text {, vertical } \mathrm{Fe}(0) \text {, horizontal apatite, vertical apatite, horizontal } \\
\text { apatite }+\mathrm{Fe}(0) \text {, and a vertical apatite }+\mathrm{Fe}(0) \text {. The experiment will be located } \\
\text { between markers A- } 5 \text { and A- } 6 \text { in a flat, vegetation-free area of } 480-\mathrm{x} 180-\mathrm{cm} \text {. } \\
\text { Mark the } 4 \text { corners of experimental plots with permanent stakes. Use flags to } \\
\text { locate corners of } 30 \mathrm{x} 30 \mathrm{~cm} \text { plots. A } 45-\mathrm{cm} \text { buffer zone (area between } \\
\text { treatments) will exist between all plots. }\end{array}$ \\
\hline 3 & $\begin{array}{l}\text { Obtain and prepare samples in accordance with the Technical Task Plan. } \\
\text { Using a 3/4-inch diameter stainless steel auger with a plastic liner, recover a } \\
30 \text {-cm core from the center of the plot. Remove the liner from the auger, put } \\
\text { end caps and label liner as described in Table } 1 \text {. Before proceeding to next } \\
\text { location, rinse dirt off auger by squirting distilled-water onto the auger and } \\
\text { rubbing the auger with gloves. Include } 2 \text { duplicates, i.e., } 1 \text { duplicate for about } \\
\text { every } 10 \text { samples. Include } 1 \text { field blank control. All samples will be logged } \\
\text { in the SRTC Field Sample Logbook. }\end{array}$ \\
\hline $\begin{array}{l}\text { RCO Action } \\
\text { Step } 1\end{array}$ & $\begin{array}{l}\text { Perform radiological surveys during sampling and when items are withdrawn } \\
\text { from the sediment subsurface. Ensure samples are packaged in accordance } \\
\text { with radioactive material requirements. Identify and label accordingly. If } \\
\text { contamination or radioactivity is found above RWP suspension guides or } \\
\text { above RBA posting limits, inform workers to establish a safe condition, } \\
\text { suspend work, monitor personnel, post in accordance with procedure 5Q1.2. } \\
518 \text { and inform RCO Management and PIC immediately. }\end{array}$ \\
\hline 4 & $\begin{array}{l}\text { Apply treatments to plots as described in Table } \mathbf{2 ~ C} \text {. Repeat work step 3, } \\
\text { RCO Action Step 1, and work step } 4 \text { as necessary to accomplish all sampling } \\
\text { and sediment amendment application as described in Tables } \mathbf{1 C} \text { and } \mathbf{2 C} \text {. }\end{array}$ \\
\hline 5 & $\begin{array}{l}\text { Items to be removed from the sampling area (for unconditional release) will } \\
\text { be prepared for radiological survey by ensuring they are industrially clean, all } \\
\text { assessable areas have been exposed and unconditional release documentation } \\
\text { has been prepared. Rinsate may fall to ground at sample location. Return } \\
\text { sample area to pre-sampling condition. Dispose of any generated waste in } \\
\text { accordance with Waste Management Requirements. }\end{array}$ \\
\hline $\begin{array}{l}\text { RCO Action } \\
\text { Step } \\
2\end{array}$ & $\begin{array}{l}\text { RCO shall perform radiological release survey of equipment (including } \\
\text { vehicles, if applicable) and materials for the purpose of unconditional } \\
\text { radiological release. Inform work group of radiological status of items or }\end{array}$ \\
\hline
\end{tabular}


WSRC-TR-2003-00579, REV. 0

\begin{tabular}{|l|l|}
\hline & equipment. \\
\hline 6 & $\begin{array}{l}\text { If radiological surveys so indicate, decontaminate items and equipment. } \\
\text { Dispose of any generated waste in accordance with Waste Management } \\
\text { Requirements. }\end{array}$ \\
\hline 7 & $\begin{array}{l}\text { Repeat work step 5, RCO Action Step 2 and work step } 6 \text { until all items and } \\
\text { equipment (including RRUO vehicles, if applicable) meet the criteria for } \\
\text { unconditional release. If unconditional release criteria can not be met, inform } \\
\text { RCO management and PIC of radiological status of item or equipment. } \\
\text { NOTE: Items not meeting the criteria for unconditional radiological release } \\
\text { shall be contained and identified as radioactive material and stored in an } \\
\text { RMA. It may be necessary to move so designated radioactive material to } \\
\text { another site location with an RRUO vehicle. }\end{array}$ \\
\hline RCO Action & $\begin{array}{l}\text { RCO shall perform radiological surveys as necessary with the goal of } \\
\text { returning the sample area to its radiological status prior to sampling (SCA). } \\
\text { Inform RCO management and PIC of radiological conditions before changing } \\
\text { posting and upon completion of this reposting. IF conditions for SCA } \\
\text { Radiological Posting can not be met, inform RCO FLM and PIC of } \\
\text { radiological status and conditions of area. }\end{array}$ \\
\hline 8 & $\begin{array}{l}\text { Ensure all equipment, materials, waste (if any) or other items has been } \\
\text { removed from the SCA unless stored in an approved RMA. }\end{array}$ \\
\hline
\end{tabular}


WSRC-TR-2003-00579, REV. 0

Figure 1 C. Experimental Plot Design

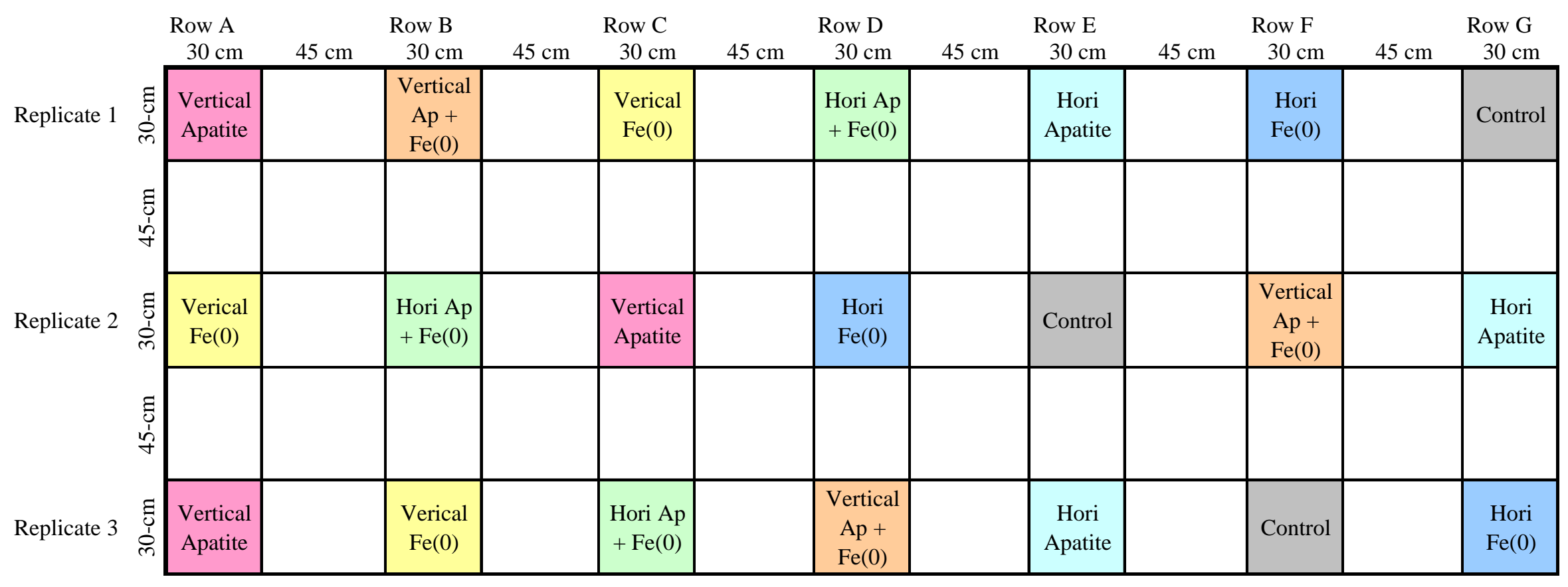


WSRC-TR-2003-00579, REV. 0

Table 1 C. Sample Identification

\begin{tabular}{|l|c|l|}
\hline Sample Code & $\begin{array}{l}\text { EMS/EGG Sample } \\
\text { Logbook Number }\end{array}$ & Sample Description \\
\hline C-I-0 & 900101 & Control, Replicate 1, Time Zero \\
\hline C-II-0 & 900102 & Control, Replicate 2, Time Zero \\
\hline C-III-0 & 900103 & Control, Replicate 3, Time Zero \\
\hline HA-I-0 & 900104 & Horizontal Apatite, Replicate 1, Time Zero \\
\hline HA-II-0 & 900105 & Horizontal Apatite, Replicate 2, Time Zero \\
\hline HA-III-0 & 900106 & Horizontal Apatite, Replicate 3, Time Zero \\
\hline HAF-I-0 & 900107 & Horizontal Apatite + Fe(0), Replicate 1, Time Zero \\
\hline HAF-II-0 & 900108 & Horizontal Apatite + Fe(0), Replicate 2, Time Zero \\
\hline HAF-II-0-dup & 900109 & Horizontal Apatite + Fe(0), Rep. 2, Time Zero, Duplicate \\
\hline HAF-III-0 & 900110 & Horizontal Apatite + Fe(0), Replicate 3, Time Zero \\
\hline HF-I-0 & 900111 & Horizontal Fe(0), Replicate 1, Time Zero \\
\hline HF-II-0 & 900112 & Horizontal Fe(0), Replicate 2, Time Zero \\
\hline HF-III-0 & 900113 & Horizontal Fe(0), Replicate 3, Time Zero \\
\hline VA-I-0 & 900114 & Vertical Apatite, Replicate 1, Time Zero \\
\hline VA-II-0 & 900115 & Vertical Apatite, Replicate 2, Time Zero \\
\hline VA-III-0 & 900116 & Vertical Apatite, Replicate 3, Time Zero \\
\hline VAF-I-0 & 900117 & Vertical Apatite + Fe(0), Replicate 1, Time Zero \\
\hline VAF-II-0 & 900118 & Vertical Apatite + Fe(0), Replicate 2, Time Zero \\
\hline VAF-III-0 & 900119 & Vertical Apatite + Fe(0), Replicate 3, Time Zero \\
\hline VF-I-0 & 900120 & Vertical Fe(0), Replicate 1, Time Zero \\
\hline VF-II-0 & 900121 & Vertical Fe(0), Replicate 2, Time Zero \\
\hline VF-II-0-dup & 900122 & Vertical Fe(0), Replicate 3, Time Zero \\
\hline VF-III-0 & 900123 & Vertical Fe(0), Replicate 3, Time Zero \\
\hline Field blank & 900124 & $\begin{array}{l}\text { Field Blank: empty sample containers; no sediment } \\
\text { collection; check on cross contamination and aerial } \\
\text { deposition }\end{array}$ \\
\hline
\end{tabular}


WSRC-TR-2003-00579, REV. 0

Table 2C. Treatment Description

\begin{tabular}{|l|l|}
\hline Treatment & Description \\
\hline Control & Add nothing \\
\hline Horizontal Apatite & Surface apply 163-g Apatite \\
\hline Horizontal Apatite $+\mathrm{Fe}(0)$ & Surface apply 163-g Apatite + 163-g Fe(0) \\
\hline Horizontal Fe(0) & Surface apply 163-g Fe(0) \\
\hline Vertical Apatite & $\begin{array}{l}\text { Pack 115 g Apatite into existing "sample" hole (3/4-diameter, 30- } \\
\text { cm deep) using stainless steel rod. }\end{array}$ \\
\hline Vertical Apatite + Fe(0) & $\begin{array}{l}\text { Pack 115 g Apatite and 400-g Fe(0) into two existing "sample" } \\
\text { holes (each 3/4-diameter, 30-cm deep) using stainless steel rod }\end{array}$ \\
\hline Vertical Fe(0) & $\begin{array}{l}\text { Pack 400-g Fe(0) into existing "sample" hole (3/4-diameter, 30-cm } \\
\text { deep) using stainless steel rod }\end{array}$ \\
\hline
\end{tabular}


WSRC-TR-2003-00579, REV. 0

Table 3C. Sediment samples taken from the TNX OD before the application of the amendments

\begin{tabular}{llllll}
\hline Treatment & $\begin{array}{l}\text { Sample } \\
\text { code }\end{array}$ & $\begin{array}{l}\text { EMS/EG } \\
\text { G } \\
\text { sample } \\
\text { Logbook }\end{array}$ & $\begin{array}{l}\text { Sample } \\
\text { length } \\
\text { [inch }\end{array}$ & $\begin{array}{l}\text { Hole } \\
\text { depth } \\
\text { vertical } \\
\text { treatment }\end{array}$ & $\begin{array}{l}\text { Amount of } \\
\text { applied } \\
\text { amendment }\end{array}$ \\
\hline Control & & & & 0 \\
Horizontal apatite & C-I-0 & 900101 & & & 163 \\
Horizontal Fe(0) & HF-I-0 & 900104 & $31 / 2$ & & 163 \\
Control & C-II-0 & 900111 & $41 / 2$ & & 0 \\
Vertical Apatite \& Fe(0) & VAF-I-0 & 9001117 & $31 / 2$ & 5 & $62.6 \mathrm{~A} / 219 \mathrm{Fe}$ \\
Horizontal Fe(0) & HF-II-0 & 900112 & $23 / 4$ & & 163 \\
Horizontal Apatite & HA-II-0 & 900115 & $31 / 2$ & & 163 \\
Control & C-III-0 & 900103 & $21 / 2$ & & 0 \\
Horizontal apatite & HA-III-0 & 900106 & $31 / 4$ & & 163 \\
Vertical Apatite \& Fe(0) VAF-II-0 & 900118 & $31 / 2$ & $31 / 2$ & $41.7 \mathrm{~A} / 145.9 \mathrm{Fe}$ \\
Horizontal Fe(0) & HF-III-0 & 900113 & 3 & & 163 \\
Horizontal Apatite \& & HAF-I-0 & 900107 & $37 / 8$ & & $163 \mathrm{~A} \& 163 \mathrm{Fe}$ \\
Fe(0) & & & & & \\
Vertical Fe(0) & VF-I-0 & 900120 & $27 / 8$ & 4 & 400 \\
Vertical Apatite & VA-I-0 & 900114 & $25 / 8$ & 4 & 58.6 \\
Horizontal Apatite \& & HAF-II-0 & 900108 & $41 / 4$ & & $163 \mathrm{~A} \& 163 \mathrm{Fe}$ \\
Fe(0) & & & & & \\
Vertical Fe(0) & VF-II-0 & 900121 & 3 & $41 / 8$ & 400 \\
Horizontal Apatite \& & HAF-III-0 & 900109 & $25 / 8$ & & $163 \mathrm{~A} \& 163 \mathrm{Fe}$ \\
Fe(0) & & & & & \\
& & 900110 & $25 / 8$ & & $115 \mathrm{~A} / 400 \mathrm{Fe}$ \\
Horizontal Apatite \& & HAF-IV-0 & 900119 & $31 / 8$ & & \\
Fe(0) & & & & & \\
Vertical Apatite & VA-II-0 & 900115 & $27 / 8$ & $37 / 8$ & $542 / 7$ \\
Vertical Fe(0) & VF-III-0 & 900122 & $33 / 8$ & & 400 \\
Vertical Apatite & VA-III-0 & 900123 & $33 / 8$ & $51 / 8$ & \\
Vertical Apatite \&Fe(0) & VAF-III-0 & 900116 & $67 / 8$ & $91 / 2$ & 115 \\
Field blank & & $27 / 8$ & & $22.7 \mathrm{~A} / 79.3 \mathrm{Fe}$ \\
\hline
\end{tabular}




\section{Measurement of Zone-of-Influence (ZOI) based on the field samples}

The objective of this study was to determine the ZOI of $\mathrm{Fe}(0)$ and two types of apatite in the TNX OD sediment after 13 months of their application.

Table 4C. Treatment description

\begin{tabular}{|c|c|c|}
\hline $\begin{array}{l}\text { Application } \\
\text { date }\end{array}$ & Treatment & Description \\
\hline $8 / 8 / 02$ & Control & Add nothing \\
\hline $8 / 8 / 02$ & $\begin{array}{l}\text { Horizontal Biological } \\
\text { Phosphate (BP) }\end{array}$ & Surface apply $163 \mathrm{~g}$ of BP \\
\hline $8 / 8 / 02$ & Horizontal $\mathrm{BP}+\mathrm{Fe}(0)$ & $\begin{array}{l}\text { Surface apply of } 163 \mathrm{~g} \text { of BP and } 163 \mathrm{~g} \text { of } \\
\mathrm{Fe}(0)\end{array}$ \\
\hline $8 / 8 / 02$ & Horizontal $\mathrm{Fe}(0)$ & Surface apply of $163 \mathrm{~g} \mathrm{Fe}(0)$ \\
\hline $8 / 22 / 02$ & $\begin{array}{l}\text { Horizontal North Carolina } \\
\text { Apatite NCA }\end{array}$ & Surface apply of $163 \mathrm{~g}$ of NCA \\
\hline $11 / 7 / 02$ & Horizontal NCA + Fe (0) & $\begin{array}{l}\text { Surface supply of } 163 \mathrm{~g} \text { of NCA and } 163 \text { of } \\
\mathrm{Fe}(0)\end{array}$ \\
\hline $8 / 8 / 02$ & Vertical BP & Packed $59 \mathrm{~g} / 54 \mathrm{~g} / 115 \mathrm{~g}$ of BP into a hole \\
\hline $8 / 8 / 02$ & Vertical $\mathrm{Ba}+\mathrm{Fe}(0)$ & $\begin{array}{l}\text { Packed } 63 \mathrm{BP} / 219 \mathrm{Fe}(0) ; 42 \mathrm{BA} / 146 \mathrm{Fe}(0) \\
\text { 23BA/79Fe(0) }\end{array}$ \\
\hline $8 / 8 / 02$ & Vertical Fe (0) & Packed $400 \mathrm{~g} / 400 \mathrm{~g} / 400 \mathrm{~g}$ of $\mathrm{Fe}(0)$ \\
\hline $8 / 22 / 02$ & Vertical NCA & Packed $210 \mathrm{~g} / 111 \mathrm{~g} / 114 \mathrm{~g}$ of $\mathrm{NCA}$ \\
\hline $11 / 7 / 02$ & Vertical $\mathrm{NCA}+\mathrm{Fe}(0)$ & Packed \\
\hline
\end{tabular}

Treatments with horizontal application of $\mathrm{Fe}(0)$ and two types of apatite (North Carolina Apatite and Biological Phosphate):

There are 5 horizontal treatments (Table 1) with three replicates in each (total number of plots 15) and one control treatment (three replicates).

Sediment samples from the horizontal treatments were collected with a sediment sampler into plastic sleeves ( $\sim$ inch diameter; sampling depth $\sim 5$ inches).

The sediment samples were collected from the center of each plot (next to the hole after the first sediment sampling). Collected sediments were sliced (every $1 \mathrm{~cm}$ ) and each slice was analyzed for the following:

$\mathrm{pH}$

Total concentrations of $\mathrm{P}$ or $\mathrm{Fe}$ in the extracts from a total digestion with $\mathrm{HNO}_{3} / \mathrm{H}_{2} \mathrm{SO}_{4} / \mathrm{HCl}$ were analyzed by ICP-AES.

Treatments with vertical application of $\mathrm{Fe}(0)$ and two types of apatite (North Carolina Apatite and Biological Phosphate):

There are 5 vertical treatments (Table 1) with three replicates in each (total number of plots is 15). Sediment samples from these treatments were collected from the center of each plot (next to the hole after the first sediment sampling) using a sediment profiler (7" deep x 4" wide and 1" thick). The collected sediment profile was divided vertically into $1 \mathrm{~cm}$ wide strips. Each strip was analyzed for $\mathrm{pH}$ and $\mathrm{P}$ or Fe accordingly to the treatment. 
Table 5C. Sampling Plan After 13 Months of Amendments Application

\begin{tabular}{|c|c|c|c|c|c|}
\hline $\begin{array}{l}\text { SRTC } \\
\text { ID\# }\end{array}$ & $\begin{array}{l}\text { Treatment } \\
\text { Label }\end{array}$ & Amendment & $\begin{array}{l}\text { Type of } \\
\text { Application }\end{array}$ & Sub-sample Labels & Sampling Tool \\
\hline 1 & Control-I & None & Horizontal & $1 \mathrm{~A}, 1 \mathrm{~B}, 1 \mathrm{C}^{*}$ & $\begin{array}{l}\text { sediment } \\
\text { sampler }\end{array}$ \\
\hline 2 & Control-II & None & Horizontal & $2 \mathrm{~A}, 2 \mathrm{~B}, 2 \mathrm{C}$ & $\begin{array}{l}\text { sediment } \\
\text { sampler }\end{array}$ \\
\hline 3 & Control-III & None & Horizontal & $3 \mathrm{~A}, 3 \mathrm{~B}, 3 \mathrm{C}$ & $\begin{array}{l}\text { sediment } \\
\text { sampler }\end{array}$ \\
\hline 4 & Control-I & None & Vertical & $4 \mathrm{a}, 4 \mathrm{~b}, 4 \mathrm{c}, 4 \mathrm{~d}, \ldots$ & $\begin{array}{l}\text { sediment } \\
\text { profiler }\end{array}$ \\
\hline 5 & Control-II & None & Vertical & $5 \mathrm{a}, 5 \mathrm{~b}, 5 \mathrm{c}, 5 \mathrm{~d} \ldots$ & $\begin{array}{l}\text { sediment } \\
\text { profiler }\end{array}$ \\
\hline 6 & Control-III & None & Vertical & $6 a, 6 b, 6 c, 6 d \ldots$ & $\begin{array}{l}\text { sediment } \\
\text { profiler }\end{array}$ \\
\hline 7 & H-NCA-I & North Carolina Apatite & Horizontal & $7 \mathrm{~A}, 7 \mathrm{~B}, 7 \mathrm{C}$ & $\begin{array}{l}\text { sediment } \\
\text { sampler }\end{array}$ \\
\hline 8 & H-NCA-II & North Carolina Apatite & Horizontal & $8 \mathrm{~A}, 8 \mathrm{~B}, 8 \mathrm{C}$ & $\begin{array}{l}\text { sediment } \\
\text { sampler }\end{array}$ \\
\hline 9 & H-NCA-III & North Carolina Apatite & Horizontal & $9 \mathrm{~A}, 9 \mathrm{~B}, 9 \mathrm{C}$ & $\begin{array}{l}\text { sediment } \\
\text { sampler }\end{array}$ \\
\hline 10 & V-NCA-I & North Carolina Apatite & Vertical & $10 \mathrm{a}, 10 \mathrm{~b}, 10 \mathrm{c}, \ldots$ & $\begin{array}{l}\text { sediment } \\
\text { profiler }\end{array}$ \\
\hline 11 & V-NCA-II & North Carolina Apatite & Vertical & $11 \mathrm{a}, 11 \mathrm{~b}, 11 \mathrm{c} \ldots$ & $\begin{array}{l}\text { sediment } \\
\text { profiler }\end{array}$ \\
\hline 12 & V-NCA-III & North Carolina Apatite & Vertical & $12 \mathrm{a}, 12 \mathrm{~b}, 12 \mathrm{c} \ldots$ & $\begin{array}{l}\text { sediment } \\
\text { profiler }\end{array}$ \\
\hline
\end{tabular}


WSRC-TR-2003-00579, REV. 0

\begin{tabular}{|c|c|c|c|c|c|}
\hline 13 & H-BP-I & Biological Phosphate & Horizontal & $13 \mathrm{~A}, 13 \mathrm{~B}, 13 \mathrm{C}$ & $\begin{array}{l}\text { sediment } \\
\text { sampler }\end{array}$ \\
\hline 14 & H-BP-II & Biological Phosphate & Horizontal & $14 \mathrm{~A}, 14 \mathrm{~B}, 14 \mathrm{C}$ & $\begin{array}{l}\text { sediment } \\
\text { sampler }\end{array}$ \\
\hline 15 & H-BP-III & Biological Phosphate & Horizontal & $15 \mathrm{~A}, 15 \mathrm{~B}, 15 \mathrm{C}$ & $\begin{array}{l}\text { sediment } \\
\text { sampler }\end{array}$ \\
\hline 16 & V-BP-I & Biological Phosphate & Vertical & $16 a, 16 b, 16 c \ldots$ & $\begin{array}{l}\text { sediment } \\
\text { profiler }\end{array}$ \\
\hline 17 & V-BP-II & Biological Phosphate & Vertical & $17 \mathrm{a}, 17 \mathrm{~b}, 17 \mathrm{c} \ldots$ & $\begin{array}{l}\text { sediment } \\
\text { profiler }\end{array}$ \\
\hline 18 & V-BP-III & Biological Phosphate & Vertical & $18 \mathrm{a}, 18 \mathrm{~b}, 18 \mathrm{c} \ldots$ & $\begin{array}{l}\text { sediment } \\
\text { profiler }\end{array}$ \\
\hline 19 & H-Fe-I & $\mathrm{Fe}(0)$ & Horizontal & 19A, 19B. 19C & $\begin{array}{l}\text { sediment } \\
\text { sampler }\end{array}$ \\
\hline 20 & $\mathrm{H}-\mathrm{Fe}-\mathrm{II}$ & $\mathrm{Fe}(0)$ & Horizontal & $20 \mathrm{~A}, 20 \mathrm{~B}, 20 \mathrm{C}$ & $\begin{array}{l}\text { sediment } \\
\text { sampler }\end{array}$ \\
\hline 21 & $\mathrm{H}-\mathrm{Fe}-\mathrm{III}$ & $\mathrm{Fe}(0)$ & Horizontal & $21 \mathrm{~A}, 21 \mathrm{~B}, 21 \mathrm{C}$ & $\begin{array}{l}\text { sediment } \\
\text { sampler }\end{array}$ \\
\hline 22 & V-Fe-I & $\mathrm{Fe}(0)$ & Vertical & $22 \mathrm{a}, 22 \mathrm{~b}, 22 \mathrm{c} \ldots$ & $\begin{array}{l}\text { sediment } \\
\text { profiler }\end{array}$ \\
\hline 23 & V-Fe-II & $\mathrm{Fe}(0)$ & Vertical & $23 a, 23 b, 23 c \ldots$ & $\begin{array}{l}\text { sediment } \\
\text { profiler }\end{array}$ \\
\hline 24 & V-Fe-III & $\mathrm{Fe}(0)$ & Vertical & $24 a, 24 b, 24 c \ldots$ & $\begin{array}{l}\text { sediment } \\
\text { profiler }\end{array}$ \\
\hline 25 & H-NCA/Fe-I & North Carolina Apatite/Fe(0) & Horizontal & $25 \mathrm{~A}, 25 \mathrm{~B}, 25 \mathrm{C}$ & $\begin{array}{l}\text { sediment } \\
\text { sampler }\end{array}$ \\
\hline 26 & H-NCA/Fe-II & North Carolina Apatite/Fe(0) & Horizontal & $26 \mathrm{~A}, 26 \mathrm{~B}, 26 \mathrm{C}$ & $\begin{array}{l}\text { sediment } \\
\text { sampler }\end{array}$ \\
\hline 27 & H-NCA/Fe-III & North Carolina Apatite/Fe(0) & Horizontal & $27 \mathrm{~A}, 27 \mathrm{~B}, 27 \mathrm{C}$ & $\begin{array}{l}\text { sediment } \\
\text { sampler }\end{array}$ \\
\hline
\end{tabular}


WSRC-TR-2003-00579, REV. 0

\begin{tabular}{|c|c|c|c|c|c|}
\hline 28 & V-NCA/Fe-I & North Carolina Apatite/Fe(0) & Vertical & $28 \mathrm{a}, 28 \mathrm{~b}, 28 \mathrm{c} \ldots$ & $\begin{array}{l}\text { sediment } \\
\text { profiler }\end{array}$ \\
\hline 29 & $\mathrm{~V}-\mathrm{NCA} / \mathrm{Fe}-\mathrm{II}$ & North Carolina Apatite/Fe(0) & Vertical & $29 a, 29 b, 29 c \ldots$ & $\begin{array}{l}\text { sediment } \\
\text { profiler }\end{array}$ \\
\hline 30 & V-NCA/Fe-III & North Carolina Apatite/Fe(0) & Vertical & $30 \mathrm{a}, 30 \mathrm{~b}, 30 \mathrm{c} \ldots$ & $\begin{array}{l}\text { sediment } \\
\text { profiler }\end{array}$ \\
\hline 31 & H-BP/Fe-I & Biological Phosphate/Fe(0) & Horizontal & $31 \mathrm{~A}, 31 \mathrm{~B}, 31 \mathrm{C}$ & $\begin{array}{l}\text { sediment } \\
\text { sampler }\end{array}$ \\
\hline 32 & H-BP/Fe-II & Biological Phosphate/Fe(0) & Horizontal & $32 \mathrm{~A}, 32 \mathrm{~B}, 32 \mathrm{C}$ & $\begin{array}{l}\text { sediment } \\
\text { sampler }\end{array}$ \\
\hline 33 & $\mathrm{H}-\mathrm{BP} / \mathrm{Fe}-\mathrm{III}$ & Biological Phosphate/Fe(0) & Horizontal & $33 \mathrm{~A}, 33 \mathrm{~B}, 33 \mathrm{C}$ & $\begin{array}{l}\text { sediment } \\
\text { sampler }\end{array}$ \\
\hline 34 & V-BP/Fe-I & Biological Phosphate/Fe(0) & Vertical & $34 \mathrm{a}, 34 \mathrm{~b}, 34 \mathrm{c} \ldots \ldots$ & $\begin{array}{l}\text { sediment } \\
\text { profiler }\end{array}$ \\
\hline 35 & V-BP/Fe-II & Biological Phosphate/Fe(0) & Vertical & $35 a, 35 b, 35 c \ldots$ & $\begin{array}{l}\text { sediment } \\
\text { profiler }\end{array}$ \\
\hline 36 & V-BP/Fe-III & Biological Phosphate/Fe(0) & Vertical & $36 a, 36 b, 36 c \ldots$ & $\begin{array}{l}\text { sediment } \\
\text { profiler }\end{array}$ \\
\hline 37 & H-NCA-I d* & North Carolina Apatite & Horizontal & $37 \mathrm{~A}, 37 \mathrm{~B}, 37 \mathrm{C}$ & $\begin{array}{l}\text { sediment } \\
\text { sampler }\end{array}$ \\
\hline 38 & H-NCA/Fe-IId & North Carolina Apatite/Fe(0) & Horizontal & $38 \mathrm{~A}, 38 \mathrm{~B}, 38 \mathrm{C}$ & $\begin{array}{l}\text { sediment } \\
\text { sampler }\end{array}$ \\
\hline 39 & V-BP/Fe-Id & Biological Phosphate/Fe(0) & Vertical & $39 a, 39 b, 39 c$ & $\begin{array}{l}\text { sediment } \\
\text { profiler }\end{array}$ \\
\hline 40 & H-Fe-IIId & $\mathrm{Fe}(0)$ & Horizontal & $40 \mathrm{~A}, 40 \mathrm{~B}, 40 \mathrm{C}$ & $\begin{array}{l}\text { sediment } \\
\text { sampler }\end{array}$ \\
\hline
\end{tabular}

*d- duplicate

**A, B $, \mathrm{C}, \ldots .$. - the uppercase letters stand for split samples in the horizontal treatments; $1 \mathrm{~cm}$ slices or if possible $0.5 \mathrm{~cm}$ slices $* * * \mathrm{a}, \mathrm{b}, \mathrm{c}, \mathrm{d}, \mathrm{e}, \mathrm{f}, \mathrm{g}, \mathrm{h}, \ldots$. the lowercase letters stand for split samples in the vertical treatments; $1 \mathrm{~cm}$ layers 


\section{Total Digestion of the sediment samples from the TNX OD}

A total digestion of the sediment from the TNX OD from vertical and horizontal treatments was performed on 0.6-gram samples with $14.5 \mathrm{ml} \mathrm{HNO}_{3}, 4 \mathrm{ml} \mathrm{H}_{2} \mathrm{SO}_{4}$, and 2 $\mathrm{ml}$ of $\mathrm{HCl}$. All analyses were performed on Star 6 Plus System. The final volume of each sample was $100 \mathrm{ml}$. Samples were filtered (\#41). The extracts were sent to WSRC, EPD, EMS Lab (735 B) for ICP -AES analysis. The samples were analyzed by Alex Guanlao (phone: 803-725-2432). SC DHEC Certification: 02550001 
WSRC-TR-2003-00579, REV. 0

Table 6C. Total digestion of the sediment samples from the TNX OD; vertical and horizontal treatments.

\begin{tabular}{|c|c|c|c|c|c|c|c|c|c|c|}
\hline Lab \# & Sample ID & Treatment & $\begin{array}{l}\text { Sample } \\
\text { wt } \\
{[\mathrm{g}]} \\
\end{array}$ & $\begin{array}{l}\text { Final } \\
\text { vol. } \\
\text { of } \\
\text { solution } \\
{[\mathrm{ml}]} \\
\end{array}$ & $\begin{array}{l}\text { Correction } \\
\text { Factor }\end{array}$ & $\begin{array}{l}\text { Vessel } \\
\text { ID }\end{array}$ & $\begin{array}{l}\text { Raw } \\
\text { data } \\
\mathrm{P} \\
\mathrm{mg} / \mathrm{kg} \\
\end{array}$ & $\begin{array}{l}\begin{array}{l}\text { Final } \\
\text { conc. }\end{array} \\
\mathrm{P} \\
\mathrm{Mg} / \mathrm{kg} \\
\end{array}$ & $\begin{array}{l}\begin{array}{l}\text { Raw } \\
\text { data }\end{array} \\
\mathrm{Fe} \\
\mathrm{mg} / \mathrm{kg} \\
\end{array}$ & $\begin{array}{l}\text { Final } \\
\text { conc. } \\
\mathrm{Fe} \\
\mathrm{mg} / \mathrm{kg} \\
\end{array}$ \\
\hline & & & & Vertic & al Treatments & & & & & \\
\hline 1 & $\begin{array}{l}\text { blank } 1 \\
\text { blank } 2\end{array}$ & & & & & 23 & $\begin{array}{l}0.527 \\
1.964\end{array}$ & & $\begin{array}{r}0.3444 \\
0.05689\end{array}$ & \\
\hline 3 & V-control-IIb & Vertical - Control & 0.6010 & 100 & 166.4 & 18 & 3.779 & 628.8 & 43.39 & 7220 \\
\hline 4 & V-control-IIc & Vertical - Control & 0.6080 & 100 & 164.5 & 12 & 3.678 & 604.9 & 44.53 & 7324 \\
\hline 5 & V-control-Ia & Vertical - Control & 0.6059 & 100 & 165.0 & 2 & 2.502 & 412.9 & 38.86 & 6414 \\
\hline 6 & V-control-Ib & Vertical - Control & 0.6015 & 100 & 166.3 & 6 & 2.826 & 469.8 & 37.14 & 6175 \\
\hline 7 & V-control-Ic & Vertical - Control & 0.6071 & 100 & 164.7 & 21 & 2.648 & 436.2 & 40.1 & 6605 \\
\hline 8 & V-NCA-Ia & Vertical - NCAI & 0.5955 & 100 & 167.9 & 4 & 12.09 & 2030.2 & 37.1 & 6230 \\
\hline 13 & V-NCA-IIc & Vertical - NCAII & 0.5994 & 100 & 166.8 & 4 & 2.368 & 395.1 & 23.04 & 3844 \\
\hline 14 & V-BA-Ia & Vertical - BAI & 0.596 & 100 & 167.8 & 23 & 20.58 & 3453.0 & 45.61 & 7653 \\
\hline 15 & V-BA-Ib & Vertical - BAI & 0.5988 & 100 & 167.0 & 17 & 15.52 & 2591.9 & 43.36 & 7241 \\
\hline 16 & V-BA-Ic & Vertical - BAI & 0.6087 & 100 & 164.3 & 2 & 9.254 & 1520.3 & 32.43 & 5328 \\
\hline 17 & V-BA-IIa & Vertical - BAII & 0.5962 & 100 & 167.7 & 61 & 14.75 & 2474.0 & 35.93 & 6027 \\
\hline 18 & V-BA-IIb & Vertical - BAII & 0.6069 & 100 & 164.8 & 21 & 6.972 & 1148.8 & 35.7 & 5882 \\
\hline 19 & V-BA-IIc & Vertical - BAII & 0.5924 & 100 & 168.8 & $18^{\prime}$ & 3.428 & 578.7 & 39.97 & 6747 \\
\hline 20 & V-Fe-Ia & Vertical - FeI & 0.6043 & 100 & 165.5 & 8 & 3.407 & 563.8 & 58.23 & 9636 \\
\hline 21 & V-Fe-Ib & Vertical - FeI & 0.6067 & 100 & 164.8 & 9 & 3.453 & 569.1 & 35.09 & 5784 \\
\hline
\end{tabular}


WSRC-TR-2003-00579, REV. 0

\begin{tabular}{|c|c|c|c|c|c|c|c|c|c|c|}
\hline 22 & V-Fe-Ic & Vertical - FeI & 0.6084 & 100 & 164.4 & 14 & 3.385 & 556.4 & 33.48 & 5503 \\
\hline 23 & V-Fe-IIa & Vertical - FeII & 0.6066 & 100 & 164.9 & 12 & 3.934 & 648.5 & 104.4 & 17211 \\
\hline 24 & V-Fe-IIb & Vertical - FeII & 0.5997 & 100 & 166.8 & 5 & 4.133 & 689.2 & 66.54 & 11096 \\
\hline 25 & V-Fe-IIc & Vertical - FeII & 0.5997 & 100 & 166.8 & 6 & 3.863 & 644.2 & 64.67 & 10784 \\
\hline \multicolumn{11}{|c|}{ Horizontal Treatments } \\
\hline 1 & $\mathrm{H}-\mathrm{BA} / \mathrm{Fe}-\mathrm{IA}$ & $\begin{array}{l}\text { Horizontal - } \\
\text { BA/FeI } \\
\text { Horizontal - }\end{array}$ & 0.6055 & 100 & 165.2 & 2 & 5.237 & 864.9 & 57.15 & 9438 \\
\hline 2 & $\mathrm{H}-\mathrm{BA} / \mathrm{Fe}-\mathrm{IB}$ & $\begin{array}{l}\text { BA/FeI } \\
\text { Horizontal - }\end{array}$ & 0.5904 & 100 & 169.4 & 14 & 4.141 & 701.4 & 38.09 & 6452 \\
\hline 3 & $\mathrm{H}-\mathrm{BA} / \mathrm{Fe}-\mathrm{IC}$ & $\begin{array}{l}\mathrm{BA} / \mathrm{FeI} \\
\text { Horizontal - }\end{array}$ & 0.597 & 100 & 167.5 & 5 & 4.084 & 684.1 & 41.26 & 6911 \\
\hline 4 & H-BA/Fe-ID & $\begin{array}{l}\mathrm{BA} / \mathrm{FeI} \\
\text { Horizontal - }\end{array}$ & 0.6068 & 100 & 164.8 & 61 & 3.224 & 531.3 & 32.81 & 5407 \\
\hline 5 & $\mathrm{H}-\mathrm{BA} / \mathrm{Fe}-\mathrm{IE}$ & $\begin{array}{l}\mathrm{BA} / \mathrm{FeI} \\
\text { Horizontal - }\end{array}$ & 0.6093 & 100 & 164.1 & 12 & 2.898 & 475.6 & 28.74 & 4717 \\
\hline 6 & $\mathrm{H}-\mathrm{NCA} / \mathrm{Fe}-\mathrm{IA}$ & $\begin{array}{l}\mathrm{NCA} / \mathrm{FeI} \\
\text { Horizontal - }\end{array}$ & 0.592 & 100 & 168.9 & 21 & 9.943 & 1679.6 & 70.09 & 11840 \\
\hline 7 & H-NCA/Fe-IB & $\begin{array}{l}\mathrm{NCA} / \mathrm{FeI} \\
\text { Horizontal - }\end{array}$ & 0.5932 & 100 & 168.6 & 8 & 5.324 & 897.5 & 59.68 & 10061 \\
\hline & H-NCA/Fe-IC & $\begin{array}{l}\mathrm{NCA} / \mathrm{FeI} \\
\text { Horizontal - }\end{array}$ & 0.6028 & 100 & 165.9 & 8 & 5.585 & 926.5 & 61.83 & 10257 \\
\hline & H-NCA/Fe-ID & $\begin{array}{l}\mathrm{NCA} / \mathrm{FeI} \\
\text { Horizontal - }\end{array}$ & 0.608 & 100 & 164.5 & 23 & 4.846 & 797.0 & 70.9 & 11661 \\
\hline & H-NCA/Fe-IE & NCA/FeI & 0.6044 & 100 & 165.5 & 9 & 4.333 & 716.9 & 62.15 & 10283 \\
\hline & H-BA-IA & Horizontal - BAI & 0.5933 & 100 & 168.5 & 5 & 41.71 & 7030.2 & 57.04 & 9614 \\
\hline & H-BA-IB & Horizontal - BAI & 0.5992 & 100 & 166.9 & 12 & 8.794 & 1467.6 & 48.26 & 8054 \\
\hline & H-BA-IC & Horizontal - BAI & 0.6048 & 100 & 165.3 & 2 & 6.916 & 1143.5 & 31.78 & 5255 \\
\hline & H-BA-ID & Horizontal - BAI & 0.6093 & 100 & 164.1 & 17 & 2.593 & 425.6 & 22.09 & 3625 \\
\hline & H-BA-IE & Horizontal - BAI & 0.6039 & 100 & 165.6 & 8 & 2.217 & 367.1 & 25.29 & 4188 \\
\hline & H-NCA-IA & Horizontal - NCAI & 0.6032 & 100 & 165.8 & 61 & 46.05 & 7634.3 & 43.79 & 7260 \\
\hline & H-NCA-IB & Horizontal - NCAI & 0.6029 & 100 & 165.9 & 23 & 4.616 & 765.6 & 54.56 & 9050 \\
\hline & H-NCA-IC & Horizontal - NCAI & 0.5963 & 100 & 167.7 & 21 & 4.393 & 736.7 & 37.62 & 6309 \\
\hline & H-NCA-ID & Horizontal - NCAI & 0.5923 & 100 & 168.8 & 6 & 5.11 & 862.7 & 52.02 & 8783 \\
\hline
\end{tabular}


WSRC-TR-2003-00579, REV. 0

\begin{tabular}{|c|c|c|c|c|c|c|c|c|c|c|}
\hline H-NCA-IE & Horizontal - NCAI & 0.6091 & 100 & 164.2 & & 18 & 3.555 & 583.6 & 37.71 & 6191 \\
\hline H-Fe-IA & Horizontal - $\mathrm{Fe}(0) \mathrm{I}$ & 0.6084 & 100 & 164.4 & & 14 & 7.494 & 1231.8 & 213.9 & 35158 \\
\hline H-Fe-IB & Horizontal - $\mathrm{Fe}(0) \mathrm{I}$ & 0.5924 & 100 & 168.8 & & 9 & 6.922 & 1168.5 & 125.3 & 21151 \\
\hline H-Fe-IC & Horizontal - $\mathrm{Fe}(0) \mathrm{I}$ & 0.6049 & 100 & 165.3 & & 6 & 6.416 & 1060.7 & 107.2 & 17722 \\
\hline $\mathrm{H}-\mathrm{Fe}-\mathrm{ID}$ & Horizontal - $\mathrm{Fe}(0) \mathrm{I}$ & 0.6099 & 100 & 164.0 & & 17 & 5.568 & 912.9 & 91.68 & 15032 \\
\hline $\mathrm{H}-\mathrm{Fe}-\mathrm{IE}$ & $\begin{array}{l}\text { Horizontal - } \mathrm{Fe}(0) \mathrm{I} \\
\text { Horizontal - }\end{array}$ & 0.6098 & 100 & 164.0 & & 21 & 5.9 & 967.5 & 76.01 & 12465 \\
\hline H-Control-IIA & $\begin{array}{l}\text { Control II } \\
\text { Horizontal - }\end{array}$ & 0.5983 & 100 & 167.1 & & 5 & 5.274 & 881.5 & 50 & 8357 \\
\hline H-Control-IIB & $\begin{array}{l}\text { Control II } \\
\text { Horizontal - }\end{array}$ & 0.599 & 100 & 166.9 & & 2 & 6.689 & 1116.7 & 67.12 & 11205 \\
\hline H-Control-IIC & $\begin{array}{l}\text { Control II } \\
\text { Horizontal - }\end{array}$ & 0.5991 & 100 & 166.9 & & 14 & 6.855 & 1144.2 & 72.31 & 12070 \\
\hline H-Control-IID & $\begin{array}{l}\text { Control II } \\
\text { Horizontal - }\end{array}$ & 0.601 & 100 & 166.4 & & 4 & 4.966 & 826.3 & 82.1 & 13661 \\
\hline $\begin{array}{l}\text { H-Control-IIE } \\
\text { Blank I }\end{array}$ & Control II & 0.6058 & 100 & 165.1 & $18 "$ & 18 & $\begin{array}{l}4.467 \\
1.817\end{array}$ & 737.4 & $\begin{array}{r}77.74 \\
0.04491\end{array}$ & 12833 \\
\hline Blank II & & & & & & 18 & 1.869 & & 0.03024 & \\
\hline
\end{tabular}


Table 7C. The results from a total digestion of the sediment samples from the TNX OD; vertical treatments.

\begin{tabular}{lrrrr}
\hline $\begin{array}{l}\text { Vertical } \\
\text { Treatments }\end{array}$ & $\begin{array}{c}\text { P - AVG } \\
\mathrm{mg} / \mathrm{kg}\end{array}$ & $\mathrm{P}$ - STDEV & $\begin{array}{c}\text { Fe - AVG } \\
\mathrm{Mg} / \mathrm{kg}\end{array}$ & Fe - STDEV \\
\hline \hline V-control-a & 451 & 54 & 7105 & 977 \\
V-control-b & 549 & 112 & 6697 & 739 \\
V-control-c & 521 & 119 & 6965 & 508 \\
V-NCA-a & 1222 & 1144 & 5301 & 1314 \\
V-NCA-b & 940 & 665 & 4542 & 1930 \\
V-NCA-c & 578 & 200 & 4863 & 1442 \\
V-BP-a & 1289 & 1048 & 6840 & 1150 \\
V-BP-b & 933 & 675 & 6562 & 961 \\
V-BP-c & 557 & 229 & 6037 & 1004 \\
V-Fe-a & 2001 & 2054 & 13423 & 5356 \\
V-Fe-b & 1524 & 1510 & 8440 & 3756 \\
V-Fe-c & 958 & 796 & 8143 & 3734 \\
P-background & 561 & 86 & 6101 & 1272 \\
\hline V - vertical, BP - Biological Phosphate, NCA - North Carolina Apatite, Fe - Fe(0) \\
a, b, c - distance from P/Fe source (amendment) in cm: a- 0 to 1cm, b- 1 to 2cm, and c - \\
2 to 3 cm
\end{tabular}


Table 8C. The results from a total digestion of the sediment samples from the TNX OD; horizontal treatments.

\begin{tabular}{|c|c|c|c|c|c|}
\hline \multirow[t]{2}{*}{ Treatments } & \multicolumn{3}{|c|}{ Concentration of $\mathrm{P}$} & \multirow[b]{2}{*}{$\mathrm{mg} / \mathrm{kg}$} & \multirow[b]{2}{*}{$\mathrm{mg} / \mathrm{kg}$} \\
\hline & $\mathrm{Mg} / \mathrm{kg}$ & $\begin{array}{l}\mathrm{Mg} / \mathrm{kg} \\
\text { Distance }\end{array}$ & $\mathrm{mg} / \mathrm{kg}$ & & \\
\hline & $0-1 \mathrm{~cm}$ & $1-2 \mathrm{~cm}$ & $2-3 \mathrm{~cm}$ & $3-4 \mathrm{~cm}$ & $4-5 \mathrm{~cm}$ \\
\hline H-control & 881 & 1117 & 11144 & 826 & 737 \\
\hline $\mathrm{H}-\mathrm{Fe}$ & 1232 & 1168 & 1061 & 913 & 968 \\
\hline P-background & 1057 & 1143 & 1102 & 870 & 852 \\
\hline H-BP & 7030 & 1468 & 1144 & 426 & 367 \\
\hline $\mathrm{H}-\mathrm{NCA}$ & 7634 & 766 & 737 & 863 & 584 \\
\hline $\mathrm{H}-\mathrm{BP} / \mathrm{Fe}$ & 865 & 701 & 684 & 531 & 476 \\
\hline \multirow[t]{2}{*}{$\mathrm{H}-\mathrm{NCA} / \mathrm{Fe}$} & 1680 & 898 & 927 & 797 & 717 \\
\hline & $\begin{array}{c}\mathrm{Fe} \\
\mathrm{Mg} / \mathrm{kg} \\
8357\end{array}$ & $\begin{array}{c}\mathrm{Fe} \\
\mathrm{Mg} / \mathrm{kg} \\
11205\end{array}$ & $\begin{array}{c}\mathrm{Fe} \\
\mathrm{mg} / \mathrm{kg} \\
12070\end{array}$ & $\begin{array}{c}\mathrm{Fe} \\
\mathrm{mg} / \mathrm{kg} \\
13661\end{array}$ & $\begin{array}{c}\mathrm{Fe} \\
\mathrm{mg} / \mathrm{kg} \\
12833\end{array}$ \\
\hline$\overline{\mathrm{H} \text { H-BP }}$ & 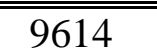 & 8054 & $\overline{c 5255}$ & 3625 & 年188 \\
\hline $\mathrm{H}-\mathrm{NCA}$ & 7260 & 9050 & 6309 & 8783 & 6191 \\
\hline $\begin{array}{l}\text { Fe- } \\
\text { background }\end{array}$ & 8410 & 9436 & 7878 & 8690 & 7737 \\
\hline (stdev) & 1178 & 1611 & 3668 & 5018 & 4525 \\
\hline $\mathrm{H}-\mathrm{Fe}$ & 35158 & 21151 & 17722 & 15032 & 12465 \\
\hline $\mathrm{H}-\mathrm{BP} / \mathrm{Fe}$ & 9438 & 6452 & 6911 & 5407 & 4717 \\
\hline $\mathrm{H}-\mathrm{NCA} / \mathrm{Fe}$ & 11840 & 10061 & 10257 & 11661 & 10283 \\
\hline \multicolumn{6}{|c|}{$\begin{array}{l}\text { H- horizontal, BP - Biological Phosphate, NCA - North Carolina Apatite, Fe - } \\
\mathrm{Fe}(0) \\
\text { Distance from P/Fe source (amendment) in cm }\end{array}$} \\
\hline
\end{tabular}

